\title{
Building a tuberculosis-free world: The Lancet Commission on tuberculosis
}

\author{
Michael J A Reid*, Nimalan Arinaminpathy*, Amy Bloom*, Barry R Bloom*, Catharina Boehme*, Richard Chaisson*, Daniel P Chin*, \\ Gavin Churchyard*, Helen Cox*, Lucica Ditiu*, Mark Dybul*, Jeremy Farrar*, Anthony S Fauci*, Endalkachew Fekadu*, Paula I Fujiwara*, \\ Timothy B Hallett*, Christy L Hanson*, Mark Harrington*, Nick Herbert*, Philip C Hopewell*, Chieko Ikeda*, Dean T Jamison*t, AamirJ Khan*, \\ Irene Koek*, Nalini Krishnan*, Aaron Motsoaledi*, Madhukar Pai*, Mario C Raviglione*, Almaz Sharman*, Peter M Small*, \\ Soumya Swaminathan*t, Zelalem Temesgen*, Anna Vassall*, Nandita Venkatesan*, Kitty van Weezenbeek*, Gavin Yamey*, Bruce D Agins, \\ Sofia Alexandru, Jason R Andrews, Naomi Beyeler, Stela Bivol, Grania Brigden, Adithya Cattamanchi, Danielle Cazabon, Valeriu Crudu, \\ Amrita Daftary, Puneet Dewan, Laurie K Doepel, Robert W Eisinger, Victoria Fan, Sara Fewer, Jennifer Furin, Jeremy D Goldhaber-Fiebert, \\ Gabriela B Gomez, Stephen M Graham, Devesh Gupta, Maureen Kamene, Sunil Khaparde, Eunice W Mailu, Enos O Masini, Lorrie McHugh, \\ Ellen Mitchell, Suerie Moon, Michael Osberg, Tripti Pande, Lea Prince, Kirankumar Rade, Raghuram Rao, Michelle Remme, James A Seddon, \\ Casey Selwyn, Priya Shete, Kuldeep S Sachdeva, Guy Stallworthy, Juan F Vesga, Valentina Vilc, Eric P Goosby*
}

\section{Executive summary}

Tuberculosis can be treated, prevented, and cured. Rapid, sustained declines in tuberculosis deaths in many countries during the past 50 years provide compelling evidence that ending the pandemic is feasible. Yet this disease-which has plagued humanity since before recorded history and has killed hundreds of millions of people over the past two centuries-remains a relentless scourge. In 2017, 1.6 million people died from tuberculosis, including 300000 people with HIV, representing more deaths than any other infectious disease. Moreover, in many parts of the world, drugresistant forms of tuberculosis threaten struggling control efforts. The world can no longer ignore the enormous pall cast by the tuberculosis epidemic. Going forward, the global tuberculosis response must be an inclusive, comprehensive response within the broader sustainable development agenda. No one-size-fits-all approach can succeed.

In September, 2018, the first-ever UN High-Level Meeting (UNHLM) on tuberculosis resolved to make ending this disease a global priority. Heads of State and government representatives from all UN member states committed to take major steps towards building a tuberculosis-free world, including ambitious goals to treat successfully 40 million people with tuberculosis and to prevent at least 30 million becoming ill between 2018 and 2022, through the provision of tuberculosis preventive treatment.

Achieving these objectives will not be easy. First, many people with tuberculosis, especially the poorest, can neither access nor afford high-quality tuberculosis services. Diagnostic and treatment capacity is not always located where the need is greatest. Consequently, up to $35 \%$ of people with tuberculosis disease are not being diagnosed and treated, or made known to national tuberculosis programmes.

Second, strategies to identify people with active disease in high-risk populations, such as people with HIV, household contacts, migrants, and prisoners, are at best implemented in a piecemeal manner. Furthermore, despite compelling evidence that tuberculosis preventive therapy is life-saving for some among these populations, it is often not offered in high-burden countries.

Third, tuberculosis research and development is chronically underfunded. Unless urgent steps are taken to substantially increase research and development funding to enable the development of new and more patientfriendly treatment strategies, as well as transformative diagnostics and vaccines, rapid declines in tuberculosis mortality will prove difficult.

Finally, global efforts to end tuberculosis have been undermined by insufficient political will and financial investments. Economic analysis commissioned for this report show that the value of the benefits of averting a death from tuberculosis exceeds the value of its costs by more than a factor of 3 to 5 , and is likely to be considerably more in many settings. Available funding for tuberculosis programmes efforts fall considerably short of what is required.

Working under the assumption that with smart investments based on sound science, accelerated research and development, and a shared responsibility, we can end tuberculosis within a generation, this Commission set out to answer the question of how tuberculosis highburden countries and their development partners should target their future investments to ensure that ending tuberculosis is achieved.

The Commission asserts that to realise the Sustainable Development Goal of reducing tuberculosis mortality by $90 \%$ from 2015, as proposed in the WHO's End TB [tuberculosis] Strategy, and to achieve a tuberculosis-free world within a generation, tuberculosis investments must be focused on the five priority areas (see Key messages panel).

\section{Seizing this moment}

Although the challenges of ending tuberculosis are many, the outlook is encouraging. We have rapid, sensitive diagnostic tools, and the promise of potent tuberculosis treatment strategies in the pipeline. Programmatic innovations, new health technologies, digital solutions, sustained global economic growth, increased commitment to achieve universal health coverage (UHC), and

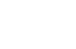


(V Fan ScD), Harvard University, Cambridge, MA, USA; Foundation for Innovative New Diagnostics, Geneva, Switzerland (C Boehme MD); Departments of Medicine, Epidemiology, and International Health, Johns Hopkins School of Medicine, Baltimore, MA, USA (Prof R Chaisson MD); Bill \& Melinda Gates Foundation, Seattle, WA, USA (D P Chin MD, CL Hanson PhD, ( Selwyn MPhil); The Aurum Institute, Johannesburg, South Africa (G Churchyard PhD); Department of Pathology, Institute of Infectious Disease and Molecular Medicine, University of Cape Town, Cape Town, South Africa (H Cox PhD); Stop TB Partnership (L Ditiu MD), WHO, Geneva, Switzerland (S Swaminathan MD);

Department of Medicine, Centre for Global Health and Quality, Georgetown University, Washington, DC,

USA (Prof M Dybul MD); The Wellcome Trust, London, UK (Prof J Farrar PhD); National Institute of Allergy and Infectious Diseases, US

National Institutes of Health, Maryland, MA, USA (A S Fauci MD,

L K Doepel, R W Eisinger PhD); Volunteer Health Services, Addis Ababa, Ethiopia

(E Fekadu BPharm); Department of Tuberculosis and HIV The International Union Against Tuberculosis and Lung Disease, Paris, France (G Brigden MD, P I Fujiwara MD, SM Graham PhD); Treatment Action Group, New York, NY, USA (M Harrington); Global TB Caucus, Houses of Parliament,

London, UK (N Herbert CBE); Department of GLobal Health, Ministry of Heath, Labor and Welfare, Tokyo, Japan (C Ikeda PhD); Interactive Research \& Development, Karachi, Pakistan (A) Khan MBBS); Resource Group for Education and Advocacy for Community Health, Chennai, India (N Krishnan MD); South African National Department of Health, Pretoria, South Africa (A Motsoaledi MD); Department of Epidemiology, Biostatistics and Occupational Health (Prof M Pai MD, A Daftary PhD), and McGill International

\section{Key messages}

The Commission recommends five priority investments to achieve a tuberculosis-free world within a generation. These investments are designed to fulfil the mandate of the UN High Level Meeting on tuberculosis. In addition, they answer the question of how countries with high-burden tuberculosis and their development partners should target their future investments to ensure that ending tuberculosis is achievable.

Invest first to ensure that high quality rapid diagnostics and treatment are provided to all individuals receiving care for tuberculosis, wherever they seek care

This priority includes rapid drug susceptibility testing and second-line treatment for resistant forms of tuberculosis. Achieving universal, high-quality person-centred and family-centred care-including sustained improvement in the performance of private sector providers-usually should be the top policy and budget priority.

Reach people and populations at high risk for tuberculosis (such as household and other close contacts of people with tuberculosis, and people with HIV) and bring them into care Active case-finding and treatment in high-risk populations demands adequate resources to reach and care for these populations. At the same time, reaching certain high-risk populations, such as people co-infected with tuberculosis and HIV, for tuberculosis preventive therapy is essential to achieve epidemiologic control. Once high-risk populations have access to affordable, high-quality diagnostic, treatment and preventive services, invest in identifying tuberculosis cases in the general population, primarily by strengthening the capacity to deliver health services and move toward universal health coverage.

Increase investment to accelerate tuberculosis research and development and bring new diagnostics, therapeutic strategies, and vaccines to clinical practice to quickly end the pandemic

Strong advocacy with science ministries and research-oriented pharmaceutical companies is crucial, including ministries and

growing political momentum to definitively address tuberculosis, could all make ending the pandemic within a generation more feasible than ever before.

\section{Moving forward with bold, comprehensive strategies}

Globally, the priority must be to deliver person-centred and family-centred services to all individuals with tuberculosis who present to care. This approach means ensuring that high-quality diagnostics, treatment, and prevention modalities are available to all, wherever they seek care. Improving quality of tuberculosis care in the private sector is crucial to end tuberculosis in high incidence countries such as India, the country with the highest tuberculosis burden. Modelling shows that optimising private sector engagement in India could avert 8 million deaths from tuberculosis between 2019 and 2045 (appendix p 3). In high drug-resistant tuberculosis burden countries, access companies in middle-income countries, to highlight the importance of investing in new tools. Financing the early uptake of new products will provide important confidence signals to product developers.

Make investment in tuberculosis programmes a shared responsibility, increasing development assistance for tuberculosis according to the financial needs of individual low-income and middle-income countries

As countries successfully mobilise more domestic resources towards tuberculosis programmes, external assistance to middle-income countries should address the following priorities: reduce the spread of drug-resistant tuberculosis in all affected low-income and middle-income countries; facilitate market-shaping activities to enable access to high quality drugs and diagnostics for high-burden countries; and finance tuberculosis research and development, including product development as well as population, policy, and implementation research that will provide lessons and international sharing of best practices.

Hold countries and key stakeholders accountable for progress made towards ending tuberculosis

Accountability entails establishing independent, multisectoral processes, such as national tuberculosis report cards, to ensure that all stakeholders carry out their responsibilities to contribute to ending the pandemic. Accountability mechanisms should not only assess progress, but also guarantee that Heads of Governments, national tuberculosis programmes, and even regional and site-level clinics, as well as key non-governmental organisations, take the necessary corrective actions to remove obstacles to ending tuberculosis.

to rapid drug susceptibility testing (DST) and second-line drugs is essential to success. In Moldova, where more than $25 \%$ of all tuberculosis cases are drug-resistant, improving access to DST and second-line drugs would reduce mortality from drug-resistant tuberculosis by $44 \%$ in the coming generation (appendix p 3).

Secondly, tuberculosis programme budgets must increase to enable reaching these people and populations at high risk of tuberculosis. In Kenya, for example, where the proportions of HIV and tuberculosis coinfection are high, scaling up access to both antiretroviral therapy and tuberculosis preventive therapy can help save an additional 3 million lives over the next generation (appendix p 3).

However, ultimately, the fight against tuberculosis will not be won unless countries also ensure that everyone, not just high-risk groups, can access essential health 
services without risking catastrophic medical costs. Achieving UHC is crucial to sustain an end to tuberculosis.

\section{Invest more to accelerate tuberculosis research and development}

Although we need to intensify efforts by rapidly scalingup proven interventions, ending the tuberculosis epidemic in high-burden countries also will require new and improved tools, and effective adoption of enabling technologies and programmatic innovation. In the near term (5-10 years), increasing the investment in diagnostic, therapeutic, and prevention research and development, as well as population, policy, and implementation research to rapidly transform research findings for use in tuberculosis programmes, can yield significant returns. A longer-term (10-15 years) goal must be development of an effective vaccine as the surest means of ending the pandemic.

Reaching these research goals will require substantially increasing global investment in tuberculosis research and development, from US\$772 million per year in 2017 to at least US\$2 billion per year during the next 4 years to develop the essential tuberculosis tools and move them from the pipeline into production. This investment must be weighed against the cost of inaction: in India, for example, even with optimal implementation of all existing tools, unavoidable tuberculosis deaths will cost the economy at least US\$32 billion each year over the next 30 years. Although greater investment from high-income countries is imperative, high-burden middle-income countries, such as Brazil, China, India, Russia, and South Africa, can transform the global tuberculosis research and development agenda through increased financial investment, collaborative networks, and incentivised interdisciplinary research partnerships.

\section{Sustained financing for tuberculosis programmes is crucial}

Everyone dedicated to achieving an end to tuberculosisincluding affected countries, donor agencies, the private sector, and foundations-must redouble their efforts to finance strategies that we know work now and, more importantly, to support novel strategies that will cause a substantial decline in the trajectory of the pandemic in the future. High-burden countries must substantially increase financial resources to fight tuberculosis. Countries like Bangladesh, China, Indonesia, and Zambia can increase their annual tuberculosis expenditures more than five-fold over the next 5 years, through increased revenue generation and allocation of greater budgetary resources to health. The dividend of this investment can be substantial: recent history shows that those countries that have achieved extraordinary progress against tuberculosis have reaped broad economic and health benefits that continue to this day.

\section{Accountability and shared responsibility to track progress}

To ensure that the results required to end tuberculosis occur within a generation, we must employ clear accountability mechanisms. These mechanisms can ensure that corrective actions are taken as appropriate, and that the financial, political, and programmatic barriers to deliver comprehensive tuberculosis care are removed. Tuberculosis report cards, or similar independent review processes, for heads of government, donor agencies, and key non-governmental stakeholders can help ensure that financial commitments and other promises are kept, and that progress milestones are met. Accountability to ensure multisectoral action to address risk factors for tuberculosis, such as air pollution, tobacco use, diabetes, and undernutrition, is also important. This Commission proposes the establishment of a Tuberculosis Observatory to evaluate progress made by countries in meeting the targets outlined in the UNHLM declaration, and to determine whether the recommendations in this Commission are a catalysing programme and policy changes.

Creating an enabling environment to eliminate tuberculosis within a country also requires engaging civil society and acknowledging their crucial role in all aspects of tuberculosis programming. We must strengthen civil society's involvement by increasing their decisionmaking contributions to planning, implementation, and accountability. Additionally, we must uphold and defend the rights of all people with tuberculosis and those most at risk, and put in place policies and practices to protect them against stigmatisation and discrimination.

This Commission cites grounds for optimism: ending tuberculosis is feasible by rapidly strengthening and expanding our health delivery systems to effectively implement proven interventions we know work; accelerating innovative science to develop and implement new and improved approaches to diagnose, treat, and prevent drugsensitive and drug-resistant tuberculosis; and substantially increasing the political will to catalyse sustainable financing for tuberculosis. There is no room for complacency; clear accountability is necessary to ensure that promises are kept and targets reached. We must act quickly and strategically to save the next generation from this preventable and curable disease.

\section{Introduction} 'Knowing is not enough; we must apply. Willing is not
enough; we must do."

Goethe

Progress against tuberculosis: moving forward, but not fast enough

In 1993, WHO declared tuberculosis a public health emergency. ${ }^{1}$ WHO urged governments worldwide to substantially scale up their efforts to control tuberculosis and within 1 year unveiled the so-called directly observed
TB Center (M Pai MD,

A Daftary PhD

D Cazabon MScPh,

TPande MScPH), McGill

University, Montreal, QC

Canada; University of Milan,

Milan, Italy

(Prof M C Raviglione MD); Global Studies Institute, University of Geneva, Geneva, Switzerland (Prof M C Raviglione); Academy of Preventive Medicine of Kazakhstan, Almaty, Kazakhstan

(Prof A Sharman MD); Global Health Institute, School of Medicine, Stony Brook University, Stony Brook, NY, USA (Prof P M Small MD); Department of Infectious Diseases, Mayo Clinic, Rochester, MI, USA (Prof Z Temesgen MD); Department of Global Health and Development, Faculty of Public Health and Policy,

London School of Hygiene and Tropical Medicine, London, UK (Prof A Vassall PhD,

G B Gomez PhD); Amsterdam Institute for Global Health and Development, University of Amsterdam, Amsterdam, Netherlands (Prof A Vassal); The Economic Times, Mumbai, India (N Venkatesan MSc); KNCV Tuberculosis Foundation, The Haag, Netherlands (K van Weezenbeek MD); Center for Policy Impact in Global Health, Duke Global Health Institute, Duke University, Durham, NC, USA (Prof G Yamey MD); Institutul de Ftiziopneumologie Chiril Draganiuc, Chisinau, Moldova (S Alexandru MD); Division of Infectious Diseases and Geographic Medicine (J R Andrews MD), and Centers for Health Policy and Primary Care and Outcomes Research (J D Goldhaber-Fiebert PhD, L Prince PhD), Stanford University, Stanford, CA, USA; Center for Health Policies and Studies, Chisinau, Moldova (S Bivol, MD, V Crudu MD); Bill \& Melinda Gates Foundation, New Delhi, India (P Dewan MD); Division of Infectious Diseases \& HIV Medicine, Case Western Reserve University, Cleveland, $\mathrm{OH}$, USA (J Furin MD); Central Tuberculosis Division, WHO, New Delhi, India (K Rade MD); General TB control (S Khaparde MD), Revised National TB Control Program (D Gupta MD, K S Sachdeva MBA), 


\begin{tabular}{|c|c|c|c|c|c|c|c|c|}
\hline & $\begin{array}{l}\text { Deaths } \\
\text { (thousands) in } \\
2000\end{array}$ & $\begin{array}{l}\text { Deaths } \\
\text { (thousands) in } \\
2017^{*}\end{array}$ & $\begin{array}{l}\text { Cumulative } \\
\text { percentage deaths } \\
\text { in } 2017 \text { (\% of total } \\
\text { tuberculosis } \\
\text { deaths) }\end{array}$ & $\begin{array}{l}\text { Death rate in } \\
2000(\%) \dagger\end{array}$ & $\begin{array}{l}\text { Death rate in } \\
2017(\%) \dagger\end{array}$ & $\begin{array}{l}\text { Rate of decline in } \\
\text { deaths from } \\
2000-17 \ddagger\end{array}$ & $\begin{array}{l}\text { Rate of decline in } \\
\text { death rates from } \\
2000-17 \ddagger\end{array}$ & $\begin{array}{l}\text { Demographic } \\
\text { headwinds } \$\end{array}$ \\
\hline World & 2340 & 1570 & $100 \%$ & 38 & 21 & $2 \cdot 3 \%$ & $3 \cdot 5 \%$ & $1 \cdot 1 \%$ \\
\hline India & 717 & 421 & $27 \%$ & 68 & 31 & $3 \cdot 1 \%$ & $4.6 \%$ & $1.5 \%$ \\
\hline Nigeria & 128 & 155 & $37 \%$ & 105 & 81 & $-1 \cdot 1 \%$ & $1 \cdot 5 \%$ & $2.7 \%$ \\
\hline Indonesia & 154 & 116 & $44 \%$ & 73 & 44 & $1.7 \%$ & $3.0 \%$ & $1 \cdot 3 \%$ \\
\hline South Africa & 86 & 78 & $49 \%$ & 187 & 138 & $0.6 \%$ & $1.8 \%$ & $1 \cdot 2 \%$ \\
\hline Bangladesh & 95 & 60 & $53 \%$ & 72 & 36 & $2 \cdot 7 \%$ & $4 \cdot 1 \%$ & $1 \cdot 4 \%$ \\
\hline Pakistan & 67 & 56 & $56 \%$ & 48 & 28 & $1.1 \%$ & $3 \cdot 2 \%$ & $2 \cdot 1 \%$ \\
\hline Democratic Republic of the Congo & 37 & 56 & $60 \%$ & 161 & 69 & $-2 \cdot 4 \%$ & $5 \cdot 0 \%$ & $7 \cdot 4 \%$ \\
\hline Tanzania & 73 & 49 & $63 \%$ & 214 & 86 & $2 \cdot 3 \%$ & $5 \cdot 4 \%$ & $3.0 \%$ \\
\hline Mozambique & 44 & 48 & $66 \%$ & 246 & 163 & $-0.5 \%$ & $2 \cdot 4 \%$ & $2.9 \%$ \\
\hline Kenya & 51 & 43 & $69 \%$ & 163 & 86 & $1.0 \%$ & $3 \cdot 8 \%$ & $2 \cdot 8 \%$ \\
\hline China & 129 & 39 & $71 \%$ & 10 & $2 \cdot 7$ & $7.0 \%$ & $7 \cdot 7 \%$ & $0.7 \%$ \\
\hline Myanmar & 65 & 32 & $73 \%$ & 141 & 60 & $4 \cdot 2 \%$ & $5 \cdot 0 \%$ & $0.9 \%$ \\
\hline Ethiopia & 93 & 29 & $75 \%$ & 139 & 28 & $6 \cdot 9 \%$ & $9 \cdot 4 \%$ & $2 \cdot 6 \%$ \\
\hline Angola & 13 & 28 & $77 \%$ & 80 & 93 & $-4 \cdot 5 \%$ & $-0.9 \%$ & $3 \cdot 6 \%$ \\
\hline Philippines & 38 & 27 & $79 \%$ & 48 & 26 & $2.0 \%$ & $3.6 \%$ & $1.6 \%$ \\
\hline Uganda & 25 & 25 & $80 \%$ & 104 & 58 & $0.0 \%$ & $3 \cdot 4 \%$ & $3 \cdot 4 \%$ \\
\hline Zambia & 24 & 18 & $82 \%$ & 225 & 106 & $1.7 \%$ & $4 \cdot 4 \%$ & $2 \cdot 7 \%$ \\
\hline Ghana & 15 & 16 & $83 \%$ & 78 & 54 & $-0.4 \%$ & $2 \cdot 2 \%$ & $2 \cdot 5 \%$ \\
\hline North Korea & 56 & 16 & $84 \%$ & 120 & 63 & $7 \cdot 4 \%$ & $3 \cdot 8 \%$ & $-3 \cdot 6 \%$ \\
\hline Madagascar & 13 & 14 & $84 \%$ & 82 & 54 & $-0 \cdot 4 \%$ & $2 \cdot 5 \%$ & $2 \cdot 9 \%$ \\
\hline Cameroon & 24 & 13 & $85 \%$ & 157 & 55 & $3.6 \%$ & $6 \cdot 2 \%$ & $2.6 \%$ \\
\hline Thailand & 25 & 12 & $86 \%$ & 40 & 18 & $4 \cdot 3 \%$ & $4 \cdot 7 \%$ & $0.4 \%$ \\
\hline Russia & 32 & 12 & $87 \%$ & 22 & $8 \cdot 4$ & $5 \cdot 8 \%$ & $5 \cdot 7 \%$ & $-0 \cdot 1 \%$ \\
\hline Vietnam & 31 & 12 & $88 \%$ & 39 & 13 & $5 \cdot 6 \%$ & $6 \cdot 5 \%$ & $0.9 \%$ \\
\hline Somalia & 9 & 10 & $88 \%$ & 100 & 70 & $-0.6 \%$ & $2 \cdot 1 \%$ & $2 \cdot 7 \%$ \\
\hline Afghanistan & 14 & 10 & $89 \%$ & 67 & 29 & $2.0 \%$ & $4.9 \%$ & $3.0 \%$ \\
\hline Zimbabwe & 20 & $8 \cdot 3$ & $89 \%$ & 163 & 50 & $5 \cdot 2 \%$ & $7.0 \%$ & $1.8 \%$ \\
\hline Côte d'Ivoire & 25 & $8 \cdot 3$ & $90 \%$ & 153 & 34 & $6 \cdot 5 \%$ & $8.8 \%$ & $2 \cdot 4 \%$ \\
\hline Brazil & 10 & 7 & $90 \%$ & 5.9 & $3 \cdot 3$ & $2 \cdot 1 \%$ & $3 \cdot 4 \%$ & $1.3 \%$ \\
\hline Nepal & $4 \cdot 8$ & 6.9 & $91 \%$ & 20 & 24 & $-2 \cdot 1 \%$ & $-1.1 \%$ & $1.1 \%$ \\
\hline \multicolumn{9}{|c|}{$\begin{array}{l}\text { Countries are ranked from that with the highest number of deaths in } 2017 \text { (India) to that with the lowest number of deaths (Nepal). The following countries have achieved average rates of decline in death rates } \\
6 \% \text { or more annually from 2000-17: China. Ethiopia, Cameroon, Vietnam, Zimbabwe, and Côte d'Ilvoire. The following countries had an increase in the tuberculosis death rate from 2000-17: Angola and Nepal. } \\
\text { *Per updated classification in the WHO's global tuberculosis report," measures of mortality include tuberculosis deaths in individuals infected with HIV. †The death rate is expressed as tuberculosis deaths per } \\
100000 \text { people, per year. } \text { Average annual rate of decline from 2000-17 (\% per year); a negative rate of decline indicates an increase in death rate. \$Demographic headwinds is calculated as average annual rate } \\
\text { change in death rates minus average annual rate of change in deaths; it illustrates death rate changes even after accounting for population growth. }\end{array}$} \\
\hline
\end{tabular}

Ministry of Health and Family Welfare, New Delhi, India (R Rao MD); Office of Public Health Studies, University of Hawaii, Mānoa, HI, USA (V Fan); Department of Paediatrics, Center for International Child Health, University of Melbourne, Melbourne, VIC, Australia (S M Graham); Burnet Institute, Melbourne, VIC, Australia (S M Graham); National Tuberculosis, Leprosy and Lung Disease Program, Ministry of Health, Nairobi, Kenya (M Kamene MD, treatment, short course, or DOTS, as its solution to the problem. DOTS, which used direct observation to improve adherence to a rifampicin-based standardised treatment regimen of 6 to 9 months, also required diagnosing tuberculosis by sputum smear and reporting cases and treatment outcomes to public health authorities. The original DOTS framework focused on infectious, smear-positive cases. Although technical guidelines were subsequently published by WHO on all types of tuberculosis, DOTS did not specifically emphasise smear-negative tuberculosis, extrapulmonary tuberculosis, childhood tuberculosis, or drug-resistant tuberculosis; neither did it address latent tuberculosis infection. The DOTS approach, while perhaps fit to budget constraints, was therefore not comprehensive and proved insufficient to curtail ongoing tuberculosis transmission. The expanding HIV epidemic and the growth of drug-resistant tuberculosis further undermined the DOTS strategy, which was hampered by imprecise diagnostic tools and passive case detection.

Despite progress against the tuberculosis pandemic since the introduction of DOTS - and subsequently, an enhanced strategy by WHO to intensify tuberculosis control efforts ${ }^{2}$ - the potential to dramatically reduce tuberculosis incidence and mortality worldwide as first proposed in 1993 has not been realised. 


\begin{tabular}{|c|c|c|c|c|c|}
\hline & \multirow{2}{*}{$\begin{array}{l}\text { Total number of } \\
\text { deaths (thousands) }\end{array}$} & \multicolumn{3}{|c|}{ Deaths (\%) by age group in 2016} & \multirow{2}{*}{$\begin{array}{l}\text { Average annual rate of } \\
\text { decline (\% per year) } \\
\text { 2000-16 }\end{array}$} \\
\hline & & $0-4$ years & 5-69years & $\geq 70$ years & \\
\hline Tuberculosis* & 1290 & $1.9 \%$ & $70 \%$ & $28 \%$ & $1.4 \%$ \\
\hline HIV and AIDS* & 1010 & $6 \cdot 5 \%$ & $92 \%$ & $1 \cdot 3 \%$ & $1 \cdot 9 \%$ \\
\hline Diarrheal disease & 1380 & $35 \%$ & $36 \%$ & $15 \%$ & $2 \cdot 4 \%$ \\
\hline Vaccine-preventable disease & 274 & $41 \%$ & $48 \%$ & $11 \%$ & $4 \cdot 6 \%$ \\
\hline Meningitis and encephalitis & 383 & $26 \%$ & $58 \%$ & $16 \%$ & $2 \cdot 0 \%$ \\
\hline Malaria & 446 & $65 \%$ & $31 \%$ & $4.0 \%$ & $2 \cdot 6 \%$ \\
\hline Respiratory infections & 2970 & $30 \%$ & $24 \%$ & $46 \%$ & $0.7 \%$ \\
\hline \multicolumn{6}{|c|}{$\begin{array}{l}\text { *Per standard classification in the WHO's Global Health Estimate,' and in The Institute for Health Metrics and Evaluation Global Burden of Disease, tuberculosis deaths in } \\
\text { HIV-infected individuals are classified as AIDS deaths. }\end{array}$} \\
\hline
\end{tabular}

Dismayed by this lack of progress and after intensive collaboration with the global tuberculosis community, WHO proposed the End TB [tuberculosis] Strategy to the World Health Assembly that endorsed it in May, 2014. The new strategy was then incorporated into the UN Sustainable Development Goals (SDGs). By 2030, the strategy aims to reduce tuberculosis deaths to $90 \%$ of those in 2015 and tuberculosis incidence to $80 \%$ of that in 2015, and to ensure that families do not face catastrophic costs due to tuberculosis (appendix $\mathrm{p} 4$ )..$^{3-4}$ The global burden of tuberculosis in 2019 remains substantial and for reasons outlined below, those targets will not be attained without urgent corrective action.

\section{Tuberculosis-related mortality and the persistent burden of tuberculosis infection and disease}

Tuberculosis remains a global public health emergency, responsible for more deaths than any other infectious disease. Although globally the tuberculosis mortality rate has declined approximately $3 \%$ per year since 2000 , or $42 \%$ overall between 2000 and 2017, ${ }^{5}$ this decline reflects substantial progress in the number of patients diagnosed and treated. Moreover, it also occurred as poverty-related drivers of tuberculosis decreased and economies grew. For example, Côte d'Ivoire, Ethiopia, Vietnam, and Zimbabwe all achieved annual average rates of decline in tuberculosis mortality of more than $6 \%$ between 2000 and 2017 (table 1). This progress aside, however, tuberculosis deaths, especially among people with HIV and in children are still substantial. ${ }^{5,6}$ Furthermore, rates of tuberculosis mortality have declined much more slowly than for most other infectious diseases (table 2). In many parts of sub-Saharan Africa and southeast Asia, tuberculosis remains a leading cause of years-of-life lost. Moreover, tuberculosis ranks as the 13th leading cause of death and the 11th leading cause of years-of-life lost worldwide. ${ }^{8}$

An estimated 10 million people $(90 \%$ adults, $58 \%$ adult men) became ill with tuberculosis in 2017. Eight countries in southeast Asia and Africa
(India, China, Indonesia, Philippines, Pakistan, Nigeria, Bangladesh, and South Africa) accounted for two-thirds of all new cases worldwide. Overall, tuberculosis incidence has fallen approximately $1.4 \%$ per year since 2000 and $2 \%$ per year since 2015 . This is far less than the rate needed to achieve WHO End TB $\operatorname{targets}^{5}$ (an annual incidence rate decline of $4-5 \%$ by 2020 and $10 \%$ by 2025 to achieve the milestone case reductions) and less than declines in mortality. The overall slow decline in tuberculosis burden suggests that tuberculosis programmes, although reducing deaths, are insufficient to overcome poverty-related drivers that substantially affect the pandemic. ${ }^{9}$ Modelling studies suggest that, to avert transmission, individuals at risk must be identified and provided effective preventive therapy, and individuals with less infectious, early tuberculosis must be diagnosed and provided immediate treatment. ${ }^{10,11}$

Between 2000 and 2016, 32 national tuberculosis prevalence surveys were done in 26 countries. ${ }^{5}$ Many of these studies have found a higher prevalence of tuberculosis than previous estimates based on less precise information, such as case notifications. The upwardly revised incidence estimates highlighted large numbers of undiagnosed or unreported tuberculosis cases in many countries. Prevalence surveys also showed that people with tuberculosis often sought care for symptoms that health-care workers did not identify. Other individuals did not recognise the seriousness of their symptoms and had not sought care. All prevalence surveys in the past decade have found a higher burden of tuberculosis among men, with men/women ratios ranging from 1.2 (in Ethiopia) to 4.6 (in Vietnam). ${ }^{5}$ The higher global disease burden in men-estimated to be 1.8 times higher than in women ${ }^{5}$ - combined with larger detection and reporting gaps highlight gender differences in accessing care that might be related to both financial barriers and stigma. ${ }^{12}$ The differences also suggest that male-friendly strategies to improve access to and use of health services are required. ${ }^{13}$
EW Mailu MPH); WHO, Nairobi, Kenya (E O Masin MPH); Office of the Secretary-General's Special Envoy on Tuberculosis, United Nations, Geneva, Switzerland (L McHugh); International Institute of Social Studies, Erasmus University Rotterdam, The Hague, Netherland (E Mitchell PhD); Global Health Centre

The Graduate Institute Geneva, Geneva, Switzerland (S Moon); Linksbridge, Seattle, WA, USA (M Osberg MPP); Internationa Institute for Global Health, United Nations University, Kuala Lumpur, Malaysia (M Remme PhD); Seattle, WA, USA (G Stallworthy MHS); Department of Paediatrics and Child Health, Stellenbosch University, Stellenbosch, South Africa (J A Seddon); and National TB Program, WHO, Chisinau, Moldova (V Vilc MD)

Correspondence to: Dr Michael Reid, Division of Infectious Diseases, University of California San Francisco, San Francisco, CA 94143, USA michael.reid2@ucsf.edu See Online for appendix 


\section{Reasons for slow progress}

The slow progress against tuberculosis since 1993 has resulted from a mix of political, societal, scientific, and strategic shortcomings. These shortcomings include health system frailties; lack of investment in control efforts and in research towards developing new medical tools; reliance on simplified, one-size-fits-all approaches that do not meet the different needs of individual patients; biological factors, such as HIV coinfection and the spread of drug resistance; and the huge and persistent reservoir of latent tuberculosis infection. Moreover, tuberculosis disproportionately affects those communities with the least agency to effect change. The Lancet Commission on Investing in Health, ${ }^{14}$ in a report prepared for the 2018 Astana Conference on Health for All, assessed the progress against tuberculosis in the context of progress on other key outcomes, including child mortality, maternal mortality, and HIV. The Commission concluded that the rates of decline in child mortality and HIV mortality since 2000 were high, and still increasing. Progress against maternal mortality was slow and against tuberculosis slower still. In both cases, progress had slowed since 2010 . The challenge to the tuberculosis community stands clear.

\section{Insufficient investment and political will}

Deaths from tuberculosis decreased rapidly in western Europe and the USA as living standards improved. The combination of a decline in tuberculosis cases in highincome countries (HICs) and the absence of a powerful civil society voice in high-burden countries has undermined efforts to garner the same political support or domestic investment as for other diseases. Efforts have been hampered in low-income countries because of a failure to recognise the profound negative economic impact of the pandemic and to advocate for increased donor financing in high-burden. In many of the highest burden countries, chronic underfunding and absence of political will have profoundly disabled tuberculosis programmes, and also explain why, 40 years after the Alma Ata Declaration, ${ }^{15}$ half of the world's population still lacks access to comprehensive health-care services.

Funding for tuberculosis research and development has been stagnant for many years, despite tuberculosis remaining a major global health threat. ${ }^{3}$ A reflection of this underinvestment is the continued reliance upon tools such as smear microscopy and the BCG vaccine, which were developed nearly a century ago. ${ }^{16}$ Although global funding for tuberculosis research received more funding in 2018 than ever before (US\$772 million), the pace at which scientific discovery progresses has been greatly hindered by insufficient funding dedicated to research priorities that have been extensively defined. ${ }^{17-19}$

\section{Broken care cascades and poor quality of care}

Improvement of tuberculosis management requires early, accurate case detection together with the rapid initiation of and adherence to effective treatment that prevents
Mycobacterium tuberculosis ( $M$ tuberculosis) transmission, especially in high-burden countries. Therefore, national tuberculosis programmes in such settings must first invest to ensure that all patients with tuberculosis seeking care have access to diagnostics and treatments. Unfortunately, tuberculosis care is frequently delivered with little attention to patient needs and preferences, poorly coordinated with other services, and undermined by insufficient access to essential services. ${ }^{20} \mathrm{~A}$ recent assessment of patient pathways in 13 countries accounting for $92 \%$ of the world's missed tuberculosis cases showed that even among people who actively sought care, fewer than one-third sought care at a facility that had the capacity to diagnose or treat people with tuberculosis, or both..$^{20-23}$ Referral systems to access diagnostic technologies also were restricted. These findings confirm results from numerous other studies from various settings that show the many programmatic and financial barriers ${ }^{24,25}$ preventing people with tuberculosis from accessing health care. ${ }^{26}$ Furthermore, they highlight how it is crucial to align the availability of services to where people seek care.

Not only is access highly variable, so too is the quality of tuberculosis care in many high-burden countries. Although the DOTS strategy emphasised the importance of quality-assured drugs and diagnostics, it neglected to ensure the prioritisation of the quality of tuberculosis care. The Lancet Global Health Commission on highquality health systems, published in 2018, highlighted that half of all tuberculosis deaths result from poorquality care. ${ }^{27}$ As figure 1 shows, the quality of care is undermined by chronic underfunding, limited access to new tools, and the inadequate implementation of policies.

Numerous studies have highlighted substantial gaps in the tuberculosis care continuum for all forms of tuberculosis cases: active disease, drug-resistant tuberculosis, latent infection, and childhood tuberculosis. ${ }^{36-39,45}$ In an Indian analysis of patients with multidrug-resistant tuberculosis, only 14\% completed treatment and $11 \%$ remained disease-free at 1 year. ${ }^{37}$ One study in South Africa found that only $82 \%$ of the 532005 tuberculosis cases were diagnosed, and less than $54 \%$ of drugsusceptible tuberculosis cases completed treatment. ${ }^{38}$ Of those with rifampicin-resistant tuberculosis, only $22 \%$ completed treatment (appendix p 9). Standardised patient studies in three countries (China, India, and Kenya) show that most primary care providers are unable to diagnose tuberculosis. Moreover, referral links to the National Tuberculosis Programme are weak, with data from standardised patient studies in these three countries showing that only $28 \%$ to $45 \%$ of patients were correctly managed by primary care providers..$^{34,35,46}$

Simply put, the global capacity to diagnose, link to care, treat, and cure patients with tuberculosis is woefully inadequate for the massive burden of disease that exists. The public health implications, as well as the poor clinical and financial implications for patients, ${ }^{41}$ are 


\begin{tabular}{|c|c|c|c|c|}
\hline \multicolumn{5}{|c|}{ Quality of tuberculosis care: people-centred, equitable, resilient, and efficient } \\
\hline \multicolumn{2}{|c|}{ Process of care } & \multicolumn{3}{|c|}{ Quality impact } \\
\hline \multicolumn{2}{|c|}{$\begin{array}{l}\text { 2-month delay in diagnosis } \\
\text { Only } 1 \text { in } 2 \text { patients with drug-susceptible tuberculosis, } 1 \text { in } 5 \\
\text { patients with MDR tuberculosis, and } 1 \text { in } 5 \text { patients with latent } \\
\text { tuberculosis infection are adequately diagnosed and treated }\end{array}$} & \multicolumn{2}{|c|}{$\begin{array}{l}\quad \text { Delays in diagnosis results in } \\
\text { High costs to patients } \\
\text { (patients spend more than half of annual } \\
\text { income on care) } \\
\text { Increased waiting times for treatment }\end{array}$} & $\begin{array}{l}10 \text { million new cases, } \\
1.6 \text { million deaths (case } \\
\text { fatality } 16 \% \text { ) in } 2017 \\
558000 \text { new MDR or RR } \\
\text { tuberculosis cases, resulting } \\
\text { in } 230000 \text { MDR and RR } \\
\text { tuberculosis deaths }\end{array}$ \\
\hline $\begin{array}{l}\quad \text { Process of care } \\
50-60 \% \\
\text { patients begin seeking } \\
\text { care in informal (eg, } \\
\text { ayurvedic or homeopathic } \\
\text { doctors, and pharmacists) } \\
\text { and private sectors }\end{array}$ & $\begin{array}{l}\quad \text { Governance } \\
52 \% \text { HBCs recommend } \\
\text { Xpert MTB/RIF as initial test. } \\
47 \% \text { have implemented this } \\
\text { In } 8 \text { low-income HBCs, } \\
\text { domestic funding represents } \\
<7 \% \text { of NTP budget needs }\end{array}$ & \begin{tabular}{l}
\multicolumn{1}{c}{ Platforms } \\
1.1 microscopy labs \\
per 100000 population \\
$1 \cdot 3$ DST per 5 million \\
population \\
Limited accessibility to \\
tuberculosis services at \\
community level
\end{tabular} & $\begin{array}{l}\text { Workforce } \\
3 \text { health-care providers } \\
\text { are seen before diagnosis } \\
28 \%-45 \% \text { of providers } \\
\text { correctly manage } \\
\text { tuberculosis cases }\end{array}$ & $\begin{array}{l}\text { Tools } \\
10 \text { sputum smears for every } \\
\text { Xpert test in HBCs } \\
20 \% \text { of patients in need of } \\
\text { bedaquiline have received } \\
\text { it }\end{array}$ \\
\hline
\end{tabular}

Figure 1: Dimensions of tuberculosis care quality and barriers that undermine optimal service quality ${ }^{5,16,20,21,26-46}$

This figure, based on the framework used by Lancet Global Health Commission on High Quality Health Systems in the SDG Era, ${ }^{27}$ highlights how the quality of tuberculosis services is undermined when there is inadequate investment in foundational infrastructure, tools, and resources. DST=drug-susceptibility testing. $\mathrm{HBC}=$ high-burden countries. $\mathrm{MDR}=$ multidrug-resistant. $\mathrm{RR}=$ rifampicin-resistant.

self-evident. Substantially reducing tuberculosis mortality and incidence will require a great increase in both the coverage and the quality of tuberculosis services across the entire care continuum.

\section{Failures to optimise private sector engagement}

Of the 3.6 million unrecognised or missing patients with tuberculosis (ie, those patients that either do not present for diagnosis or who are diagnosed but whose disease is not notified to the tuberculosis programmes) in 2017, $56 \%$ of them were in seven countries where primary care is dominated by private providers and more than $75 \%$ of initial care-seeking is in the private sector (table 3). However, in these countries, private provider notifications are just $20 \%$ of total tuberculosis notifications and $12 \%$ of estimated tuberculosis incidence. Based on data from tuberculosis prevalence surveys and private sector drug sales, ${ }^{47}$ a considerable proportion of patients are treated in the private sector, with largely unknown levels of quality and patient outcomes. Given the dominance of private health care in countries with the largest share of missing patients with tuberculosis, private providers must be engaged to provide high-quality, person-centred care on a scale equal to their role in primary care to meet national and global goals.

Modelling studies also suggest that untreated or poorly treated patients in the private sector are a major source of $M$ tuberculosis transmission, ${ }^{48}$ which is due to delay in diagnosis and treatment initiation, as well as recurrent tuberculosis among patients who were inadequately treated in this sector. Therefore, improving the diagnosis and treatment of patients seeking care in private facilities is an opportunity to rapidly reduce tuberculosis transmission. Engaging private providers can also reduce unnecessary morbidity and mortality caused by inappropriate treatment, drug resistance caused by undetected multidrug-resistant tuberculosis and incomplete treatment, and catastrophic expenditures and impoverishment.

\section{Failure to target resources at hot spots and high-risk populations}

Global and regional data camouflage localities where the tuberculosis pandemic continues to grow unabated. Many different microepidemics exist, and the risk of both acquiring and dying of tuberculosis is unevenly distributed across society. Even adjacent neighbourhoods might have a markedly different prevalence, as recent analysis from Chennai, India, shows. ${ }^{49}$ Such regional variations reflect social and environmental determinants, which include living in densely populated areas $\mathrm{s}^{50-52}$ and working in occupations such as health-care or mining that increase the risk of tuberculosis. ${ }^{53-55}$ Accurate case detection together with rapid initiation of and adherence to effective treatment (both preventive and curative) that prevents transmission are required. Therefore, National Tuberculosis Programmes in high-burden regions must scale up active case-finding strategies for those people and populations at the highest risk, rather than relying on passive case finding alone. Unfortunately, active casefinding strategies, even in the highest risk populations, are not widely implemented because of cost concerns and lack of research consensus on what best practices should be included. ${ }^{56}$ 


\begin{tabular}{|c|c|c|c|c|c|c|c|c|c|}
\hline & \multirow{2}{*}{$\begin{array}{l}\text { Tuberculosis } \\
\text { incidence } \\
\text { (thousands } \\
\text { [rank]) in } 2017\end{array}$} & \multirow{2}{*}{$\begin{array}{l}\text { Missing cases } \\
\text { (thousands } \\
\text { [rank]) in } 2017^{*}\end{array}$} & \multirow{2}{*}{$\begin{array}{l}\text { Multidrug- } \\
\text { resistant } \\
\text { tuberculosis } \\
\text { cases } \\
\text { (thousands } \\
\text { [rank]) in } 2017\end{array}$} & \multirow{2}{*}{$\begin{array}{l}\text { Private share } \\
\text { of early } \\
\text { care-seeking }\end{array}$} & \multicolumn{3}{|c|}{$\begin{array}{l}\text { Tuberculosis notifications by } \\
\text { private for-profit providers, } 2017\end{array}$} & \multicolumn{2}{|c|}{$\begin{array}{l}\text { Private share of } \\
\text { tuberculosis treatment }\end{array}$} \\
\hline & & & & & $\mathrm{n}$ & $\begin{array}{l}\% \text { of total } \\
\text { notifications }\end{array}$ & $\begin{array}{l}\% \text { of } \\
\text { estimated } \\
\text { incidence }\end{array}$ & $\begin{array}{l}\text { Population } \\
\text { survey }\end{array}$ & Drug sales \\
\hline India & $2740(1)$ & $953(1)$ & $135(1)$ & $80 \%$ & 383784 & $20 \%$ & $14 \%$ & $46 \%$ & $54 \%$ \\
\hline Indonesia & $842(3)$ & $400(2)$ & $23(7)$ & $74 \%$ & 59549 & $13 \%$ & $7 \%$ & $46 \%$ & $51 \%$ \\
\hline Nigeria & $418(6)$ & $316(3)$ & $24(6)$ & $67 \%$ & 3975 & $5 \%$ & $1 \%$ & $22 \%$ & NA \\
\hline Philippines & $581(4)$ & $264(4)$ & $27(4)$ & $70 \%$ & 52375 & $16 \%$ & $9 \%$ & $21 \%$ & $43 \%$ \\
\hline Pakistan & $525(5)$ & $166(5)$ & $27(4)$ & $85 \%$ & 79332 & $22 \%$ & $15 \%$ & NA & $45 \%$ \\
\hline Bangladesh & $364(7)$ & $121(6)$ & $8(11)$ & $82 \%$ & 67332 & $28 \%$ & $18 \%$ & $30 \%$ & NA \\
\hline Myanmar & $191(10)$ & $61(13)$ & $14(8)$ & $78 \%$ & 18149 & $14 \%$ & $10 \%$ &.. &.. \\
\hline Total & 5661 & 2021 & 244 & $75 \%$ & 665489 & $20 \%$ & $12 \%$ &. &.. \\
\hline$\%$ of global total & $57 \%$ & $56 \%$ & $41 \%$ &.. & .. &.. &.. &.. & .. \\
\hline
\end{tabular}

\section{Neglect of tuberculosis control strategies}

Ending tuberculosis as a disease of public health significance must entail a comprehensive, cogent prevention agenda. Because the human reservoir of $M$ tuberculosis infection is substantial, ${ }^{57}$ predominantly asymptomatic, and long-lived, identifying individuals who are at highest risk of progression to disease, who would thus benefit the most from preventive therapy, is crucial. The benefits of preventive tuberculosis therapy have been known for more than 60 years. Pioneering studies in the 1950s and 1960s provided strong evidence of the efficacy of isoniazid in preventing active tuberculosis in children ${ }^{58}$ Alaskan Native populations, residents of congregate living facilities (such as psychiatric hospitals), and household contacts of patients with tuberculosis. ${ }^{59}$ Subsequent work has further documented the benefits of preventive therapy for individuals with evidence of recent infection, those with radiographic evidence of previous untreated tuberculosis, ${ }^{60}$ people with $\mathrm{HIV}^{61}$ recipients of immunosuppressive therapy, ${ }^{62}$ and other immunocompromised individuals. ${ }^{63}$

Large population-based studies of tuberculosis preventive therapy and mathematical models both suggest that preventive treatment of tuberculosis infection - as part of a comprehensive approach that includes active case-finding and prompt, effective treatment—can sufficiently reduce population-level transmission to interrupt the cycle of infection, illness, and death. ${ }^{6,65}$ Unfortunately, despite abundant evidence of its efficacy, the use of preventive therapy globally has been limited, ${ }^{66}$ because tuberculosis control programmes in low-income and middle-income countries (LMICs) have focused almost exclusively on detection and treatment of individuals with active tuberculosis disease.

\section{Drug-resistant tuberculosis}

Among the 558000 individuals estimated to develop rifampicin-resistant tuberculosis each year, most are thought to be infected with multidrug-resistant tuberculosis (resistance to both rifampicin and isoniazid). ${ }^{67}$ Despite this large burden, only one-quarter of the estimated number of individuals with multidrugresistant or rifampicin-resistant tuberculosis were diagnosed and notified in 2017.5 The remainder either form part of the so-called missing millions or were placed on largely ineffective first-line treatment in the absence of a drug-resistant tuberculosis diagnosis. Among those diagnosed, 87\% were reported to have been enrolled on treatment, with only $55 \%$ of these successfully treated. This simple cascade leaves only $12 \%$ of the global multidrug-resistant or rifampicin-resistant tuberculosis burden successfully treated. Although the variations in the prevalence of drug-resistant tuberculosis between countries are substantial, multidrug-resistant prevalence can vary by a factor of 10 at the subdistrict level and even more from one health centre to the next. ${ }^{68,69}$ The largest number of drug-resistant tuberculosis cases are in India (which along with other high-burden countries has witnessed the emergence of so-called totally drugresistant strains) ${ }^{70}$ and China (where one-quarter of all active tuberculosis disease cases are resistant to either isoniazid or rifampicin). ${ }^{71}$ Importantly, increasing evidence shows that the majority of drug-resistant tuberculosis cases reflect transmission rather than initial acquisition. ${ }^{2-74}$ Thus, a high priority for curbing drugresistant tuberculosis is to interrupt its transmission through early diagnosis and prompt initiation of effective treatment. ${ }^{75}$ In parallel, an urgent need exists to develop and trial preventive treatment strategies that are effective against drug-resistant forms of this disease. 


\section{Social determinants of the tuberculosis pandemic}

Fundamentally, tuberculosis is a disease of poverty. ${ }^{76-79}$ Most often it causes substantial losses in productivity for people already living in poverty (3-4 months of work) and their families ( $30 \%$ of yearly household earnings).$^{80}$ Social determinants that contribute to tuberculosis risk are linked both directly and indirectly to social and economic vulnerabilities." Surveys in seven countries show that patients who develop tuberculosis often face catastrophic costs ( $>20 \%$ of household income in LMICs) just to access care for diagnosis and treatment. ${ }^{24,25,81-84}$ In Vietnam, for example, $63 \%$ of tuberculosis-affected households had catastrophic costs, 38\% needed loans or sold assets (socalled dissavings), and $27 \%$ reported serious tuberculosisrelated financial burdens. ${ }^{75}$ Substantial social and economic burdens make patients with tuberculosis less likely to present for care, complete tuberculosis testing, and initiate and adhere to treatment, ${ }^{78,86}$ leading to increased $M$ tuberculosis transmission, morbidity, and mortality. ${ }^{87-93}$ The financial effects of tuberculosis are substantial and long lasting; as shown in panel 1 , individuals with this disease in rural India had severe financial hardship even 7 years after completing tuberculosis treatment.

As history shows, the global tuberculosis pandemic is not homogenous and characterised by a gradual decline in incidence. Rather it is a heterogeneous collection of microepidemics in which transmission in each setting is driven by different factors, ${ }^{102}$ from HIV-induced immune defects to inadequate diagnosis and treatment. ${ }^{103}$ In settings where increased attention and resources have been devoted to control tuberculosis (eg, New York [US], ${ }^{104}$ Alaska [US], ${ }^{105}$ and China), ${ }^{71}$ remarkable successes have been achieved. However, in regions where facilitators of transmission have been left unaddressed (eg, incarceration in eastern Europe), tuberculosis has resurged. To prevent resurgence, tuberculosis control programmes must anticipate and respond to dynamic demographic, environmental, and socioeconomic trends, mapping each microepidemic to clearly understand its drivers and how it is evolving. In addition, anticipating the threats of vulnerable aging populations, global proliferation of urban slums, and the increasing incidence of noncommunicable diseases, such as diabetes and chronic lung disease, is essential. In the SDG era, ending tuberculosis must be framed within a broader health and development agenda. ${ }^{106}$ This agenda includes understanding that reducing tuberculosis mortality and improving the health system are inextricably linked with ensuring gender equality (SDG 5), improving working conditions (SDG 8) and urban planning (SDG 11), and mitigating the effect of air pollution and food insecurity caused by climate change (SDG 13). Purely biomedical or public health solutions are not enough to end the tuberculosis pandemic; ${ }^{107}$ economic development and exigent investment in social policy strategies that can alleviate the drivers of this disease are also important.
Panel 1: Long-term economic impact of tuberculosis on households in India

Multiple studies document the often substantial financial outlays faced by patients with tuberculosis and their families as a result of catastrophic tuberculosis-related medical expenses. ${ }^{94-97}$ However, few studies document the long-term economic effects of tuberculosis on households or provide insights on how and how often tuberculosis causes impoverishment, and the potential for financial recovery. ${ }^{98,99}$

\section{What we did and found}

We analysed longitudinal data (26032 rural households) from the India Human Development Survey (IHDS) I (2004-05) and II (2011-12). We used multivariable regressions to characterise the relationship between tuberculosis, expenditures, and loan-taking in the short term and risks of impoverishment and debt in the long term (7 years later), adjusting for baseline household sociodemographics and health, as well as geographic and seasonal fixed effects. Moderately poor households ( $<\$ 3.10$ per day per individual) reporting a case of tuberculosis at baseline were more likely to be extremely poor $(<\$ 1.90$ per day per individual) 7 years later (36\% [95\% Cl 23-50]). Despite India's overall economic growth during this period, 7-year growth of real, non-medical expenditures was three times higher for otherwise similar households without tuberculosis at baseline compared with those with tuberculosis. High-interest loan-taking was an important impoverishment mechanism.

\section{What it means}

Households experiencing an active tuberculosis case at baseline are more likely years later to either remain poor or to become impoverished and indebted than comparable households without active tuberculosis. Tuberculosis adverse effects, which extend well beyond an individual patient's health, are often long-lasting. These findings underscore the importance of WHO's social protection goals, which highlight the potential economic benefits of expanding social protection and insurance ${ }^{96,100}$ and affordable credit to rural areas to prevent households affected by tuberculosis from remaining or becoming poor, thus interrupting the disease-poverty cycle (appendix p 61). ${ }^{101}$

\section{Global leaders have made a strong political commitment to ending the tuberculosis pandemic}

The UNHLM in September, 2018, endorsed an ambitious and powerful declaration to accelerate progress towards the goals outlined in the End TB strategy (panel 2). Together, programmatic innovations, new health technologies, sustained global economic growth, increasing commitment to attaining UHC, and mounting political momentum to definitively address tuberculosis can all contribute to achieving that goal. A long-term political pledge, however, requires a clearly defined endpoint and a roadmap for how to achieve it. For the purposes of this report, the Commission focused primarily on the goals outlined in the UNHLM declaration and the End TB strategy mortality target: a reduction by $90 \%$ from the worldwide mortality in 2015, which was about 24 tuberculosis deaths per 100000 population per year (including in people with HIV). We recognise that efforts to reduce tuberculosis mortality must occur concurrently with strategies that prevent ongoing transmission and lead to reductions in incidence. However, focusing on mortality rather than incidence is motivated by a desire to make the recommendations of the report relevant to a broad audience of policy makers and public health practitioners, for whom change in mortality is a more useful metric of progress than tuberculosis incidence. 
Panel 2: UN High Level Meeting on tuberculosis

On Sept 26, 2018, Heads of States and government representatives from all UN member states affirmed a political declaration to end the global tuberculosis pandemic, pledging to work together to accelerate national and global collective actions. Among the specific goals that Heads of States agreed to were:

- Commit to diagnose and treat 40 million people with tuberculosis by 2022, including 3.5 million children and 1.5 million people with drug-resistant tuberculosis

- Commit to prevent tuberculosis for those at most risk of developing the diseaseproviding tuberculosis preventive therapy to at least 30 million people by 2022, including 4 million children under age 5 years and 6 million people with HIV

- Commit to secure sustainable financing for tuberculosis research and development with the aim of increasing overall global investment to US $\$ 2$ billion annually, ensuring that all countries contribute appropriately to support quality research and development of new tools and the effective implementation of approved health technologies

- Commit to mobilise sufficient financing for universal access to quality tuberculosis prevention, diagnosis, treatment, and care, with the aim of increasing global investment for ending tuberculosis and reaching at least US $\$ 13$ billion per year by 2022

- Requesting WHO to develop a multisectoral accountability framework and ensure its timely implementation no later than 2019

The Commission concluded that achieving that goal within a generation and at a feasible cost is realistic in many settings, but it will require substantial investment in resources. Countries like Japan, ${ }^{108}$ China, ${ }^{109,10}$ and Peru ${ }^{111}$ have showed that rapid declines in tuberculosis mortality can occur with sufficient political will and financial investment, and when multisectoral steps to alleviate poverty occurred in tandem with efforts to reduce tuberculosis mortality. If other countries can replicate the trends in tuberculosis mortality decline achieved in these countries, then a $90 \%$ reduction in tuberculosis death rates within a generation (ie, by 2045) is possible in many settings (figure 2). For some high-burden countries, however, even sustained investment will be insufficient; transformative innovations in service delivery and increased investment in new tools is necessary to end the epidemic in these settings. Thus, our Commission set out to answer two questions as the foundation for creating a roadmap for countries to reduce tuberculosis mortality: how should tuberculosis high-burden countries and their development partners target their future investments to ensure that ending tuberculosis is achieved, and what policy priorities are necessary to ensure that the UNHLM political declaration leads to rapid and sustained progress towards ending the epidemic?

\section{Report roadmap}

Section 1 of this report highlights proven strategies to reduce tuberculosis mortality in high-burden countries. We focus first on high-priority strategies needed to close gaps in the care continuum, including person-centred approaches for diagnosis and treatment, active casefinding approaches to reach high-risk populations, and the urgent need to implement prevention interventions.
We emphasise the crucial need for new models of private sector engagement to deliver high-quality care and innovative ideas to optimise care for patients with drugresistant tuberculosis.

The challenge tuberculosis presents also has resulted from neglecting to identify tuberculosis research as an integral, crucial priority during the past 25 years. ${ }^{112}$ Although ending tuberculosis with existing tools is possible, new products are essential to reduce cost, simplify implementation, and accelerate progress. In section 2, we describe why available funding for tuberculosis research and development must increase to expedite transformative innovations in point-of-care diagnostics; safer, less toxic, and shorter treatment regimens than those currently available; chemoprevention; and a more effective tuberculosis vaccine. The economic rates of return on increased tuberculosis research and development investment are both substantial and invariably beneficial to poor and marginalised communities. ${ }^{113}$

Section 3 discusses how effective tuberculosis control represents one of the so-called best buys in global development, one that can produce considerable economic dividends for high-burden countries. We examine the potential to expand domestic tuberculosis financing through increased revenue generation and prioritising health care, as well as from more innovative sources, including loans, gains in efficiency, and complementary non-tuberculosis resources. Efforts to end tuberculosis within a generation need to differ dramatically from those in the past. Rather than relying on a global campaign funded and led by foreign donors and focused on specific interventions, increasingly tuberculosis control efforts will require domestic resources and full country ownership. ${ }^{114}$ We discuss how foreign donor support can still have a crucial role in transitioning countries to full country ownership by targeting resources to address drugresistant tuberculosis, investing in research and development, and strengthening strategies that ensure sustainable domestic funding for control efforts.

In section 4, we call for a new era of accountability and a reinvigorated cadre of political leaders committed to doing their part to accelerate efforts to end tuberculosis worldwide. Heads of states, national tuberculosis programmes, and even regional and site-level clinics must be held accountable for their performance in contributing to ending the epidemic. We advocate for an independent review mechanism to evaluate the performance of all major global stakeholders engaged in tuberculosis programming.

\section{Section 1: scaling up proven strategies}

Several high-performing countries have shown that substantive declines in tuberculosis mortality, although difficult to achieve, can be achieved by using existing tools to scale up evidence-based, best-practice interventions. To substantially reduce tuberculosis death rates, we must prioritise delivering person-centred and family-centred 


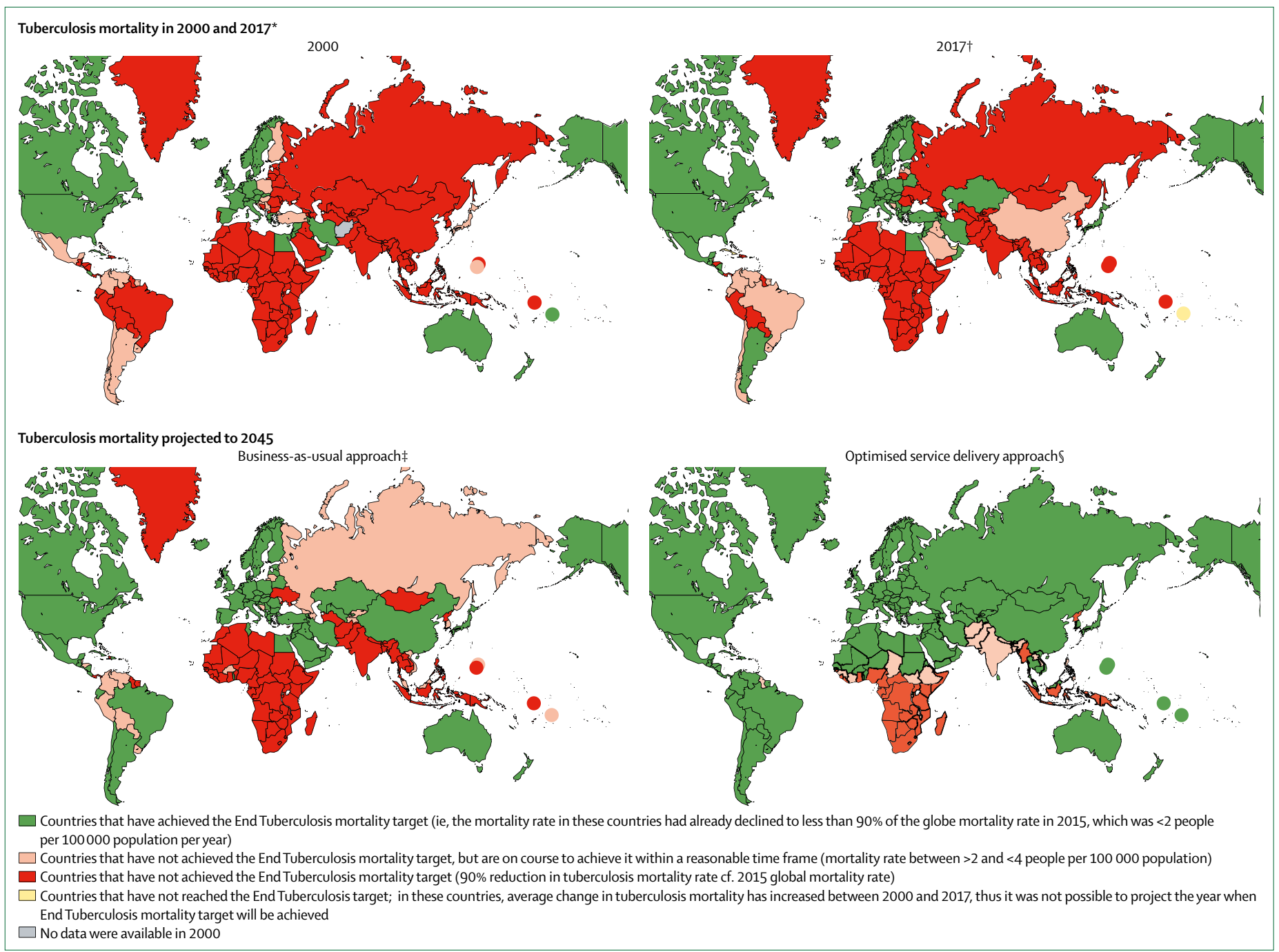

Figure 2: Progress toward End TB mortality target

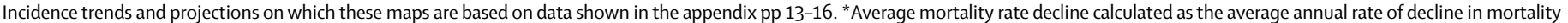

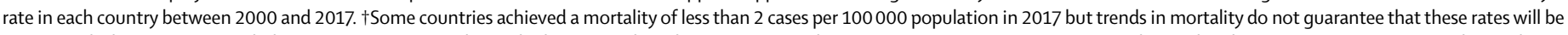

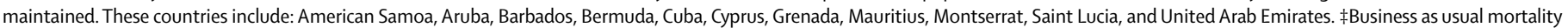

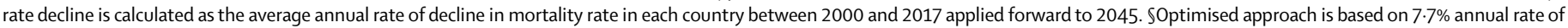
decline in mortality and is applied to all countries. This estimated decline in mortality has been achieved by highest performing countries from $2000-17$.

programmes to individuals with active disease, while also reaching high-risk populations with screening and preventive services. This comprehensive, integrated approach requires first focusing resources to ensure the availability of high-quality services to diagnose, treat, and prevent all forms of tuberculosis in both the public and private sectors. It then requires investing in strategies to find those with tuberculosis in high-risk communities and scaling up preventive interventions in these communities. Although no single approach is appropriate for all countries, we highlight policy priorities that can inform domestic budget allocations and donor investments in high-burden countries, and we also discuss the specific challenges faced by high-burden countries where private sector care is substantial. and where drug-resistant tuberculosis is prevalent or emerging. These recommendations are summarised in panel 3. To complement these recommendations, we present modelling analysis from three countries with different epidemiologic profiles, Kenya, India, and Moldova.

\section{Ensuring delivery of high-quality, person-centred services}

Defining person-centred care

To respond effectively to people with tuberculosis and to reduce delays in their diagnosis, treatment, and cure, tuberculosis services must be person-centred-that is, they must be holistic, individualised, empowering, and respectful, encouraging informed decision making and self-determination. ${ }^{115}$ Given that tuberculosis commonly 
Panel 3: Commission recommendations

In the wake of the UN High Level Meeting on tuberculosis on Sept 26, 2018, this Lancet Commission provides a roadmap for countries to follow as they tackle their individual tuberculosis epidemics. The roadmap outlines overarching policy goals and action steps that countries can take to reduce tuberculosis incidence and mortality.

\section{Scaling up proven strategies}

- Ensure person-centred and family-centred services are available to all who receive care for tuberculosis, guaranteeing access to high-quality diagnostics and treatment wherever they seek care

- Reach high-risk populations, beginning with those most easily identified through rapid screening, diagnosis, and robust treatment support; community engagement and adequate resources must be available to reach these populations

- Target certain high-risk populations and people for preventive therapy in tandem with active case-finding strategies; once high-risk populations are successfully reached, invest in identifying those with active tuberculosis in the general population, primarily by strengthening the capacity of health system delivery and moving toward Universal Health Coverage (UHC)

- Prioritise private provider engagement by building partnerships that improve quality of care and reporting of tuberculosis cases to encourage accountability, especially in high-burden countries that mostly provide care in the private sector

- Provide universal access to drug susceptibility testing (DST, as a minimum to rifampicin) at the time of diagnosis for all people with tuberculosis, and ensure access to second-line DST for all people with rifampicin-resistant tuberculosis

\section{Investing in tuberculosis research and development}

- Invest in and accelerate the pace of tuberculosis research, innovation, and development, including diagnostics, therapeutics, and chemopreventive strategies and vaccines, as well as population, policy, and implementation research; invest in research to overcome the challenge of tuberculosis and HIV co-infection because tuberculosis is the leading cause of death in people with HIV

- Invest in operational and programmatic research to rapidly translate research findings into tuberculosis control policies and programmes to address public health needs; these investments represent a global public good
- Implement and scale up the use of existing biomedical and prevention tools and strengthen the infrastructure and capacity to operationalise new research findings into tuberculosis control programmes

- Deliver strong advocacy to science ministries and research-oriented pharmaceutical companies, including ministries and companies in middle-income countries, to ensure global commitment to tuberculosis research and development; finance the early uptake of new products to provide important investment signals to product developers

Ensuring sustainable financing for tuberculosis

- Boost domestic resource mobilisation by increasing the distribution of public resources to health, pooling financing, and allocating tax revenues to health, especially in middle-income countries

- Reduce reliance on private finance of both private and public providers of tuberculosis services

- Increasingly focus donor financing for tuberculosis on investments in global public goods, including (but not limited to) market-shaping activities, support for tuberculosis advocacy and leadership, and research and development

- Continue donor financing for tuberculosis treatment and prevention as a priority in low-income countries, in addition to investing in reducing the spread, particularly the cross-border spread, of drug-resistant tuberculosis in all affected low-income and middle-income countries

- Develop new models of donor financing that catalyse domestic investment, encourage innovation, and strengthen accountability to citizens rather than donors

\section{Creating the enabling environment to End TB [tuberculosis]}

- Accelerate progress towards UHC; robust national tuberculosis programmes that can prioritise specific tuberculosis care, and prevention functions within a pathway to UHC are essential in high-burden countries

- Fortify the leadership and engagement of civil society in all aspects of tuberculosis programming by strengthening and increasing their decision making roles in policy, implementation, and accountability, and investing in their involvement as a global public good

- Establish independent, multisectoral accountability mechanisms, including the creation of report cards, to ensure that all parts, especially governments and their development partners, are accountable for progress towards ending the tuberculosis pandemic affects families, and young ( $<5$ years) and elderly ( $>70$ years) family members of people with tuberculosis are at high risk of developing tuberculosis disease, services must be family-centred ${ }^{116}$ in addition to person-centred. Thus, a thorough assessment of care-seeking behaviour, tuberculosis epidemiology, as well as local demographic and health system data, is necessary to determine where to prioritise resources and which delivery gaps ${ }^{117}$ to address first. In all contexts, the first priority must be ensuring universal access to high quality, person-centred tuberculosis care for individuals who are already in the health system.

Unfortunately, in many high-burden settings, health system frailties are inimical to delivery of person-centred tuberculosis services: individuals with tuberculosis often are neither identified nor appropriately evaluated in a timely manner; ${ }^{118,119}$ and once a diagnosis is established, 
they are not initiated on or supported to complete treatment that ensures a durable cure. Tuberculosis services must align with care-seeking behaviour to bring about person-centred care and prevention. Optimising alignment of services, both in national tuberculosis programmes and in the non-state sector (eg, private providers and nongovernmental organisations [NGOs]), can help ensure higher tuberculosis cure rates and improve the efficiency of care delivery to ensure greater equity and control costs. By redressing inequities in access, improving efficiencies in delivery, and protecting patients from physical and financial hardships, these interventions are also integral to robust health systems and to the broader the UN SDG agenda. ${ }^{120}$

\section{Rethinking tuberculosis service delivery}

As the UNHLM declaration showed, the political commitment to promote person-centred policies is strong. Solid ethical and moral rationales for adopting a people-centred approach to tuberculosis care also exist. Providing patients with choices about where they access care and giving them ownership over clinical decisions can have important beneficial clinical consequences, as efforts in Russia have shown. In one study, ${ }^{121}$ people who were lost to follow up in Tomsk, Russia, where alcohol abuse is a major comorbidity with multidrug-resistant tuberculosis, were offered alcohol reduction interventions along with nutritional support, transportation support, and a choice of where they would prefer to receive ongoing care (inpatient, day hospital, or at home). After the intervention, adherence improved from 52\% to $81 \%$ and a treatment success of $71 \%$ was achieved.

To be successful, person-centred tuberculosis care demands a radical rethinking of how treatment is delivered. Unfortunately, many national tuberculosis programmes have been slow to embrace new models of care, and have been constrained by limited technical capacity, scarce resources, and a myriad of competing priorities. This Commission emphasises that tuberculosis programmes need to learn to evolve continuously, responsive to changing demographics, patient preferences, and available data. Differentiated HIV service delivery has shown not only how service delivery innovations can improve efficiency and effectiveness, but also how communities can shape and inform systems. Similar strategies are necessary to transform tuberculosis service delivery. Marked disparities in particular demographic groups, such as the elderly (>70 years) and working-age men, highlight how the so-called one-size-fits-all strategy is untenable. The case for implementing responsive models of person-centred care that can reduce morbidity and end tuberculosis within a generation is clear. ${ }^{115}$

Aligning tuberculosis services with care-seeking patterns To realise the vision of sustainable health for all, we must ensure that health systems are fully resourced so all of those at risk of tuberculosis can access diagnostic, curative, and preventive services. Immediate and incremental steps are needed to strategically ensure that available resources are appropriately allocated, with a long-term goal of creating optimally integrated, person-centred health systems. To achieve these goals tuberculosis programmes must reallocate resources so that they align with how and where people with tuberculosis, and those at risk of developing the disease, seek care. Patient pathway analyses (PPAs) mapping the continuum of care for people with tuberculosis, using existing population-based surveys and routine programmatic data, can enable programmes to improve their understanding of how well patient careseeking and tuberculosis service availability align, highlighting system-level obstacles to patients accessing care. This step is essential to prioritise efforts and plan the placement of services to meet patient needs and preferences. This method is well characterised ${ }^{122}$ and, in 2017, results from five countries implementing PPAs and two countries implementing care cascades were published..$^{20}$ The analyses showed marked mismatches between diagnostic capability and tuberculosis careseeking behaviour, with less than $30 \%$ of facilities where patients initiate care able to do sputum smear microscopy and even fewer having the capacity to do an GeneXpert test or refer a sample for GeneXpert testing. ${ }^{21}$ These results also highlighted the need to prioritise deployment of rapid molecular tests in certain places and strengthen specimen referral mechanisms in others. In addition, PPAs have highlighted the importance of facility-level data to ensure efficient, targeted allocation of resources and to improve the primary health-care network to find the missing cases.

In 2016, WHO's Strategic and Technical Advisory Group for Tuberculosis recommended that all countries complete PPAs as part of their priority-setting and planning processes. ${ }^{123}$ Implementation guidelines have been published. However, fewer than ten countries have completed subnational PPAs or care cascades. ${ }^{122}$

Robust person-centred prioritisation and planning demands a change in how data is collated and translated. Myriad data collection requirements often leave national tuberculosis programmes with numerous data points that are disjointed, too many, and difficult to apply to decision making. Furthermore, in most settings, planning efforts have primarily used epidemiological data to inform resource allocation, rather than also considering how and where they should target resources to meet patient preferences. Several recent evaluations have enhanced our understanding of patient care seeking patterns and health system capacities. However, few of these data are being routinely incorporated into planning processes. Unfortunately, evidence generation has been heavily driven by top-down planning rather than by key programmatic questions from national tuberculosis programmes. In addition, donor requests for evidencebased plans are not harmonised or synchronised with country-level planning processes. Consequently, countries can be locked into perpetual planning cycles without time for implementation and learning, which 
makes a robust data consolidation process for each plan nearly impossible.

Designing person-centred programmes will require that data and evidence are consolidated so that gaps in the care continuum are identified. It also demands that tuberculosis survivors and their advocates have an integral role in how tuberculosis care programmes are designed, implemented, and evaluated. A systematic and uncompromisingly person-centred approach to the use of these data, as highlighted by the Kenya case study (appendix pp 20, 21) can enable national tuberculosis programmes to take the steps necessary to overcome the obstacles that prevent people with tuberculosis from reaching health services, not being diagnosed when they do reach a facility, or not being notified or completing treatment.

To support countries in moving toward person-centred planning, the global architecture of tuberculosis, including surveillance, technical assistance, and donor financing, will need to better align with this step-wise, person-centred approach. Global TB results frameworks do not monitor gaps closed along the patient pathway or specific health interventions optimised to the patient experience. To address this issue, PPAs need to be routinely deployed as key components of a package of evidence that informs priorities and donor assistance. Although it follows that realignment of resources with care-seeking behaviour should improve the efficiency of allocating national tuberculosis programme resources, further research is warranted to validate this assumption.

\section{Use network optimisation and big data analytics to ensure all patients have access to services}

Network optimisation is one strategy that can be used in high-burden countries to ensure that patients presenting with tuberculosis symptoms, many of whom drop out of the patient pathway during the diagnostic phase, ${ }^{40}$ have access to rapid and accurate diagnostic services. Borrowing analytic approaches from manufacturing industries, network optimisation involves selecting the best network configuration from available alternatives on the basis of selected criteria and subject to constraints. Applied to tuberculosis diagnostic services, it can help balance the need to increase access to diagnostic services for those most in need while ensuring cost efficiency and feasibility, informing instrument placement, sample transportation, referral mechanisms, staffing, and geographical prioritisation. Furthermore, by integrating data from other diagnostic tools (eg, chest radiography and HIV testing) and other disease programmes (eg, HIV care and treatment services), network optimisation can enable more precise resource allocation across health sectors and programmes.

One example of this approach comes from Lesotho, where diagnostic network mapping was used to analyse the national tuberculosis programme testing and care cascade, and inform procurement decisions. Despite a high unmet need, less than half of GeneXpert testing capacity was being used in 19 of 25 sites where it was available. Initially the national tuberculosis programme planned to procure and deploy additional instruments within the network. However, an analysis found that network capacity could be better optimised by improving referral flows and adjusting the placement of existing instruments: relocating 13 existing instruments would have the equivalent effect as the planned procurement of seven new instruments. ${ }^{124}$

In the near future, big data aggregated from routine Ministry of Health reports, donor-agency operating plans, private health systems, and social media, as well as other sectors of government, will help transform the efficiency of tuberculosis programmes, enabling targeted scale up of services and providing unprecedented situational awareness and analytic capability to Ministers of Health and national tuberculosis programmes managers. At present, examples of aggregated data being used to enhance the delivery of person-centred programmes are scarce in resource-limited settings. However, integrated data platforms, in combination with simulation technology, could enable national tuberculosis programmes to create detailed real-time models of the tuberculosis case continuum, incorporating variability in patient careseeking behaviours, diagnostic capacities, gaps in linkages, and health-care costs. In the future, such data systems could provide user-friendly dashboards at each level of the health system, with a single interface for both static and real-time analysis of complex systems, enabling national tuberculosis programmes to predict changes in patient-demand, anticipate stock-outs, determine use of diagnostic and treatment assets and, ultimately, improve patient care. The use of aggregated, big data sources will demand specialised equipment, interoperability standards, coherent data collection, and analysis systems, as well as regulatory oversight. ${ }^{125}$ However, these approaches are being successfully applied to address other complex health system problems in the USA ${ }^{126,127}$ and elsewhere. ${ }^{128}$ Certainly such innovations could successfully help close delivery gaps for tuberculosis programmes, especially if used in tandem with technologies that empower patients. The disruptive opportunity of smartphones, for example, to increase service demand generation, enhance provider accountability, ${ }^{129}$ and optimise adherence, ${ }^{130,131}$ are substantial. ${ }^{132}$ The effect of these digital solutions is likely to be incremental and heterogeneous, exaggerated in the short term but underappreciated in the long run. ${ }^{125}$

\section{Improving quality management to ensure high-quality service delivery}

In addition to PPA and network design analyses to ensure access to services for all patients presenting with tuberculosis, we must improve the quality of care that patients receive. Unfortunately, cascade of care analyses shows large gaps in the quality of care for both adults 
and children, and for both drug-susceptible and drug-resistant tuberculosis in many high-burden countries. ${ }^{36-38,112,133}$ Standardised patient studies in India, Kenya, South Africa, and China ${ }^{33-35,46}$ have shown that the quality of care for tuberculosis is poor. In a study in China, for example, health-care providers did not correctly manage patients presenting with archetypal symptoms or results suggesting active tuberculosis $59 \%$ of the time..$^{35}$ In an Indian study, only one-third of private practitioners correctly managed tuberculosis when presented with a text-book standardised patient with this disease. ${ }^{33,46}$

Traditionally, programmatic effects and outcomes have been defined primarily by epidemiological measures. Such a focus, however, overlooks that outcomes tied to improving care quality by closing gaps along the care cascade are more relevant operationally and can accelerate progress. Quality management tools can help frontline providers and national tuberculosis programme managers address those gaps to improve care quality, as well as address the drivers of ongoing transmission..$^{134}$

Quality management programmes must become part of national tuberculosis programmes and ideally integrated into existing national quality management programmes (appendix p 26). Ensuring that national tuberculosis programmes managers and their teams have access to this expertise will facilitate the development of ways to measure and improve quality. Nonetheless, a culture change in how tuberculosis data are used to improve care must occur at every level of the health system, including greater accountability of local tuberculosis clinics to patients they serve. Globally, a quality management programme that embraces improvement methods can be a powerful lever to improve donor-recipient accountability and enhance donor efficiency. WHO has a crucial role in supporting a quality management agenda and creating a global culture that supports quality improvement and accelerates dissemination of learning through peer exchange. Linking donor support to quality indicators could also improve efficiencies in donor financing and enhance transparency.

\section{Implementing quality improvement: lessons learned from tackling HIV}

Over the past few decades, HIV programmes in subSaharan Africa, the Caribbean, and Asia have implemented quality management programmes to optimise the use of limited resources available from governments and donor agencies. ${ }^{135}$ The basic elements of quality management include a formal quality management plan, a technical working group or committee, a set of performance measures, expectations for implementing quality improvement activities, staff capacity building, and patient or community involvement. These elements are necessary to achieve sustainability in the face of expected staff turnover and environmental changes that affect the stability of health-care organisations and the workforce. By leveraging a four-step continuous cycle of improvement (plan-do-check-act), these programmes have driven substantive change by developing local solutions to improve the quality of HIV care. Improvements have been shown across different facets of care, including treatment adherence, ${ }^{136}$ reducing mother-to-child transmission of $\mathrm{HIV},{ }^{137}$ paediatric services, ${ }^{138}$ enhancing adherence to treatment guidelines, ${ }^{139}$ and strengthening the clinical capacity of front-line providers. ${ }^{140}$

Similar approaches can be used to improve the quality of care for patients with tuberculosis, while also enabling increased accountability at all levels of national tuberculosis programmes (case studies in the appendix pp 22-24 provide examples from the public and private sector, at facility and regional level, of how quality improvement approaches have been deployed to improve tuberculosis outcomes).

Using the cascade of care as an organising framework, ${ }^{44}$ national tuberculosis programmes can measure quality at a facility-level with a set of indicators that represent key steps in the care cascade or that reflect the international standards of tuberculosis care (appendix p 25). ${ }^{141}$ National reporting of these quality indicators can help national tuberculosis programmes identify low-performing facilities that might require more support or resources. Furthermore, health facilities can use the tools of root cause analysis to identify specific barriers and generate ideas to address them.

However, as pointed out by the Lancet Global Health Commission on high-quality health systems ${ }^{27}$ improving quality will require system-wide action that goes beyond facility-based quality improvement efforts. These actions include better governance for quality; adopting competency-based clinical education and training in ethics and respectful care; and creating demand for quality in the population to empower people so they can hold systems accountable and actively seek high-quality care.

\section{Assessing the effect of strategies to deliver high-quality person-centred services}

Together, the strategies described in this section share the common objective of accurately diagnosing tuberculosis as early as possible: they reflect ways of realising the maximum potential effect of a system of tuberculosis services that is contingent on cases presenting for care. Modelling analysis, commissioned for this report, provides some insight on the potential value of these and other measures in three different country settings, each with distinct challenges in tuberculosis control: India (with a large private sector), Kenya (with HIV coinfection), and Moldova (with a high burden of multidrug-resistant tuberculosis). The full analysis is provided by Vesga and colleagues ${ }^{142}$ in a modelling study done in collaboration with this Commission. The example of Kenya is shown in figure 3: in this setting, patient pathway analysis has already identified the scarcity of diagnostic facilities as a key challenge. ${ }^{22}$ The figure shows the potential effect of measures that could increase the probability of diagnosis per provider visit to $90 \%$ : the effect is to reduce cumulative 

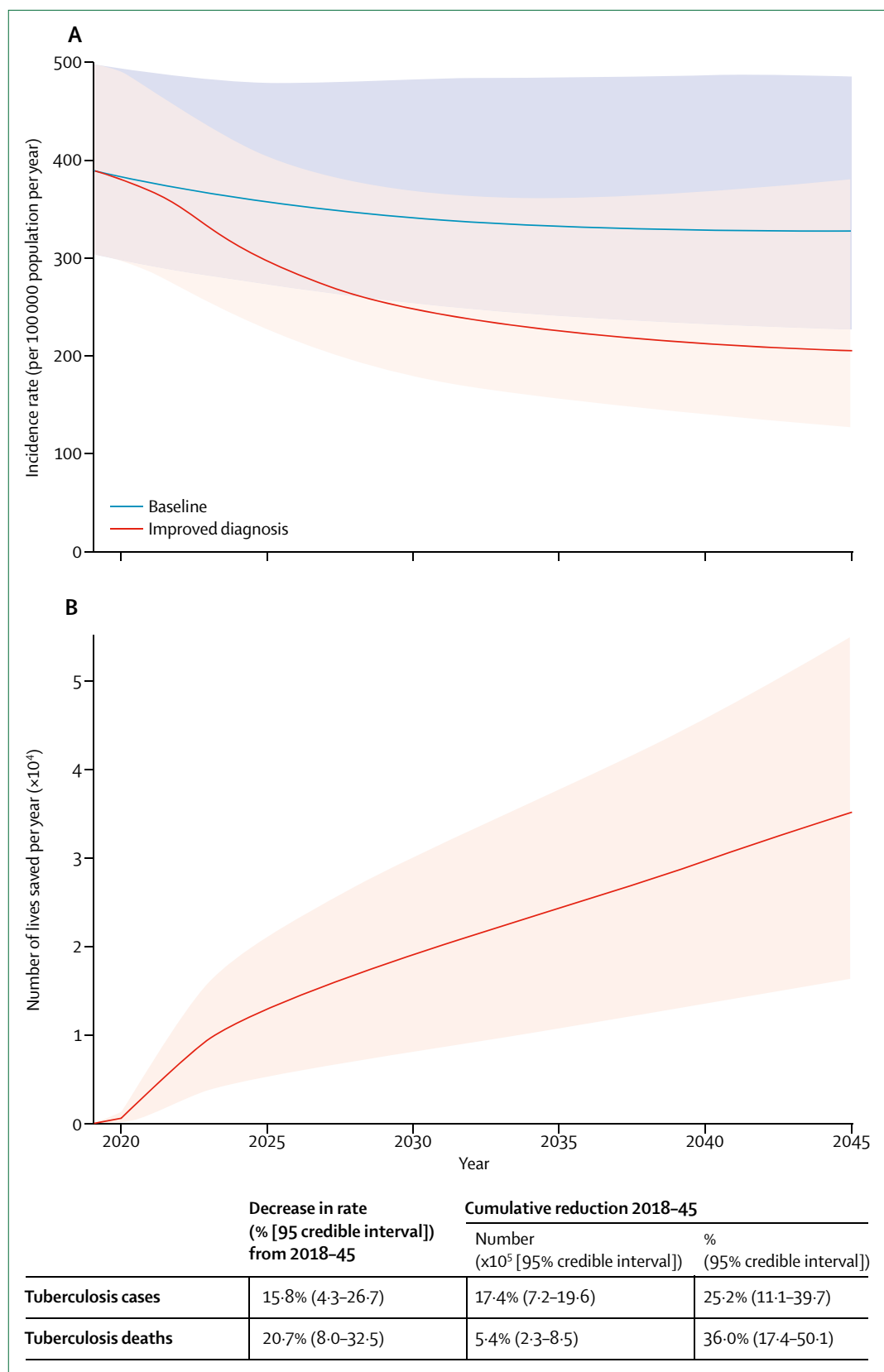

Figure 3: Incidence rates and lives saved in Kenya under an intervention of improved tuberculosis diagnosis (A) Tuberculosis incidence rate (per 100000 population per year) and (B) lives saved rate (per 100000 population per year). Blue line corresponds to a baseline scenario where all conditions remain unchanged, whereas the red line shows the results of an intervention scenario, with the probability of accurate diagnosis per patient-provider visit increased to $95 \%$. Detailed methods used to model these scenarios are available in Vesga and colleagues. ${ }^{142}$

tuberculosis cases from $2018-45$ by $25 \%$ (95\% credible intervals 11-39), and cumulative mortality over this time period by $36 \%$ (17-50). As described in this section, such measures are not limited to diagnostic tools: they also involve network optimisation, correcting misalignments of tuberculosis services, and other measures to maximise the effective uptake of rapid, accurate diagnostics. As the modelling shows, these measures are necessary but insufficient to end tuberculosis. However, in concert with the other strategies outlined in section 1, they can enable countries to make substantial progress towards ending the epidemic.

\section{Prioritised active case finding}

Besides targeting resources and analyses to ensure highquality, person-centred care for those individuals with tuberculosis, another high priority is finding people with tuberculosis, especially among high-risk populations, who have not yet presented for care. Strategies to find these missing patients must occur together with scaling-up access to preventive interventions. These two strategiesactive case-finding and prevention-must be programmatically inseparable and not separated by budget allocation decisions. Although active case finding mainly seeks early detection of and prompt treatment for people with active tuberculosis, thereby reducing mortality, morbidity, patient costs, and ongoing transmission, it also aims to identify people eligible for treatment of latent tuberculosis infection. ${ }^{143}$

\section{Active case-finding: closing the know-do gap}

Prevalence surveys in high-burden countries ${ }^{144-146}$ provide abundant evidence that despite scaling up and decentralising tuberculosis diagnosis and treatment services, undetected cases remain an important problem, especially for high-risk groups. ${ }^{147-150}$ Unfortunately, most high-burden countries have not widely implemented strategies to find these individuals because of insufficient funding, political will, and scientific consensus. ${ }^{151-153}$ As a result, the impact of active case-finding strategies on tuberculosis epidemiology in high-burden settings is limited; only a few studies have been published, with mixed results. ${ }^{152-155}$ Nonetheless, available clinical research, ${ }^{156}$ mathematical modelling, ${ }^{157,158}$ and considerable programmatic experience ${ }^{158,159}$ suggest that these strategies can be taken to scale. In Russia, in 2015, almost one half of the tuberculosis burden was detected by actively screening $68 \%$ of the prison population. In Brazil, tuberculosis screening of the prison population yielded 6021 new cases, $8 \%$ of the total national burden in $2015 \cdot{ }^{160}$

Although implementing active case-finding requires a systematic approach, ministries of health and their partners also need to consider how to scale up targeted active case-finding interventions. Important considerations include setting clear goals and objectives on the basis of a thorough assessment of the situation; identifying and prioritising risk groups; and choosing simple algorithms and accurate, effective technologies. ${ }^{159,161}$ In addition, consideration should be given to use best practices to disseminate innovations; ${ }^{117,162}$ establish and use networks for change; actively engage the community; and ensure strong leadership and governance to guarantee the success of active case-finding activities. Linking these strategies to accountability frameworks and funding predicated on meeting case-finding targets might also have a role. 


\section{Prioritising high risk groups}

Several groups with diseases or exposures that put them at high risk for tuberculosis should always be systematically screened (appendix p 27). Among them, household contacts must always be a priority for screening programmes, given the strength of evidence showing the effect of strategies targeted to them. ${ }^{163}$ The importance of a family-centred approach-and recognition that tuberculosis is a disease that affects families, as much as it affects individuals - has important implications for active case-finding, so national tuberculosis programmes need to recognise the family, not the individual, as the unit of intervention.

Other risk groups might warrant targeted screening programmes based on epidemiology, health system capacity, availability of resources, and feasibility. Given higher incidence of tuberculosis in men compared with women in almost all high-risk groups, ${ }^{13}$ men-friendly strategies, such as workplace interventions, should be employed where feasible. In preparing active case-finding scale-up strategies, the risk of discrimination and stigmatisation should be carefully addressed. In addition, the legal status of migrants, with regard to both access to health services and risk of expatriation in case of a tuberculosis diagnosis, needs to be considered. ${ }^{164}$ Engaging with civil society groups to improve the understanding of the expectations and concerns of high-risk groups when planning and implementing tuberculosis screening activities is crucial to their success.

Opportunities for integrating active case finding with other essential services for these populations should be exploited when possible, especially when high-risk groups are already served by vertical, facility-based programmes ${ }^{165}$ or private providers ${ }^{166}$ and when active case-finding activities can be aligned with other health promotion activities. ${ }^{167}$ For some high-risk populations - such as people living in slums and the homeless -innovative, multipronged casefinding strategies, leveraging mhealth technologies, and incorporating social protection strategies might be necessary to maximise yield and rationalise costs ${ }^{166,168}$

Active case finding alone will be insufficient to eliminate tuberculosis in high-risk populations. Even if more individuals with tuberculosis are identified in at-risk populations, those patients will return to their high-risk pools where the prevalence of tuberculosis risk factors is high. A multisectoral approach is essential to ensure that drivers of tuberculosis risk, such as malnutrition and air pollution, are addressed. It is also essential that active case-finding interventions are programmatically inseparable from interventions targeted at preventing tuberculosis disease in those latently infected and at greatest risk of developing active disease.

\section{Anticipating costs and using planning tools}

Scaling up active case-finding strategies will require substantial additional resources. The cost of screening can be high for each case identified, ${ }^{169-171}$ especially when compared with other health promotion interventions. ${ }^{172}$ Nonetheless, evidence on the cost-effectiveness and benefits of expanded financing for active case finding suggests that such investments will yield a high return. Modelling done as part of the South African government's investment case for tuberculosis (figure 4) show that the decline in tuberculosis transmission resulting from high case detection and optimal treatment will be highly costeffective if major and durable reductions in tuberculosis incidence and prevalence are achieved. Other modelling studies that include the benefits from reduced transmission also confirm that even where active screening costs are high, active case-finding strategies still can be highly cost-effective. ${ }^{157,171}$

Planning tools, such as the WHO's online ScreenTB tool, ${ }^{174}$ can help national tuberculosis programmes plan their case-finding activities and prioritise risk groups for screening by modelling the potential case yields and costs of different screening approaches. The ScreenTB tool allows the user to select risk groups of interest and compare estimates of the yield of screening (including true-positive and false-positive cases found), the total costs, and the cost per case detected across the selected risk groups and across different screening algorithms.

\section{Leveraging technology to improve the efficiency of case-finding strategies}

The tools used to screen for and diagnose tuberculosis are crucial in determining the efficacy of systematic screening. A rapid triage test that would enable active screening in the community would be a more efficient, person-centred approach to case-finding than available approaches and warrants substantial investment (appendix p 28). Mobile, automated, digital chest radiography units to detect lung lesions in people who are relatively asymptomatic ${ }^{175,176}$ might also help detect many more patients with tuberculosis than is possible through passive case-finding or self-reporting. Although data are sparse,${ }^{177}$ computeraided detection tools, used in concert with digital radiography, could substantially increase diagnostic sensitivity while also saving money. Clearly, this technology will also enhance sensitivity for detecting other pathologies, in addition to pulmonary tuberculosis, underscoring the importance of incorporating active case-finding in the setting of comprehensive primary care services.

In addition to new diagnostic technologies, improved use of available data-aggregated and anonymised, and collected from a variety of sources, including social media, pharmacies, ${ }^{47}$ and the private sector-has the potential to enhance both the precision and efficiency of active case-finding interventions, especially strategies that extend from high-risk populations and into low-risk communities. Already, social network data, mobile phone records, and spatial data have been combined to improve HIV testing proportions in $\mathrm{Uganda}^{178}$ and to show that imported malaria contributes substantially to disease burden in urban centres in Kenya. ${ }^{179}$ The effect of 


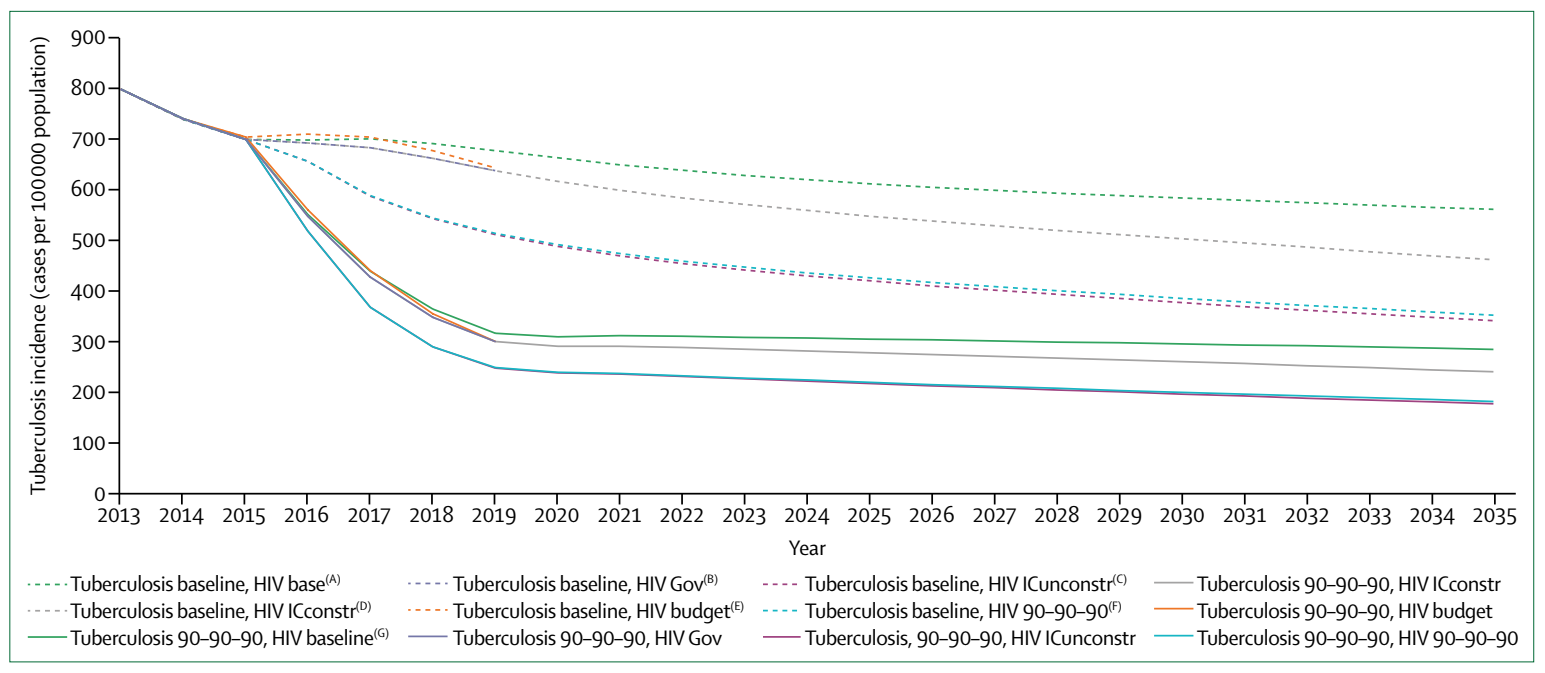

Figure 4: South African investment case for tuberculosis

(A) Baseline scenario (ie, business-as-usual). (B) Government (Gov) targets: current mix of interventions against HIV and tuberculosis over the next 5 years, at current coverage targets endorsed by the government. (C) Optimisation scenario without constraints (ICunconstr; ie, the most efficient mix of interventions against HIV and tuberculosis and without budgetary limitations). (D) Optimisation scenario with budget constraints (ICconstr; ie, outcomes are assessed with the current budget envelope). (E) 90-90-90 budget scenario, which was adapted for a more budget-relevant scenario. (F) Optimisation towards $90-90-90$ targets. The most cost-effective package of interventions to achieve the 90-90-90 targets. (G) 90-90-90 scenario that aims to detect and treat $90 \%$ prevalent tuberculosis cases by 2020 , with $90 \%$ of those on treatment achieving treatment success. Reproduced by permission of Minister of Health from South Africa. ${ }^{173}$

these additional data to address tuberculosis active casefinding efforts will be small unless they can be captured and integrated into existing data systems.

\section{Finding cases in low-risk populations}

Reaching the general population through active casefinding should remain a low priority until high-risk populations are successfully covered. Nonetheless, recognising that active case-finding is a high-value intervention, both epidemiologically and economically, low-risk populations in high-burden countries should not be ignored. The identification of the most effective mix of interventions and strategies that national tuberculosis programmes can use to detect patients in both highrisk and low-risk populations, and the empowerment of national tuberculosis programme managers to select the most appropriate combination of approaches in their unique settings, are key for success. Within a country, different provinces or districts might use various methods, depending on population sociodemographic, civil society engagement, and health system assets. Selecting appropriate interventions and strategies hinges on a rigorous, ongoing process of scientific research, knowledge sharing, and monitoring and evaluation.

\section{Prioritising tuberculosis prevention}

Tuberculosis prevention is a crucial but neglected component of global control of the disease pandemic. For the past 50 years, strategies for controlling tuberculosis in high-burden countries have focused on passive case detection and treatment of active disease, despite numerous policy recommendations advocating for increased attention on case detection interventions. ${ }^{158,159,180,181}$
Mathematical modelling shows focusing on passive strategies alone, while averting deaths and decreasing morbidity, will not end tuberculosis. Rather, ending tuberculosis will require multiple different preventive interventions to interrupt transmission, treat latent infections, immunise close contacts, and treat or prevent comorbidities, such as HIV, that increase susceptibility to develop active disease. Table 4 shows some populations that might benefit from prevention interventions. ${ }^{185}$ Tuberculosis infection control in health-care facilities and congregate settings (eg, prisons) is also especially important to tuberculosis prevention efforts: health-care centres and hospitals are often hotspots of tuberculosis transmission, and instituting environmental control measures and rigorous administrative and personal protective strategies is likely to reduce the transmission risk substantially. ${ }^{186}$

\section{Targeting preventive therapy}

Tuberculosis preventive therapy probably offers one of the most effective interventions to reduce tuberculosis incidence globally. In addition, by preventing tuberculosis and reducing mortality through the treatment of those with latent infection who are greatest risk of developing active disease, tuberculosis preventive therapy is a necessary component of a comprehensive strategy to end the pandemic. Even improved strategies for diagnosis and treatment will not address the large reservoir of latently infected people (estimated to be approximately 2 billion globally) who might develop tuberculosis at any point in their lifetimes. ${ }^{57}$ Clearly targeted tuberculosis preventive therapy could substantially reduce incidence of tuberculosis disease in the highest-risk groups. These groups 


\begin{tabular}{|c|c|c|c|c|}
\hline & Risk for progression & Global numbers & Responsibility for delivery & Reference \\
\hline $\begin{array}{l}\text { Patients with HIV } \\
\text { infection }\end{array}$ & 2-10\% per year & 30 million people & National AIDS programmes & UNAIDS ${ }^{182}$ \\
\hline$<5$ household contacts & $5-40 \%$ over 2 years & $\begin{array}{l}\sim 5.5 \text { million people } \\
\text { peryear }\end{array}$ & $\begin{array}{l}\text { Primary health care, tuberculosis programmes, } \\
\text { maternal and child health programmes }\end{array}$ & Dodd and colleagues ${ }^{183}$ \\
\hline$>5$ household contacts & Up to $10 \%$ over 2 years & $\begin{array}{l}\sim 20 \text { million people } \\
\text { per year }\end{array}$ & $\begin{array}{l}\text { Primary Health Care, national tuberculosis } \\
\text { programmes }\end{array}$ & $\begin{array}{l}\text { Fox and colleagues, }{ }^{152} \text { Cavalcante } \\
\text { and colleagues;, }{ }^{153} \text { Morrison and } \\
\text { colleagues }^{184}\end{array}$ \\
\hline $\begin{array}{l}\text { Health-care workers in } \\
\text { high-burden settings }\end{array}$ & $1-3 \%$ per year, variable & >10 million people & National and local health systems & .. \\
\hline $\begin{array}{l}\text { Prisoners in high-burden } \\
\text { settings }\end{array}$ & $1-10 \%$ per year & >10 million people & Correctional authorities &.. \\
\hline
\end{tabular}

include people with HIV; household and other close contacts of people with infectious tuberculosis; and people working or living in settings that foster the transmission of $M$ tuberculosis, such as congregate living settings, prisons, health-care facilities, ${ }^{187,188}$ and underground mines, especially those with silica exposure, which in itself greatly increases risk. ${ }^{149,189}$ Moreover, the process of providing tuberculosis preventive therapy will uncover active cases, as candidates for preventive therapy undergo screening to rule out disease before beginning treatment, which identifies previously undetected cases of tuberculosis disease.

Although the effectiveness of preventive therapy in preventing active tuberculosis is well established, ${ }^{60}$ public health programmes have prioritised tuberculosis casefinding and treatment rather than implementing this inexpensive and highly effective intervention. HIV programmes have focused primarily on rolling out lifesaving antiretroviral therapy, not least because of compelling evidence of its efficacy as tuberculosis prevention intervention. ${ }^{190,191}$ Studies have shown that tuberculosis preventive therapy using isoniazid substantially reduces mortality in people with both early and advanced HIV infection. ${ }^{192-194}$ Globally, results from modelling studies show that wide uptake of tuberculosis preventive therapy, coupled with improved case-finding and treatment, is more important than an effective vaccine for reaching tuberculosis elimination by $2050,{ }^{194}$ and that household contact evaluations and use of this therapy would avert 99000-117000 deaths per year in children younger than 15 years. ${ }^{195}$ These data underscore the importance of a family-centred approach to tuberculosis care to ensure that these contacts are routinely screened as part of the routine management of all people diagnosed with tuberculosis.

Numerous obstacles have hindered the scale-up of preventive therapy, and innovative approaches must be taken to overcome these barriers. ${ }^{185}$ Improved diagnostic tests to document tuberculosis infection, including point-of-care tests, would facilitate treatment of infection in people with an increased risk of developing tuberculosis, such as household contacts, although tuberculosis contacts that are children ( $<5$ years) and all people living with HIV in high-burden areas could potentially be treated without testing. Prognostic biomarkers that identify people with latent infections who are most likely to progress to active disease would allow more targeted use in high-risk populations and broader use of preventive therapy in low-risk populations. Global supplies of essential drugs, such as isoniazid, and newer drugs, such as rifapentine, are unreliable, and stock-outs are frequent; therefore, improving the supply chain of inexpensive and qualityassured drugs is crucial. The duration of preventive therapy using isoniazid, 6-9 months, often results in non-adherence and is leading to widespread concerns, largely unfounded, ${ }^{196}$ about preventive therapy causing drug resistance. Novel short-course regimens, such as 12 weeks of weekly rifapentine and isoniazid, or a 4-week regimen of daily rifapentine and isoniazid, could transform prevention efforts ${ }^{197-199}$ and reduce the risk of resistance emergence, while also saving money and lives. ${ }^{197,200,201}$ Nonetheless, rather than waiting for new diagnostics and shorter courses, this Commission asserts that national tuberculosis programmes should increase access to preventive therapy now. While scarce, there are examples of how national tuberculosis programmes and their partners have successfully implemented tuberculosis preventive therapy at scale (appendix pp 30-32).

To realise the full effect of preventive therapy, national tuberculosis programmes must secure resources to ensure that active case finding and preventive therapy are integrated into existing programmes for specific high-risk populations. Integrating tuberculosis screening and preventive services into care for people with HIV is particularly important, especially given extensive, highquality research showing the life-saving benefits of this strategy. ${ }^{192,202}$ Global efforts to provide antiretroviral therapy have now reached 20 million individuals with HIV, but another 17-19 million remain untreated. Fewer than 4 million people with HIV have ever received tuberculosis preventive therapy, highlighting the opportunity to substantially scale-up this intervention. Not scaling up this 


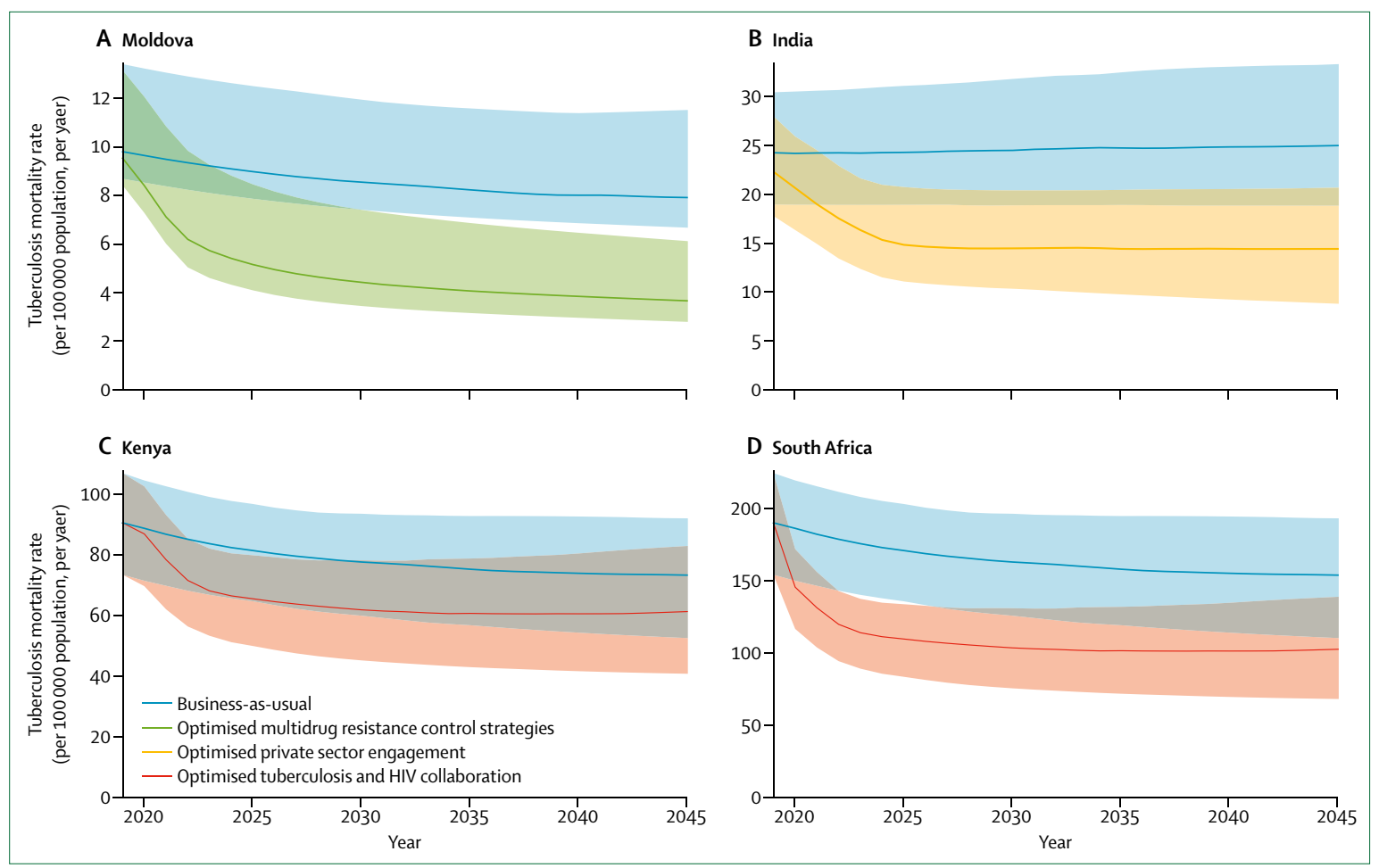

Figure 5: Potential lives saved from tuberculosis in different country settings

Mortality projections under intervention scenarios specific to each of the four high-burden countries shown. (A) Potential effect of improved multidrug-resistance control in Moldova, which has a high burden of multidrug-resistant tuberculosis. Increasing early drug susceptibility testing to $90 \%$ of all diagnosed tuberculosis cases and increasing second-line treatment success to $85 \%$ with new regimens would reduce multidrug-resistance incidence by $43 \%$ (95\% credible intervals $34-51$ ) in 2045 compared with 2015, and would avert 73\% (66-80) of tuberculosis deaths. (B) Potential effect of engaging the private sector in India, if tuberculosis care is optimised among $90 \%$ of private health-care providers. Through a combination of subsidised tuberculosis diagnostics and adherence support mechanisms, tuberculosis care in the private sector in India is assumed to be improved to the same standard as in the public sector. These measures will avert $28 \%$ of tuberculosis deaths ( $95 \%$ credible intervals 17-36) between 2018 and 2045; they will involve a mean incremental cost of US\$ 290 million per year (85-645) between 2018 and 2045, excluding the costs of managing drug-resistant tuberculosis. (C) Potential effect of improved collaboration between HIV and tuberculosis programmes in Kenya and (D) in South Africa. Mortality projections in these two high HIV burden scenarios are shown, with settings consistent with Kenya and South Africa, where the proportion of people coinfected with tuberculosis and HIV are $16 \%$ and $60 \%$, respectively. In both settings, increasing antiretroviral therapy coverage from current levels to $90 \%$ and increasing isoniazid preventive therapy amongst those in HIV care from current levels to $90 \%$ would reduce mortality by $33 \%$ (95\% credible intervals $18-49$ ) in Kenya, and by $46 \%$ (30-62) in South Africa. In the example of Kenya, these measures will involve a mean incremental cost of US\$66 million (95\% credible intervals $20-113$ ) per annum between 2018 and 2045. Cost estimates exclude the costs of managing drug-resistant tuberculosis. Inclusion of these costs is likely to lower the incremental cost, owing to the reduced burden of multidrug-resistant tuberculosis. Detailed methods used to model these scenarios are available in Vesga and colleagues. ${ }^{142}$

therapy for people living with HIV has probably caused several million deaths over the past decade. ${ }^{202}$

The analysis by Vesga and colleagues aimed to determine the effect of tuberculosis preventive therapy using isoniazid as recommended in countries with high rates of tuberculosis and HIV coinfection. By increasing tuberculosis preventive therapy among people living with HIV in Kenya to $90 \%$ (figure 5), tuberculosis mortality could be reduced by $17 \%$ between 2018 and 2045. In South Africa, a similar increase in preventive therapy coverage would lead to an even greater reduction in mortality over the same time frame. To achieve this effect, as well as to extend preventive therapy to other eligible groups recommended by $\mathrm{WHO},{ }^{203}$ will require additional investment. The incremental cost to the tuberculosis programme of increasing preventive therapy in Kenya and South Africa would be relatively modest (in Kenya, it is estimated that US\$66 million per yearbetween 2018 and 2045 would be necessary to achieve the results highlighted in figure 5), especially when compared with the economic costs of avoidable deaths resulting from not implementing this strategy. The efficiency of that investment can be enhanced by optimal use of health systems data to enable national tuberculosis programmes and their partners to plan interventions and monitor the effect of prevention strategies. ${ }^{204,205}$ Tuberculosis report cards tracking progress on these data at regional and local levels might also help accelerate tuberculosis preventive therapy scale-up efforts and ensure that national tuberculosis programmes and their partners are more accountable to civil society organisations and funders. The success of scale-up tuberculosis preventive therapy efforts will be contingent on recognition of the importance of shared responsibility from across health programmes and community stakeholders. 


\section{Importance of private provider engagement: from acknowledgment to prioritisation}

In most LMICs, private providers are an important source of health care for people of all socioeconomic groups, often offering accessibility and convenience not provided in the public system. Strictly speaking, private is synonymous with non-state and includes the for-profit as well as the non-profit sectors (ie, NGOs and faith-based organisations [FBOs]). Although most countries could improve their engagement of public and NGO and FBO providers, engaging for-profit private providers, which is even more important for tuberculosis control, has been much more difficult. In this section, we discuss some reasons for the failure to engage private providers, recent progress in how they can be engaged on a large scale for tuberculosis care, and the crucial actions countries must take to prioritise private provider engagement as part of their tuberculosis programmes. We highlight strategies to enable high-quality tuberculosis care in the private sector, opportunities for greater synergy between national tuberculosis programmes and private providers, and how the extended capability that the private sector provides can be leveraged to find those people with tuberculosis disease that are being missed by these programmes surveillance efforts.

\section{Making engagement of private providers a priority}

The need to engage private providers for tuberculosis control has been acknowledged in various global strategies since the early 1990s. ${ }^{206}$ Unfortunately, national tuberculosis programmes and their development partners have not focused sufficiently on engaging private providers in tuberculosis, and resources have not been adequate to meaningfully tackle this issue. The Global Fund to Fight AIDS, Tuberculosis, and Malaria (GFATM), which provides $56 \%$ of international development assistance for tuberculosis, had allocated less than $5 \%$ of grant budgets to engage a range of non-national tuberculosis programme providers defined as part of the public-private sector's collaboration. ${ }^{207}$ Because the GFATM responds to country requests for how its grant funds will be used, ultimately this small percentage reflects the low priority that countries place on the engagement with private providers. Although data on how much national programmes spend to engage private providers are scarce, an example from India is illustrative: before 2009 , only $1.5 \%$ of the state tuberculosis expenditure was allocated to engage NGOs and private providers. ${ }^{208}$

Poor engagement with private providers is often attributed to national tuberculosis programme staff shortages, but clearly the constraints are multifactorial. ${ }^{209}$ Most health systems in LMICs are weak in areas essential for effective private provider engagement, such as regulatory enforcement, strategic purchasing, and health information systems. National programmes often have insufficient basic information on the number of private providers, their role in tuberculosis patient care-seeking, and the drivers of patient and provider behaviours. Therefore, the national programmes managers find it difficult to engage hundreds and thousands of independent private providers with widely varying capabilities. For their part, private providers are often wary of engaging with government programmes and, given competitive market dynamics and financial imperatives, unwilling to adhere to guidelines promoted by national programmes.

Failure to meaningfully engage private providers reflects a strong preference for the public sector among those who manage tuberculosis programmes, those who fund them, and those offering technical support. The tuberculosis community has successfully embraced many innovations, including new diagnostics, treatment tools, and approaches to address tuberculosis and HIV coinfection and multidrug-resistant tuberculosis. These innovations, however, could be adopted in the public sector without challenging the basic public-sector business models. In contrast, private provider engagement can succeed at scale only when national tuberculosis programmes acknowledge that they cannot continue with the available business-as-usual model (table 5). Nonetheless, such engagement must occur simultaneously with strategies that protect patients and their families from severe financial losses that can arise from accessing care in the private sector. ${ }^{210,211}$ In working towards ending the tuberculosis epidemic in countries with a large private sector, it will be essential to protect the interests of people living in poverty by ensuring that public resources are applied to reduce user fees, while leveraging the private sector to expand diagnostic and treatment coverage.

\section{Catalysing progress and new opportunities to engage private providers}

Although private provider engagement in tuberculosis is far from adequate, considerable experience has accrued regarding how to successfully engage private providers of care. ${ }^{212}$ Many small, externally supported pilot projects to engage private providers have been implemented over the years. A study in 2006 reviewed data from 15 projects in eight countries, ${ }^{213}$ a systematic review in 2011 considered 45 studies from 22 projects in 12 countries, ${ }^{214}$ and another study in 2016 found 78 studies reporting on 48 programmes in 16 countries. ${ }^{215}$ Most projects did not reach substantial scale or could not be sustained over long periods. Nevertheless, these projects have generated abundant evidence that engaging private providers can substantially increase tuberculosis case detection and achieve treatment success rates that are at least as good as those in the public sector. Data on cost-effectiveness, financial protections, delays to treatment, and reaching the poor is less robust but also available. ${ }^{216}$ New research continues to add to our understanding of the functioning of private health-care markets regarding tuberculosis..$^{34,217,218}$ 


\begin{tabular}{|c|c|c|}
\hline & Available operating model & Disruptive operating model \\
\hline Implicit mission and role & $\begin{array}{l}\text { Care for patients with tuberculosis who seek care at public facilities; } \\
\text { act as service provider }\end{array}$ & $\begin{array}{l}\text { Care for all patients with tuberculosis regardless of where they seek care; act as } \\
\text { service enabler }\end{array}$ \\
\hline Service delivery alignment & $\begin{array}{l}\text { Public sector providers and facilities responsive to national } \\
\text { tuberculosis programmes direction }\end{array}$ & All providers and facilities, prioritised according to patient preferences \\
\hline $\begin{array}{l}\text { Approach to private providers and } \\
\text { markets }\end{array}$ & $\begin{array}{l}\text { Compete with and mistrust of private providers; deny or combat } \\
\text { market forces }\end{array}$ & $\begin{array}{l}\text { Collaborate and partner with private providers as indicated by their role in } \\
\text { health market system; acknowledge and harness market forces }\end{array}$ \\
\hline Mental map for engagement & $\begin{array}{l}\text { Preference for standardisation; top-down, policy-driven innovation; } \\
\text { linear systems }\end{array}$ & $\begin{array}{l}\text { Comfort with ambiguity and adaptation; innovation as emergent evolutionary } \\
\text { process; complex adaptive systems }\end{array}$ \\
\hline Tools for engagement & Mainly internal: forms, standardised procedures, and instructions & Mainly external: incentives, enablers, and contracts \\
\hline Core skills and competencies & Line management, supervision, and logistics & Negotiation, influence, and purchasing \\
\hline Core skills and competencies & Staff and leadership medical & $\begin{array}{l}\text { Staff and leadership with a balanced expertise including social science, health } \\
\text { systems, and health finance }\end{array}$ \\
\hline Data for decision making & $\begin{array}{l}\text { Overwhelmingly medical data: service statistics on case-notification } \\
\text { and treatment outcomes; weak or inconsistent data on source of } \\
\text { notification and referral; no data on resource allocation by type of } \\
\text { provider }\end{array}$ & $\begin{array}{l}\text { Balance of medical and socioeconomic data, including: numbers, characteristics, } \\
\text { location, and tuberculosis services of all kinds of providers; patient care-seeking } \\
\text { pathways and preferences; provider behaviours and motivations; private drug } \\
\text { sales volumes and trends; resource allocation by type of provider }\end{array}$ \\
\hline Funding for private provider engagement & $\begin{array}{l}\text { Ad hoc, short-term, small-scale, and external; optional extra, first to } \\
\text { be cut }\end{array}$ & Integrated into core budgets; long-term; large-scale; domestic and external \\
\hline Financing modalities & Input-based, supply-side, historical & Performance-based, often demand-side \\
\hline
\end{tabular}

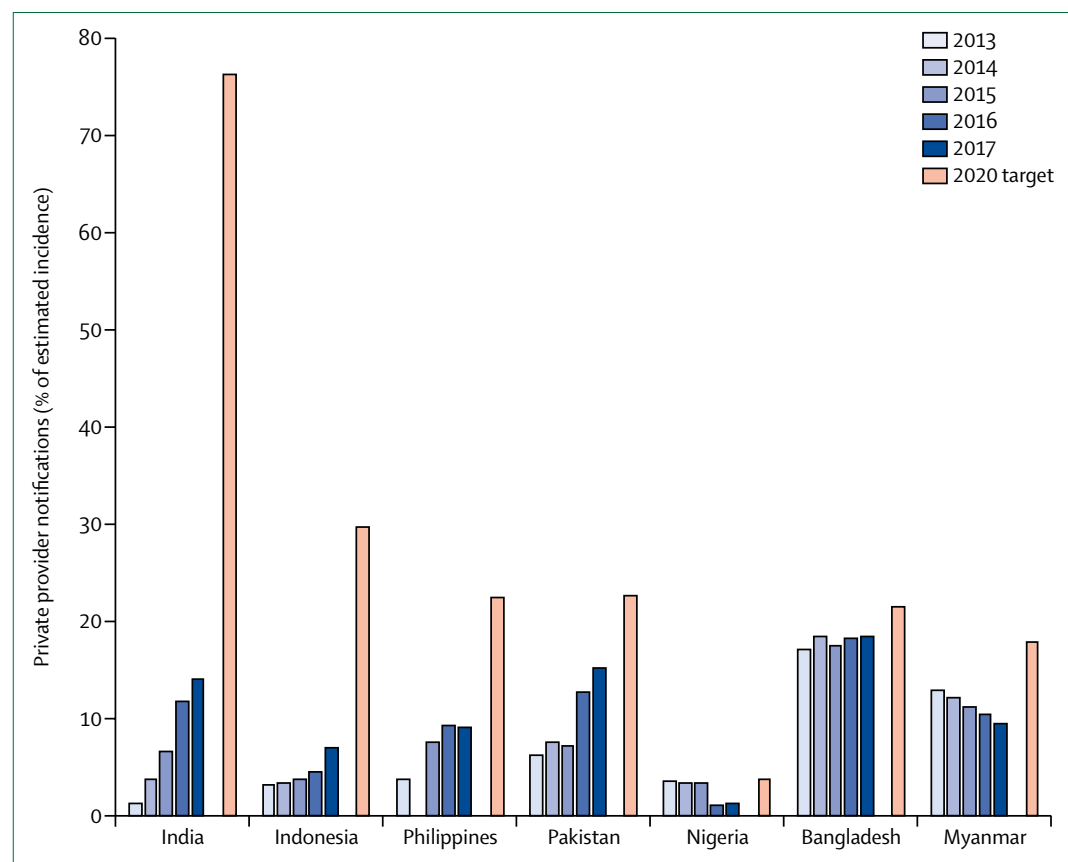

Figure 6: Percentage of privately notified patients with tuberculosis among the total estimated number of incident cases of tuberculosis, 2013-17 and target for 2020, in seven high-burden countries with dominant private health-care systems
Pakistan, and the Philippines increased their engagement of private providers, with private notifications increasing to $149 \%-153 \%$ of incident cases in 2017 , whereas private notifications in Indonesia increased to $7 \%$ of estimated incidence in 2017. Nigeria made no progress, with private notifications remaining in the range of $1 \%-3 \%$ of estimated incidence during this period.

In Bangladesh, Myanmar, and Pakistan, engagement of large numbers of private primary care providers has been led by strong NGOs acting as intermediaries between providers and the national tuberculosis programmes. These mission-driven NGOs have identified enhancing private provider engagement for tuberculosis as part of their long-term role and have succeeded in attracting resources from multiple donors to sustain their work. Some organisations are generalist NGOs, such as Bangladesh Rural Advancement Committee and Mercy Corps in Pakistan; others are more focused on tuberculosis, such as Damien Foundation in Bangladesh and Interactive Research and Development in Pakistan; Greenstar in Pakistan and Population Services International in Myanmar are social marketing organisations that have long engaged private markets for family planning and other health issues. All these organisations have in common an understanding of private providers, the ability to operate at scale, strong management systems (for human resources, information, and logistics), dynamic leadership, an aptitude for adaptation and innovation, and success in fundraising.

Efforts in Indonesia and the Philippines have focused on private specialists and hospitals rather than primary care providers. The national tuberculosis programmes have partnered with specialist-led associations (eg, the Indonesia Pulmonologist Society and the Philippines Tuberculosis Society). However, much of the initial 
care-seeking and tuberculosis treatment in these countries are among private primary care providers and, therefore, more effort to engage these providers will be needed. Social health insurance schemes, approaching full population coverage in both countries, are contracting with an increasing number of private providers for primary care services. Yet collaboration between the national programmes and social health insurance remains quite restricted. ${ }^{17}$

One of the most exciting developments is the political commitment in 2017 in India to scale-up private provider engagement nationwide, building on the success of several large demonstration projects (appendix p 33). ${ }^{18}$ India's National Strategic Plan for Tuberculosis Elimination (2017-25) commits to a large expansion of private provider engagement and calls for a six-time increase in private notifications to 2 million patients per year by 2020 , which would represent $75 \%$ of the estimated tuberculosis incidence. If India's plan succeeds, it will be the first major high-burden country with a dominant private health-care sector to align its tuberculosis programme with the care-seeking patterns of its population. Private notification targets for Bangladesh, Pakistan, and the Philippines are much more modest: $18-24 \%$ of estimated tuberculosis incidence by 2020 (figure 6). Overall, at least ten countries have recently prepared product portfolio management action plans, ${ }^{19}$ and the latest round of GFATM funding (2018-20) includes substantial components for private provider engagement in several countries.

As successful evidence on private provider engagement is reported, defined packages of interventions could be disseminated as templates that could be adapted for rapid scale up..$^{20}$ The core interventions in such templates include defined activities to engage private providers (eg, stakeholder consultation, provider mapping and prioritisation, relationship management, facilitating reporting of tuberculosis cases and data, and patient support for adherence); addressing financial and nonfinancial incentives for private providers; and ensuring private patients have access to high quality drugs and diagnostics according to national protocols. Although intervention packages can and have been summarised in general terms, continued innovation and adaptation should be encouraged.

In addition, legal and regulatory frameworks should be in place to ensure tuberculosis notification and quality services by private providers. Several countries have reissued laws and regulations requiring providers to report cases, sometimes conditioning relicensing and accreditation to tuberculosis notification. ${ }^{21}$ Although regulatory penalties might have a role to play, countries most successful in engaging private providers have invested more in enablers (such as call centres to facilitate notification) and incentives (such as easy access to drugs and diagnostics) while respecting private providers' interests. Professional societies can be and have been successfully engaged to help define best practices for tuberculosis among private providers.

Looking ahead, new opportunities and developments could enhance private provider engagement for tuberculosis in the coming years. First, success in a country like India could set an example that inspires other countries. Second, the digital revolution is finally reaching tuberculosis. The use of information and communication technology (ICT) systems, coupled with call centres, can facilitate the engagement of private providers and provide digital, case-based information on patients with tuberculosis treated in the private sector to national tuberculosis programmes and experts in tuberculosis. Third, such information and communication technology systems can enable additional innovations that further facilitate private provider engagement at scale, such as digital vouchers for drugs and diagnostics, adherence monitoring technologies, and digital payment of incentives and enablers to both patients and providers. Fourth, access to new and improved diagnostic and treatment tools, such as digital chest X-rays and GeneXpert MTB/RIF, increased the value to private providers of engaging with the public sector. Finally, the emergence of social health insurance schemes for UHC offers an unprecedented platform to engage private providers at scale across all health conditions and provides an opportunity to improve quality and access of both curative and preventive tuberculosis services in the private primary sector in countries like Indonesia and Philippines. ${ }^{219}$

The challenges of optimising private sector to deliver high tuberculosis quality care, while protecting patients from excessive out-of-pocket expenditure, are considerable. To be successful these models must minimise fee-forservice payments that reward quantity over quality and do not promote high value, low cost interventions, such as tuberculosis preventive therapy. Nonetheless, as part of a broader UHC agenda, leveraging private sector services to provide public-financed services might enable extended capability while also accommodating the preferences of those most at risk for, or with, tuberculosis. ${ }^{220}$

\section{Modelling the effect of optimal private sector engagement}

Because of the large burden of tuberculosis that is managed in the private sector globally, it is essential to assess the effect of improving private sector engagement. Modelling commissioned for this report assessed how greater private sector engagement in a high-burden country like India, where private providers offer extended capability, could affect tuberculosis incidence and mortality. In such a setting, strategies to improve quality of private sector care, through a combination of subsidised tuberculosis diagnostics and adherence support mechanisms, tuberculosis care in the private sector would avert $28 \%$ of tuberculosis deaths over the next 30 years, saving an additional 8 million lives, beyond those lives saved by full implementation of other 
evidence-based interventions (figure 5). The additional cost of optimised private sector engagement would involve an annual increase of US\$290 million in national tuberculosis programme costs. Although this strategy alone would not be enough to end the epidemic in India, it has the potential to substantially reduce the public health threat posed by tuberculosis. Further, enhanced private sector engagement in concert with other strategies to close gaps in the care cascade, such as targeted active case-finding interventions, optimisation of diagnostic networks, and improved adherence support strategies, could lead to substantial reductions in tuberculosis mortality over the next 30 years..$^{142,221}$

\section{Tackling drug resistance}

Projections show that, over the next decade, at least 6 million people will develop drug-resistant tuberculosis. Without improvements in treatment provision and success for drug-resistant tuberculosis, many of these people will die from tuberculosis, with many transmitting their drug-resistant infections to others. ${ }^{5,222}$ By 2050, onefourth of the predicted 10 million annual deaths attributable to antimicrobial resistance globally are expected to be due to drug-resistant tuberculosis, which will make it the leading cause of antimicrobial resistancerelated death and $M$ tuberculosis the most significant airborne pathogen that is drug-resistant. ${ }^{223}$

Given these projections, addressing tuberculosis drug resistance is essential both for curtailing the global antimicrobial resistance crisis and ending tuberculosis. Although providing universal drug resistance testing and scaling up access to high-quality, tailored treatment for drug-resistant tuberculosis will require substantial funding and commitment, the consequences of not doing so would be enormous, including massive loss of life and trillions of dollars spent as multidrug-resistant tuberculosis increases dramatically. ${ }^{223}$ Furthermore, addressing drug-resistant tuberculosis cannot be separated from scaling up access to diagnosis and treatment of drug-susceptible tuberculosis; if we improve case detection for drug-susceptible tuberculosis without a meaningful change in quality and identification of drug-resistant tuberculosis, we will only increase the selection pressure for this diagnosis.

A modelling analysis commissioned for this report shows the effect of ensuring universal access to drugresistant tuberculosis and second-line therapy in a high drug-resistant tuberculosis country, such as Moldova. ${ }^{140}$ As highlighted in figure 5, optimising access to drugresistant tuberculosis and increasing treatment success rates would lead to a $73 \%$ reduction in tuberculosis mortality and a $43 \%$ reduction in incidence over the next 30 years. With adequate investment in tools, the prospect of definitively addressing the threat of drug-resistant tuberculosis within a generation is credible.

Encouragingly, the rapidly evolving field of drug-resistant tuberculosis diagnostics and the increasing availability of new and repurposed drugs and regimens for treating patients with multidrug-resistant or rifampicin-resistant tuberculosis present opportunities to dramatically improve the epidemic response (appendix p 36). Emerging data suggest that in high-burden settings, more than $90 \%$ of incident multidrug-resistant or rifampicin-resistant tuberculosis results from direct transmission of already resistant tuberculosis bacteria between individuals. ${ }^{73,74}$ As a result, not diagnosing and not effectively treating a substantial proportion of individuals with active disease is a major driver of the epidemic. Barriers to diagnosis and treatment scale-up vary across countries but include the high cost of providing treatment (although data show such costs can decrease dramatically when more individuals are offered access); 224 perceived complexity of treatment regimens; poor programmatic treatment outcomes in most part because of the lengthy and toxic drug regimens that impose enormous burdens on individuals; reliance on centralised and specialised treatment; and lack of political will and commitment. ${ }^{225-228}$

Because most drug-resistant tuberculosis is caused by direct transmission, early diagnosis and initiation of effective therapy, combined with effective preventive therapy for close contacts should be key priorities in preventing this condition. ${ }^{223,229}$ Furthermore, access to new, better tolerated tuberculosis drugs should be prioritised. Policies that spare these drugs for use as last resort options are likely to drive increased drug resistance and are neither scientifically sound nor person-centred. ${ }^{230}$ Rather, strategies for implementing new tuberculosis regimens need to consider the factors that led key first-line drugs to acquire resistance in the past. Such factors include varying individual pharmacokinetics, comorbidities (particularly those that might affect drug absorption, such as HIV), poor drug quality, inadequate dosing, weak supply chains and inadequate prescribing, and selective treatment adherence. ${ }^{231-236}$ Weak health systems that offer limited support for patients and their families contribute to many of these factors, emphasising the importance of strengthening health systems to help respond to the drug-resistant tuberculosis epidemic and provide more person-centred care. ${ }^{237-239}$ Because tuberculosis drug resistance emerges spontaneously and can be selected for during treatment, ${ }^{240}$ using standard combination regimens in patients with undiagnosed drug resistance probably will contribute to further resistance acquisition, in addition to poor patient outcomes. ${ }^{241-246}$ Robust stewardship mechanisms, especially in the private sector, such as that recently described for a large private hospital in India, are crucial in this regard..$^{24}$

\section{Increasing universal access to rapid drug susceptibility testing}

Given the clear requirements to find and treat all individuals with drug-resistant tuberculosis and to prevent the emergence of further resistance, universal drug sensitivity testing (DST; at least to rifampicin) with 
access to second-line treatment is a key recommendation of this Commission. Prompt use of molecular DST for patients who do not respond to first-line therapy should also be implemented to obviate the practice of standardised retreatment with a regimen that only includes one additional drug and is highly likely to contribute to resistance amplification, in addition to poor patient outcomes.

Until relatively recently, diagnosis of drug-resistant tuberculosis relied on tuberculosis culture, with consequent long delays and the need for specialised laboratories. ${ }^{248}$ Because drug-resistant tuberculosis results from the presence of resistance-conferring mutations in the bacterial genome, new tests, such as the Xpert MTB/ RIF test ${ }^{249}$ and line probe assays, ${ }^{250}$ rely on identifying mutations known to infer drug resistance. These more rapid tests have shortened the time required to receive results from months to hours, ${ }^{249,251}$ consequently reducing how long it takes to initiate treatment across a range of settings, ${ }^{252,253}$ and they are being used at scale in some countries. Newer versions of these and related tests, including whole genome sequencing, are expected to expand the range of drugs that can be tested and reduce reliance on specialised laboratories. ${ }^{254-256}$ A pipeline of candidate point-of-care diagnostics, implemented at the same time as an initial health-care visit, have the potential to substantially improve case detection and reduce losses along complicated diagnostic and care cascades. ${ }^{5,257,258}$

\section{Improving drug-resistant tuberculosis treatment}

The high multidrug-resistant or rifampicin-resistant tuberculosis burden and poor patient outcomes highlight the need for safe and effective, less toxic, shorter, and less costly treatment regimens for these populations..$^{67,259-262}$ Encouragingly, two new drugs (bedaquiline and delamanid) are available for use in the treatment of multidrug-resistant or rifampicin-resistant tuberculosis. ${ }^{263-265}$ These drugs, along with drugs repurposed for tuberculosis (including linezolid and clofazimine) and pretomanid (a similar drug to delamanid), are included in a range of new, shorter, all-oral regimens being tested in clinical trials for multidrug-resistant or rifampicin-resistant tuberculosis. ${ }^{266}$ Results from most of these trials, however, are not expected for several years. ${ }^{267}$ In the meantime, these new and repurposed drugs have been increasingly used programmatically. Data from South Africa suggest dramatic improvements in mortality and reductions in treatment ineffectiveness among more than 3000 patients treated with bedaquiline. ${ }^{268}$ As a direct result, South Africa announced in 2018 the implementation of an injectable-free, bedaquiline-containing treatment for all patients with rifampicin-resistant tuberculosis. ${ }^{269}$

The South African data, complemented by a large individual patient-level meta-analysis on multidrugresistant tuberculosis, have contributed to new WHO guidance prioritising the use of bedaquiline and linezolid. ${ }^{270}$ To our knowledge, there is insufficient data to support similar prioritisation for delamanid. Increasing the use of these new and repurposed drugs would remove reliance on some of the more toxic and less effective drugs, including the second-line injectable drugs, which are associated with irreversible hearing loss in up to $50 \%$ of individuals who receive them. ${ }^{271}$ It also would help relieve the burden on the health-care system to deliver the daily injections. ${ }^{272}$ However, uptake of new drugs based on previous WHO guidance has been restricted, despite a US Agency for International Development (USAID) and Janssen Pharmaceuticals (Beerse, Belgium) donation programme in many countries. ${ }^{273}$ Barriers include drug costs, difficulties in individual country regulatory approval and drug procurement, and absence of high level national government support. ${ }^{274}$ Moving forward, these barriers must be overcome. Tuberculosis programmes also need to be continuously evolving, to ensure that national guidelines and clinical practice reflects the best available evidence. Civil society organisations have an essential role to play ensuring that this is the case.

Additionally, a more individualised approach to drugresistant tuberculosis treatment-one that encompasses access to all second-line drugs and is guided by more extensive DST through whole genome sequencingwould enable individuals with this condition to receive the best chance of cure, while limiting both the unnecessary use of toxic drugs and resistance amplification. ${ }^{275}$ Such an approach would need to be supported by implementation research to guide its integration into existing tuberculosis programmes and the health system as a whole, in addition to pharmacovigilance systems. ${ }^{267,276,27}$ While full treatment individualisation might not be feasible in all settings, more stratified approaches that takes into account local drug resistance profiles are potentially feasible. ${ }^{278}$

Given the arduous nature of available tuberculosis treatment regimens (including toxicity and length), as well as socioeconomic challenges, many patients withdraw from treatment before completing the full course which is globally reported as 15\% in the 2014 cohort reported to WHO, and ranging between $1 \%$ and $56 \%$ in individual studies, with a trend to increase as more patients are treated in a particular setting. ${ }^{67,279}$ These data emphasise the need for more person-centred and family-centred approaches that ensure health systems are optimally aligned with the needs of the populations affected by drug-resistant tuberculosis. Although the emphasis has been on improving adherence and reducing catastrophic costs, a personcentred model of care also includes ensuring that people with possible drug-resistant tuberculosis (and those supporting them) are fully informed about, and included in, therapeutic decisions. Such models must tackle active discrimination within the health system and in other sectors. Person-centred care also includes providing treatment closer to where patients live and 
initially seek care (ie, community-based and decentralised treatment centres as much as possible). ${ }^{280}$ Full implementation of such a decentralised approach requires considerable upgrading of the capacity of peripheral facilities to manage patients with a complex resistance profile, who require indvidualised therapy. Such facilities should be supported by easy, routine communication with treatment initiation centres and expert providers. Although a country or region might often have many drug-resistant tuberculosis cases in the aggregate, peripheral facilities might have very few if any patients with multidrug-resistant tuberculosis at any given time. Thus, experience is lacking, and decentralisation needs to occur concurrently with close support from experts, even if those experts are accessed remotely.

\section{Preventing resistance acquisition}

Although diagnosis and prompt treatment are central to tackling the tuberculosis epidemic, minimising the risk of further resistance acquisition, both to existing first-line and second-line drugs and new drugs, is also paramount. Mitigating the risk of drug-resistant tuberculosis transmission includes addressing the drivers of tuberculosis drug resistance through programmatic quality improvement, but also avoiding the use of standardised regimens in the absence of DST whenever possible. Finally, antibiotic stewardship entails ensuring that new drugs are used in tailored, effective multidrug regimens for all patients with drug-resistant tuberculosis, not just those with limited therapeutic options. Such use also needs to be supported by expanded tuberculosis drugresistance surveillance (to replace intermittent, expensive drug-resistant tuberculosis surveys).

As with drug-susceptible tuberculosis, treatment of latent drug-resistant tuberculosis might substantially affect the epidemic in the long term. At least two trials are evaluating different prevention regimens for individuals in close contact with patients with multidrugresistant or rifampicin-resistant tuberculosis. ${ }^{266}$ In addition, WHO released a conditional recommendation in 2018 supporting the use of individualised preventive treatment for contacts of patients with these conditions who are at high risk of progressing to disease. ${ }^{281}$ Given the high morbidity and mortality associated with drug resistant tuberculosis, preventive treatment of these high-risk contacts, including children and people living with HIV, is a priority.

Increasing drug-resistant tuberculosis as a global health and economic security threat-implications for donor financing

The cost of treatment for multidrug-resistant or rifampicin-resistant tuberculosis ranges from estimates of US\$ 1218 in low-income countries to US\$ 83365 in HICs. ${ }^{282}$ The high cost has been a great barrier to scaling up treatment to date. The Stop TB Partnership estimated that, in 2017, US\$2 billion was required to fund drugresistant tuberculosis care; it is expected to increase to US $\$ 3.6$ billion by $2020 .{ }^{67}$ This amount of funding is unlikely to be sustainable for many high multidrugresistant or rifampicin-resistant tuberculosis burden countries; the BRICS countries (Brazil, Russia, India, China, and South Africa) are notable exceptions. As a result, funding to support drug-resistant programme implementation will probably be required from international sources, even in countries with the capacity to fund their own drug-susceptible tuberculosis programmes. The existing and future projected economic costs associated with drug-resistant tuberculosis provides a compelling rationale to justify increased donor financing, even in middle-income countries transitioning out of donor eligibility. Furthermore, investments to strengthen the capacity of high-burden countries to prevent, detect, and respond to drug-resistant tuberculosis will deliver important both global health security dividends. ${ }^{283,284}$

\section{Section 2: investing in tuberculosis research and development}

Despite resulting in more than 1 billion deaths during the last two centuries, ${ }^{285}$ tuberculosis remains poorly understood. Although we can, and must, do more to broadly implement available tuberculosis control tools and strategies, achieving an end to the epidemic will require answering fundamental questions about tuberculosis and developing new biomedical tools to accelerate our progress toward that goal. ${ }^{286}$ The urgency of boosting our investment in research and development to enable these transformative advances demands that governments and their partners in HICs and middleincome countries commit now to sustained, increased funding of these efforts. The UNHLM underscored the crucial role accelerating tuberculosis research and development has and will continue to have in achieving an end to the tuberculosis pandemic. Building on that call to action, in this Commission we highlight research and development priorities and provide an economic rationale for why investment in these priorities is crucial to success.

\section{Biomedical research priorities}

Future successes in developing new diagnostics, therapeutics, and vaccines for tuberculosis will require an improved understanding of the pathogenesis of the disease. In this regard, a key basic scientific priority is identifying the correlates of risk for progression to disease. An intensified search for biomarkers associated with protection from disease, ${ }^{287}$ as well as the development of better animal models, are among other priorities. Large gaps also exist in understanding tuberculosis pathogenesis and the host immune response, especially in children ${ }^{288}$ (panel 4) and in individuals coinfected with HIV. ${ }^{292}$ Nonetheless, promising preclinical efforts exist that must 
be substantially expanded. These efforts include using computational modelling to better understand complex biological interactions between pathogen and host, ${ }^{293}$ high-throughput host genomic screening to identify RNA signatures associated with the risk for disease, ${ }^{294}$ and improved animal models of tuberculosis latency. ${ }^{295}$

To accelerate the development pipelines for diagnostics, therapeutics, and chemopreventive strategies and vaccines, it is imperative to develop an integrated research strategy and agenda to close cross-cutting gaps in tuberculosis research and development (figure 7, appendix p 48). This Commission outlined key research priorities, including those published recently in the US National Institute of Allergy and Infectious Diseases (NIAID) Plan for TB Research. This Plan and similar multipronged, multidisciplinary efforts are essential to substantially advance research and development and end tuberculosis. ${ }^{296,297}$

\section{Diagnostics}

With 3.6 million people estimated to have undiagnosed or unreported tuberculosis, including an estimated 558000 people with undiagnosed, drug-resistant tuberculosis, ${ }^{5}$ the importance of having rapid and accurate diagnostics at entry into tuberculosis care cannot be overstated. Early, accurate diagnosis together with drug susceptibility testing at the time of diagnosis is key to breaking the cycle of transmission, enabling patients to be quickly started on effective treatment. Investments in research and development for diagnostics have led to the progressive introduction of six new diagnostic tools since 2005. These tools have helped overcome major barriers in identifying drug-susceptible and drug-resistant forms of $M$ tuberculosis, including cost, complexity, slow time-to-result, and low accuracy. ${ }^{298}$ An additional 45 candidates are in the tuberculosis diagnostic pipeline. ${ }^{299}$ Unfortunately, many of these tests are molecular technologies that are unlikely to meet the three most important needs of high-burden LMICs.

For high-burden, low-resource settings, the first priority is an easy-to-use, low-cost, non-sputum-based ${ }^{300}$ rapid diagnostic test that can identify individuals with active disease and can be incorporated into active case-finding strategies or used in primary care facilities (appendix p 28). Modelling has shown that a triage test, implemented at the community level and used in combination with a confirmatory test (eg, GeneXpert), could close case detection gaps and reduce incidence by $19 \%$ and mortality by $37 \%$ over 10 years. ${ }^{301}$ The second priority is rapid tests for drug-resistance that would help direct patients to appropriate treatments and safeguard medicines against antimicrobial resistance. ${ }^{302,303}$ Priority three is an incipient tuberculosis in-vitro diagnostic to identify individuals at high risk of progression from latent infection to active disease. This in-vitro diagnostic would enable targeted preventative treatment in communities as a prerequisite
Panel 4: Tuberculosis in children

Tuberculosis is among the top ten causes of death in children under age 5 years, despite being both preventable and treatable. ${ }^{6.94}$ Furthermore, recent modelling suggests that of the nearly 70 million children estimated to be silently infected with Mycobacterium tuberculosis, 1 million develop tuberculosis disease each year; adolescents in particular provide a seedbed for future active disease. Of the 1 million children, only one-third are diagnosed and treated and one-quarter die. ${ }^{6.95}$ The burden of drug-resistant tuberculosis in children is also substantial and yet infrequently diagnosed and appropriately treated..$^{96,97}$

Tuberculosis in children was largely ignored until the past decade, when an increasing number of National Tuberculosis Programmes (NTPs) began developing paediatric guidelines and including children in tuberculosis control strategies. In 2015, the WHO End TB Strategy included children with an emphasis on prevention..$^{98}$ The UN High-Level Meeting includes specific targets to find and treat 3.5 million children suffering from tuberculosis and provide preventive therapy to 4 million more children until 2022.

\section{What can be done now to address tuberculosis in children?}

The need for new tools to address tuberculosis in children is crucial, particularly accurate, point-of-care diagnostics to detect both disease and infection; shorter, more efficacious treatment regimens; and a better vaccine. However, in the meantime, ramping up the use of available tools now could dramatically reduce the burden of tuberculosis in children (appendix $\mathrm{p} 42$ ). Strategies and tools to prevent tuberculosis in children are underused (eg, neonatal BCG coverage, screening of all childhood household tuberculosis contacts, tests for infection, and short regimens for preventive therapy) often simply because of a lack of availability. Moreover, infection control measures to protect children from infection in high-risk settings are implemented only rarely. Effective implementation of existing tools will require integrating other health services, especially those for maternal and child health. ${ }^{99}$ Health service delivery for the detection and treatment of tuberculosis in children can be successfully decentralised and strengthened, ${ }^{289}$ child-friendly formulations for the treatment of infection and disease are available for young children, and the opportunity for the effective implementation of preventive therapy is unprecedented. ${ }^{100}$ Tuberculosis screening and management can also be integrated into other services such as perinatal care, HIV clinics, or nutritional rehabilitation centres. ${ }^{101,290,291}$

Although we must fully implement what we know works, increasing research on how to improve strategies to prevent, diagnose, and treat tuberculosis in children must also be a priority. A substantial increase in investment and sustained advocacy will be needed, but the return will be substantially more children alive and living tuberculosis-free. Childhood tuberculosis has received increased attention in the past decade, but tuberculosis still causes substantial mortality and morbidity among this age group ( $<5$ years). Although new diagnostics, treatments, and vaccines would help manage the paediatric tuberculosis epidemic, what we can and should do now is to integrate childhood tuberculosis management into other health services, implement existing interventions to their fullest, and increase our investment in research and development to save more young lives in years to come.

to tuberculosis elimination in the absence of an effective vaccine.

Achieving priority one requires identifying a suitable host and microbial biomarkers and biosignatures (primarily antigen, antibody, or a volatile organic compound). Several promising diagnostic biomarker combinations have been identified that are undergoing validation or being transferred to point-of-care platforms. ${ }^{304,305}$ If successful, a triage test could be introduced by 2020; however, given high candidate failure rates and 


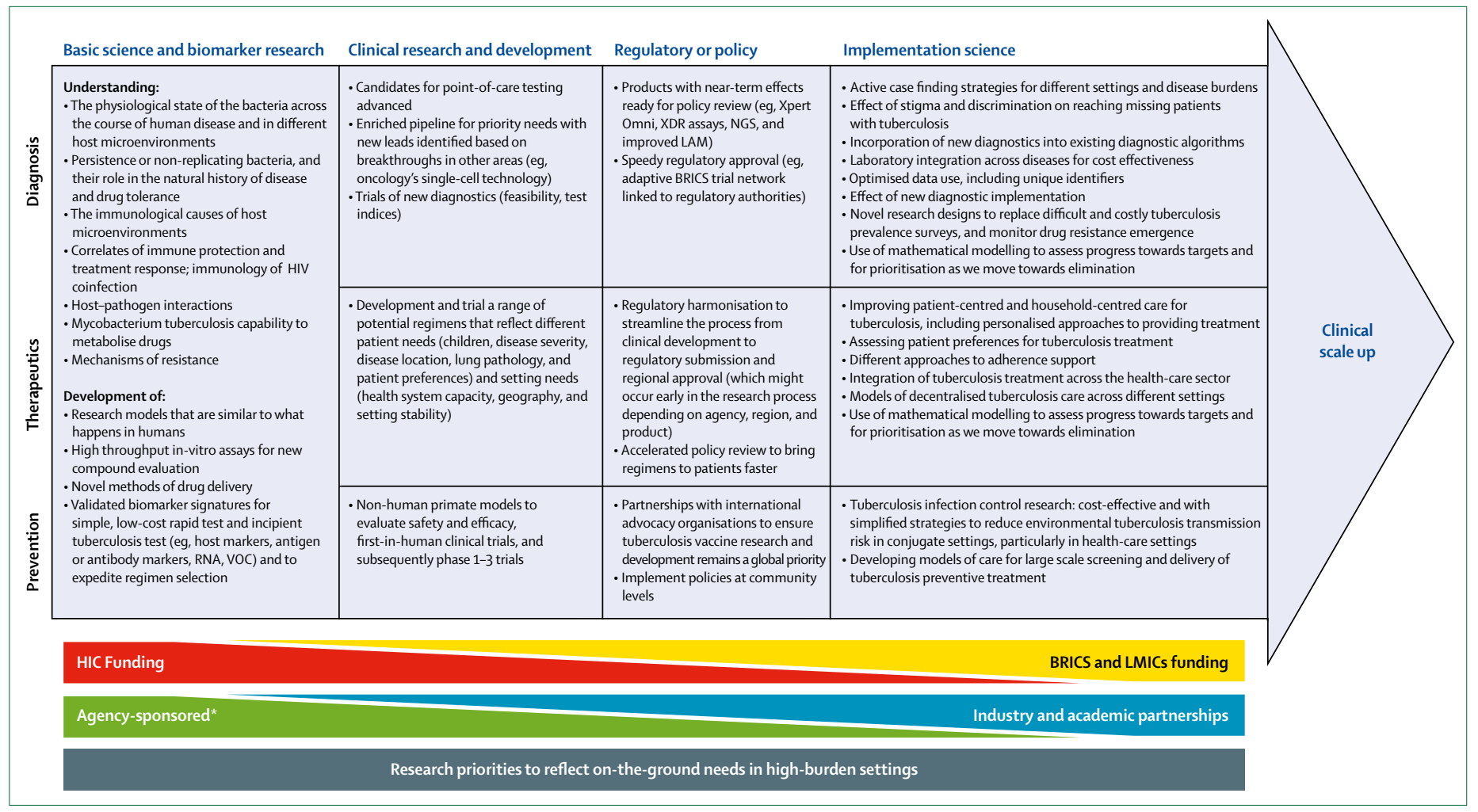

Figure 7: Framing priorities along the tuberculosis research and development pipeline

BRICS=Brazil, Russia, India, China, South Africa. HIC=high-income countries. LAM=lipoarabinomannan. LMICs=low-income and middle-income countries. NGS=next-generation sequencing. $R \& D=$ research and development. VOC=volatile organic compounds. XDR=extensively drug-resistant. *Agency-sponsored (eg, US National Institutes of Health, UK Wellcome Trust).

few priority one candidates in the biomarker pipeline, additional funding is needed to enrich the pipeline. Expansion of the set of tools for drug susceptibility testing is underway for existing molecular platforms, and next-generation sequencing tools have promising results; nevertheless, further translational work is required to make them affordable and deployable in high-burden countries. ${ }^{306,307}$ Similar to the triage test, a breakthrough in biomarker discovery is necessary to diversify the incipient test pipeline, which is sparsely populated. ${ }^{308}$

\section{Therapeutics}

Development of markedly improved therapeutics could rapidly accelerate efforts towards ending tuberculosis. The principal desired characteristics are shorter, nontoxic, patient-friendly treatment regimens that can be implemented widely. ${ }^{309}$ Preferably, the individual components of improved therapies should focus on either novel targets or targets that do not have cross resistance with available drugs. Since approximately 1 million new tuberculosis cases occur in the paediatric population each year, it is also crucial that new tuberculosis therapeutics are formulated to be appropriate and effective for children, as well as for adults. $^{310}$
Developing novel, safer, shorter, and simpler regimens will have to overcome many challenges. The existing drug regimens to treat drug-susceptible tuberculosis are remarkably effective, largely non-toxic, and very inexpensive, although they result in a low threshold to develop drug resistance. New drugs are unlikely to be tested individually but instead added to existing regimens and tested for non-inferiority and safety rather than superiority. As a consequence, many of the newer drugs are being tested on patients with drug-resistant tuberculosis, for which the effectiveness of the available regimens is limited and smaller trials in a defined target population are feasible. Two major challenges in developing novel, safer, shorter, and simpler regimens are the research costs of preclinical development and phase 1 and 2 clinical trials, and the lack of reliable, validated biomarkers that can be used to predict the duration of therapy necessary to cure virtually all patients treated with a given therapy. ${ }^{311}$ The findings of three recent phase 3 trials, ${ }^{312-314}$ which did not shorten therapy for drug-susceptible tuberculosis despite promising phase 2 results, clearly show how the absence of predictive biomarkers constrains clinical research. This issue is particularly problematic because of their complexity and long duration, the cost of late-stage clinical trials of novel tuberculosis regimens is high. 
During the past decade, remarkable progress has been made in the search for new tuberculosis drugs and therapeutic regimens. In the early 2000s, no new drug candidates existed to treat latent tuberculosis; the pipeline currently has more than 30 compounds (although few are new chemical entities), including several drugs in latestage product development (appendix p 42). Two novel drugs have received conditional regulatory approval by WHO. ${ }^{282}$ Because of the pipeline growth, it is now feasible to investigate novel combinations of drugs and new therapeutic regimens. New regimens in ongoing phase 2 and 3 clinical trials show considerable promise and might enable much shorter durations of treatment-even for the most resistant forms of extensively drug-resistant tuberculosis - than what is recommended. ${ }^{315}$ Furthermore, a 2-month universal regimen, active against all forms of tuberculosis, might be possible within the next decade. This regimen would offer the potential to shorten and simplify treatment strategies and drug-susceptibility testing needs, ${ }^{316}$ and should be a high funding priority in the next decade. The potential utility of a pan-tuberculosis regimen must be considered together with person-centred approaches to treatment, and tailored to pharmacogenetics, comorbidities, and drug coadministration, as well as the risk of new forms of resistance. ${ }^{317}$ A diversified portfolio of therapeutic products offers the best hope for long-term success; however, substantial investment in the short-tomedium term is needed to guarantee those products reach the market.

\section{Vaccines and chemopreventive strategies}

Before the antibiotic era, evidence existed to indicate that remarkable protection against tuberculosis could be produced by latent infection, and that BCG was protective in some populations but not others. ${ }^{318}$ Nevertheless, BCG remains the only available vaccine, despite having been developed over 100 years ago, having variable effectiveness in preventing adult pulmonary tuberculosis, and not being recommended for children with HIV. Although compelling evidence from models shows that a vaccine with $60 \%$ efficacy could avert 70 million tuberculosis cases within 25 years if given to only $20 \%$ of at-risk adults, ${ }^{319}$ progress towards developing viable vaccines has been hindered by numerous scientific and funding challenges. In contrast to drugs, vaccines are given to healthy people to prevent illness. Thus, the stringency in being certain that candidate tuberculosis vaccines are as safe as possible represents a high bar. Also, because many individuals who will never be infected have to be vaccinated to show protection in a smaller group infected with $M$ tuberculosis, trials require large populations and access to sophisticated laboratories.

14 candidate vaccines that have shown some degree of protection against tuberculosis in animal models are being tested in ongoing human clinical trials. ${ }^{311}$ Some are live recombinant vaccines (eg, BCG with added antigens and genes to elicit strong immune responses, or genetically attenuated $M$ tuberculosis); others are live virus vectors expressing multiple antigens of tuberculosis to provide long-lasting immunity (eg, recombinant cytomegalovirus vectors expressing tuberculosis antigens). ${ }^{319}$ Only two phase 3 preventive tuberculosis vaccine studies have been published, one using an inactivated whole-cell mycobacterial vaccine (M obteneuse) reporting more than $40 \%$ protection in adults ${ }^{320}$ and the other evaluating the modified vaccinia Ankara virus expressing antigen 85A (MVA85A) to boost the effectiveness of the BCG vaccine in infants, which did not show protection. ${ }^{321,322}$

BCG vaccine is routinely recommended for newborns in tuberculosis endemic countries as it has an important protective role against tuberculosis-related morbidity and mortality in infants and young children. In 2018, evidence has shown that BCG provides a limited degree of protection against infection. However, preventing tuberculosis disease and infection in young children will have a limited effect on the tuberculosis pandemic as tuberculosis in this age group makes minimal contribution to transmission in the community. Two new phase $2 \mathrm{~b}$ trials offer new promise for vaccines against tuberculosis. ${ }^{323}$ Revaccination with BCG of South African adolescents, who received BCG as infants and were without evidence of infection with $M$ tuberculosis (Quantiferon-negative), provided protection against persistence of tuberculosis infection in $45 \%$ of patients who had evidence of recent infection following revaccination. ${ }^{324}$ A new subunit tuberculosis vaccine, with two $M$ tuberculosis antigens in an adjuvant that has been effective in vaccines against herpes zoster and malaria, M72AS01E tested in several thousand adolescents (1786 in the vaccine group and 1787 in the placebo group, 18-50 years, HIV-uninfected, and with confirmed latent tuberculosis infection) in three subSaharan countries, showed protection in $54 \%$ of the patients and, notably, protection in $87 \%$ of those younger than 25 years. ${ }^{325}$ These results emphasise the importance of further clinical trials and suggest the potential of targeting vaccines to young adolescents because protecting against tuberculosis infection and disease in older adolescents and young adults will have a much greater effect on tuberculosis control than protecting young children. There is an ongoing need to continue to search for correlates of protection in human trials, which could shorten the time and expense of future trials.

These encouraging results need to be validated and extended, particularly in different geographic situations, but despite challenges, the scientific prospects for developing a safe and effective vaccine to prevent tuberculosis are promising; an increased focus on earlystage research has led to a robust pipeline and new technologies, which are providing unprecedented scientific opportunities. ${ }^{326}$ Vaccines represent the most cost-effective intervention to prevent disease and death. In the case of tuberculosis, long-term and sustained 


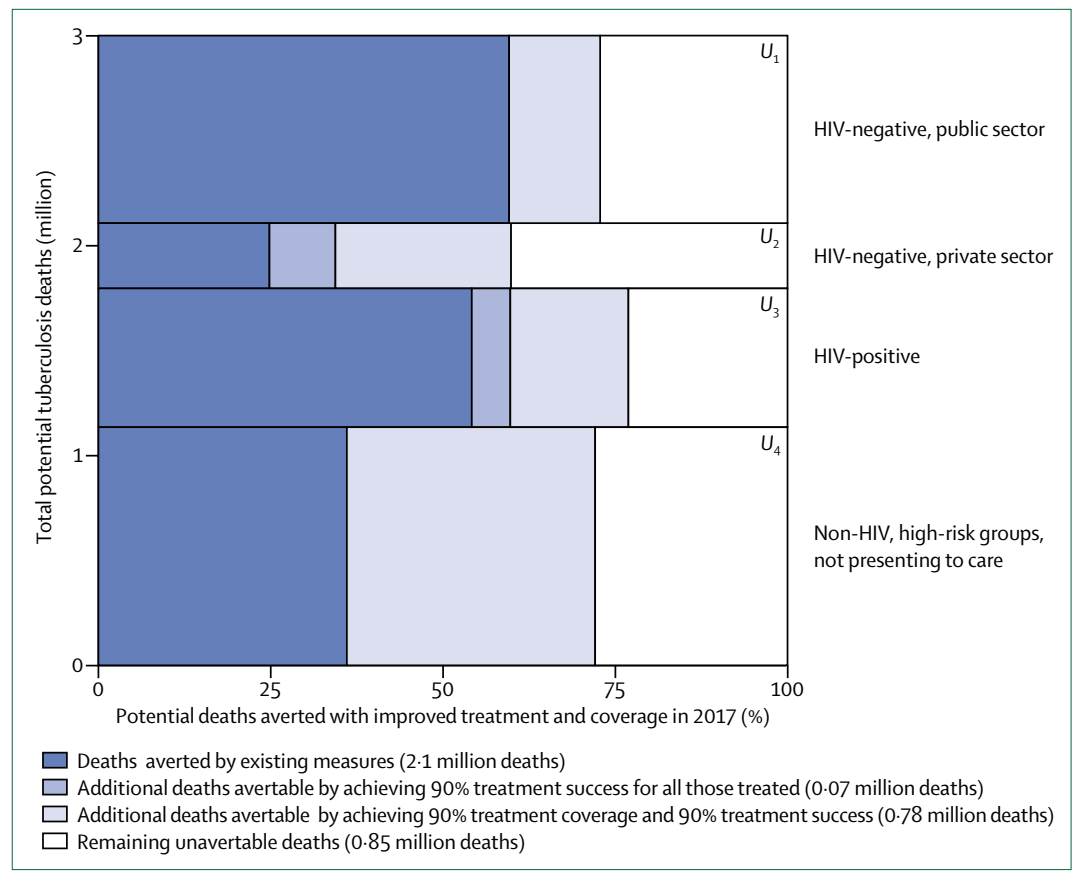

Figure 8: Total potential deaths (averted, unaverted, and unavertable) from tuberculosis, across different populations or risk groups globally (2017)

Increased case finding will lead to a reduction in unaverted deaths, but achieving $90 \%$ case detection and diagnosis and $90 \%$ treatment coverage will still leave a substantial burden of unavertable deaths ( 0.85 million people). New tools and control strategies are needed to prevent these unavertable deaths. These estimates do not include the effect of strategies to avert tuberculosis deaths in low-burden settings, such as Europe and the Americas. Furthermore, estimates do not include drug-resistant tuberculosis coverage, neither take into account the potential effect of prevention interventions, but rather provide a static presentation of the effect of increasing case detection, diagnosis strategies, and treatment success across different population groups. Deaths averted were calculated based on approach adapted from Glaziou and colleagues. ${ }^{333}$ Full set of assumptions and explanatory calculations are in appendix $\mathrm{p} 41$. U=unavertable.

investments will be necessary to build on these promising results, but the returns even from a partially effective vaccine would be very great.

\section{Population, policy, and implementation research priorities}

Progress towards ending tuberculosis has been limited because existing tools have been ineffectively implemented and the control strategies used are outdated. Greater national and global investments in population, policy, and implementation research capacity will be required to enable the scaling of effective approaches. ${ }^{117}$ In particular, implementation research is needed to understand how to improve care cascades (ie, find patients early, evaluate them quickly, and provide effective treatment that results in a cure). Population research to characterise the factors that drive tuberculosis transmission within families and communities, particularly in high-burden settings, is also crucial for developing innovative strategies to interrupt $M$ tuberculosis transmission. ${ }^{45}$ While research on sensitive, inexpensive point-of-care diagnostic tests continue, active screening strategies could be implemented with existing technologies, including automated radiography screening (ie, interpreted by a software rather than a radiologist) in contacts and high risk groups in high burden countries, followed by culture or Xpert testing diagnosis, on the basis of the strong evidence from surveys showing that $20-30 \%$ of tuberculosis cases globally are asymptomatic., ${ }^{5,167,197}$

To optimise treatment outcomes, differentiated strategies for providing person-centred care and supporting treatment adherence must be developed in concert with the creation of new therapeutic regimens. ${ }^{131,327,328}$ Likewise, research is necessary to determine the most efficient and cost-effective tuberculosis prevention therapies. The potential of digital technology to overcome weak health system infrastructures, enhance tuberculosis programme quality, and improve disease surveillance remains largely untapped. Although numerous disparate pilot studies have been done evaluating information technology, electronic health, and connectivity solutions, ${ }^{327,329-332}$ future studies should be guided by a comprehensive research agenda supported by a commitment from countries and funders to translate evidence to action at scale.

Furthermore, mechanisms must be identified and implemented to strengthen the infrastructure and capacity of countries to accommodate-in terms of both speed and scale-innovations, as well as to rapidly translate research findings into policy. ${ }^{28}$ For instance, the Initiative for Providing Affordable and Quality Tuberculosis Testsprovides a proven model for incentivising the uptake of new diagnostics among private sector providers in India; however, it has yet to be translated into a replicable model and implemented in other countries. In part, this problem reflects the need for improved implementation research capacity in LMICs to realise the benefits of investing in tuberculosis research and development. ${ }^{4}$ The role of transnational research networks to build such infrastructure and capacity is essential.

\section{The cost of inaction in research and development}

The human costs of not developing and implementing new and improved interventions is unacceptably high. Even in the WHO's best case scenario in which treatment coverage was extended to $90 \%$ of people with tuberculosis and $90 \%$ were successfully cured (substantially higher than what global estimates indicate), ${ }^{5}$ we estimate that there will be nearly 1 million unavertable deaths with the available technologies (figure 8). To achieve these $90 \%-90 \%$ goals would require unprecedented case finding, treatment completion and prevention, and yet would still be inadequate to reduce global tuberculosis numbers enough to achieve End TB targets, underscoring the important need to close gaps with scientific discovery and programmatic innovation.

The potential economic value of new tools is shown by modelling analysis in three different country-settings, India, Kenya, and Moldova (figure 9), leveraging an approach with which the value of lives lost prematurely was derived using the value of life statistical estimates (appendix pp 43, 44)..$^{11,334,335}$ Optimal implementation of existing evidence-based strategies to improve the care 
continuum for active tuberculosis in each of those countries will still leave millions of deaths unaverted over the next 30 years. The value of the loss associated with tuberculosis mortality is, on average, $\$ 32$ billion per year in India, $\$ 2.7$ billion in Kenya, and $\$ 35$ million in Moldova. However, these numbers are likely to be underestimates since they arise from an arguably ambitious scenario, of reducing losses in the care cascade to $10 \%$ and delays by $25 \%$; and they do not account for opportunity costs associated with unaverted disease that does not result in death, nor the financial burden placed on the health system associated with this unaverted disease burden. India's recent National Strategic Plan, for example, calls for increased uptake of preventive therapy, as well as addressing risk factors for tuberculosis, such as undernutrition. Even though these interventions would have a big effect on the Indian tuberculosis burden, new tools are needed to drive the burden to zero.

Many reasons explain the gap in investments in tuberculosis research and development. The most obvious is that the highest burden of disease occurs in LMICs, which are not able to afford new expensive tests and drugs. Because of a relatively low prevalence disease compared with other infectious diseases and a high latently infected population, efficacy testing of new tools will require large and lengthy trials. Finally, new tools are only as effective in controlling the disease as are health systems able to implement them, and hence improvements in health systems are crucial. Nonetheless our analysis clearly shows that further tools, particularly tools for primary prevention, will have a large return on investment, to the extent that they prevent these needless tuberculosis deaths. Furthermore, it validates the argument that greater spending in tuberculosis research is likely to bring important economic benefits and have a disproportionately beneficial effect on health outcomes in LMICs. ${ }^{113}$ It also underscores how proposed investments in research and development, estimated to be US $\$ 8.7$ billion over the next 4 years, ${ }^{160}$ represents an excellent return on investment. If new tools were developed that would enable reaching WHO's targets, it is estimated that the return on investment for each US dollar, depending on the value per death-averted and the assumed discount rate, would be US\$16-82. ${ }^{336}$

\section{Reaching global tuberculosis research and development goals}

Despite powerful public health and economic rationales for investing in tuberculosis research and developmentessential for producing breakthrough technologies and strategies to end tuberculosis - a substantial gap in financing remains. There are many reasons for this, including the lack of financial incentives to produce new tools, the cost and duration of clinical trials, and the lack of compelling demand by affected countries. Global funding for tuberculosis product development was US\$ 726 million in 2016, ${ }^{299}$ only one-third of the annual

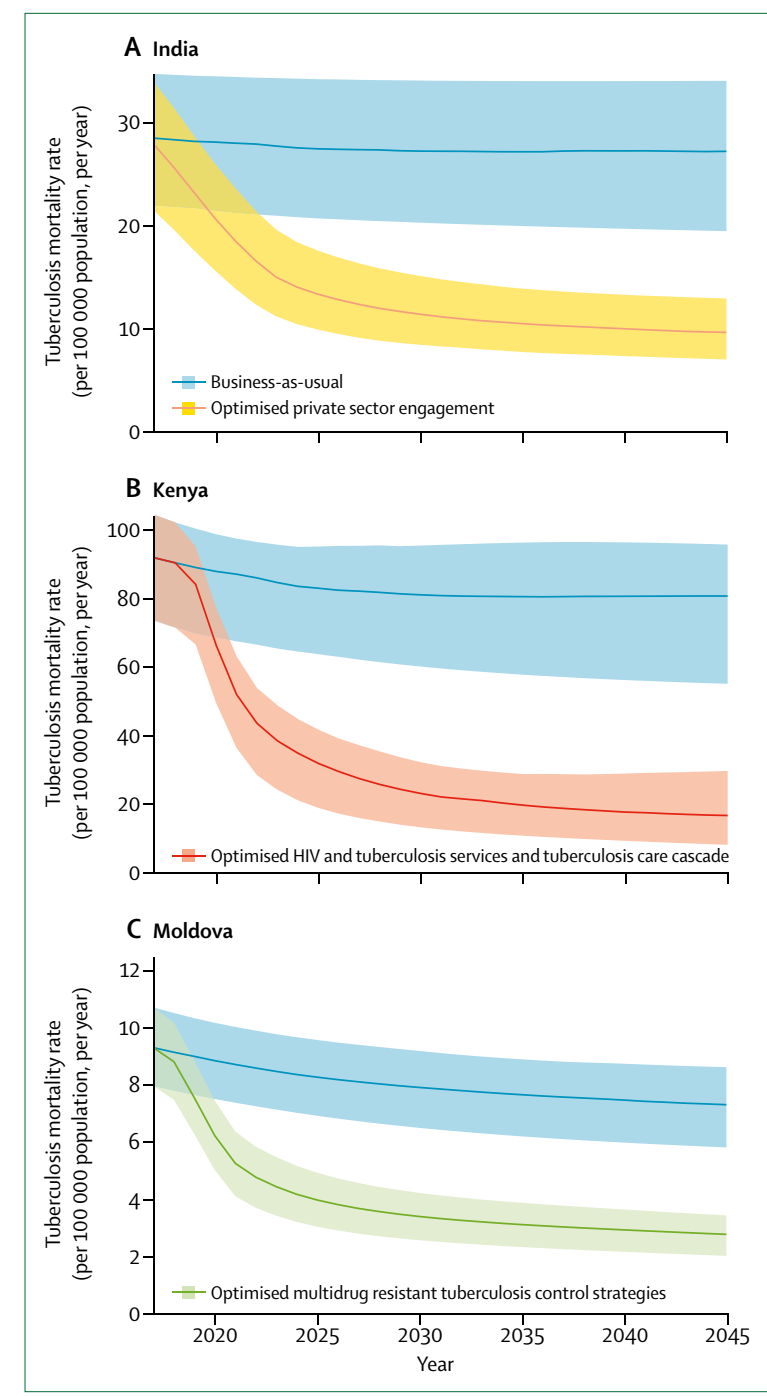

Figure 9: Costs of inaction in tuberculosis research

Curative therapy is the mainstay of tuberculosis control: its maximum effect is realised when patients receive appropriate treatment as soon as possible. The care cascade highlights stages when patients are lost to follow up, in the pathway starting from active disease and ending in treatment completion. To capture the potential effect of curative tools in a simple way, a scenario is shown in which losses in the care cascade are reduced to $10 \%$, and delays are reduced by $25 \%$. Additionally, for Kenya (a high HIV burden setting), we modelled an increase in coverage of isoniazid preventive therapy to $90 \%$ of HIV cases on antiretroviral therapy, as well as an increase of antiretroviral therapy coverage to $90 \%$. Even with such aggressive measures, the figure shows ongoing tuberculosis burden in each of these different settings. Expressed in monetary terms, these ongoing tuberculosis deaths represent a lost value (per year) of US $\$ 31.7$ billion, US $\$ 2.7$ billion and US $\$ 35$ million, for the three country settings shown here (in per-capita terms, US $\$ 24$, US $\$ 55$ and US $\$ 9.8$ per person per year, respectively).

funding called for by the Stop TB Partnership, and far less than is desirable to achieve similar research and development breakthroughs that have characterised HIV research over the past two decades. ${ }^{160}$ Modelling analyses have suggested that current funding might be sufficient to realise some key, near-term successes (eg, a triage test and regimens for drug-resistant tuberculosis based 
on repurposed drugs), but that a multiple of current funding - at least two times, but as high four times the current investment-is needed to enable the development of truly transformative treatments and prevention tools (eg, an incipient tuberculosis test and new vaccines)..$^{337,338}$ Closing the funding gap of at least US\$1.3 billion per year will require HICs to sharply increase their investments in tuberculosis research and development, simultaneously with increased efforts from LMICs, particularly BRICS, as well as the development of creative funding models that enhance industry commitments.

$89 \%$ of the available investment in tuberculosis research and development comes from non-commercial sources (ie, governments and philanthropies). US public agencies alone support $44 \%$ of all tuberculosis-related research globally. ${ }^{299}$ Only a small fraction of the public funding comes from LMICs. ${ }^{339}$ Increasing contributions from LMIC governments so that their total share of tuberculosis research and development matches their share of the global economy (ie, 36.5\%), as has been proposed by a WHO expert group, would generate an additional US\$ 146 million per year, a 26\% increase in total global research and development financing. Given that late-stage clinical trials represent a crucial funding bottleneck, a self-funded BRICS or LMIC clinical trials network, which is focused on bringing innovative tools through the regulatory pipelines, would be another way for high-burden countries to support a greater share of the tuberculosis research and development costs. It would be possible to increase public contributions further if some HICs (or philanthropies) were willing to match increased contributions from LMICs, as Switzerland offered to do to stimulate LMICs to contribute financing for several WHO-selected research and development projects in $2014 .{ }^{340}$ This type of matching grant could increase total funding to US\$861 million per year, representing a $52 \%$ increase (appendix p 45). Matching funding from international donors and high-burden countries could also ensure research is more driven towards the countries' needs and address the issues of countries withholding resources as long as others cover the costs. ${ }^{341}$

Meanwhile, industry investment in research and development for tuberculosis has stagnated, although it has had meaningful increases for other infectious diseases. ${ }^{299}$ Unitaid, through small taxes on international air travel, is an increasingly important source of funding for tuberculosis research and development, providing US\$215 million in 2018 for a variety of innovative research projects. However, more creative models to secure private investment, collaboration, and partnership are needed to close the funding gap. Examples include the TB Drug Accelerator, a collaboration between pharmaceutical companies and research institutions, which has had several early successes in addressing the shortage of new tuberculosis drugs by funding early-stage drug discovery, ${ }^{342}$ and the Global Health Innovative Technology Fund model, a Japanese government funding mechanism that leverages matched funding from industry. ${ }^{343,344}$ Other funding mechanisms including downstream investments or pull strategies (that promise reward for successful product development) have a potential role in funding tuberculosis research and development. ${ }^{336}$ The Life Prize (appendix p 46) offers a novel model to stimulate drug development, rewarding researchers and developers fully and upfront for their investments, thereby delinking the financing of research and development from product prices and sales and promoting access and affordability, as well as appropriate use of resulting products.

Although these various options could represent an important increase, funding will still be far short of the US\$2 billion annual target. This shortage highlights the inescapable conclusion that HICs must contribute more. To ensure the necessary increased investment from HICs, tuberculosis research and development must be understood as an important global public good that will yield substantial economic dividends. Greater investment is also essential to address negative cross-border externalities that tuberculosis, particularly drug-resistant tuberculosis, poses and as central focus of the broader antimicrobial resistance research agenda. Hence, strong advocacy for increased funding to science ministries and researchoriented pharmaceutical companies must occur in tandem with advocacy to international donor agencies.

\section{Section 3: sustainable financing for tuberculosis} Everyone dedicated to achieve an end to tuberculosisaffected countries, donor nations, the private sector, foundations-must redouble their efforts to finance strategies that are working and, more importantly, strategies that have the real potential to make a substantial impact in the coming years. To end tuberculosis, this Commission advocates for substantially more investment in all aspects of tuberculosis programming. Increased domestic resource mobilisation will be especially important, but new models of donor financing that can catalyse domestic investment must also be a priority. Evidence on the cost-effectiveness and benefits of expanded financing for tuberculosis control suggests that such investments will yield a high economic return. ${ }^{345}$

\section{Economic evaluation of tuberculosis control interventions}

In this section we will distil a highly heterogeneous published literature into indicative values of key economic parameters. The section will focus on two such parameters: the cost required to avert a tuberculosis death and estimates of benefit to cost ratios for tuberculosis control efforts. An additional important question is the cost required to meet goals and we provide an approximation that is broadly consistent with this Commission's goal of reducing the global tuberculosis death rate by $90 \%$ compared with 2015 , estimated to be 2 deaths per 100000 population per year. Such estimates of cost are closely bound with questions of 
revenue generation or finance that are dealt with in the finance section of this report. Benefit-to-cost and costeffectiveness ratios in this section will be generated under the same sets of assumptions as are the total cost estimates of the domestic finance section.

The published literature ${ }^{346}$ contains multiple estimates of different indicators of programme effectiveness for different interventions, in different environments, and with different assumptions about how much health system strengthening costs should be included in the cost estimates. The published literature is far less well developed in assessing to whom costs and benefits accrue. The diversity of the literature poses problems for the high-level message objective of this Commission, but at the same time it provides multiple valuable starting points for analysts with different objectives and interests. Such estimates meet the objective of positioning our thinking even though the numbers themselves make no claim to portray any particular set of conditions.

\section{The ratio of benefits-to-costs for tuberculosis control}

Benefits are estimated using methods that are standard in many governments' (and the Organisation for Economic Co-operation and Development [OECD]'s) guidelines for economic evaluation of projects. ${ }^{347}$

The Bill \& Melinda Gates Foundation recently commissioned a so-called reference case analysis to help standardise benefit-cost analyses of projects in LMICs within the broad conceptual framework of the OECD's approach. ${ }^{346}$ This Commission adopts their recommended approach assuming, for illustration, a country with a Purchasing Power Parity (PPP) income of $\$ 5000$ per year. This PPP would be typical of high tuberculosis burden countries. Although many caveats accompany the reference case, its suggested analytic value in a benefitcost analyses for averting a death would be about $\$ 250000$ PPP dollars or perhaps \$70-80 000 exchange rate dollars in a country at that income level. Analyses on this basis suggest that the economic assessment done by the consulting firm KPMG (Amstelveen, Netherlands) and $\mathrm{WHO}^{348}$ that estimated the cost of not responding to the tuberculosis epidemic did not fully capture the value gained from successful tuberculosis interventions. Rather than convey a highly heterogenous range of estimates, we chose instead to rely on recent efforts to aggregate the literature. ${ }^{5,114,286}$ These efforts provide estimates of cost per death averted that are typically implicit in the published analysis rather than reported (table 6). Acknowledging major heterogeneity and uncertainty, it is reasonable to think that the cost per death averted from drug-susceptible tuberculosis would be in the range of US\$700-8000 and US\$ 5000-55000 for drug-resistant tuberculosis. As highlighted in additional figures (appendix pp 48, 49), the uncertainty around these estimates is considerable, reflecting the diversity of settings in which tuberculosis mortality remains substantial.

\begin{tabular}{|c|c|}
\hline & Cost per death averted (US\$) \\
\hline \multicolumn{2}{|l|}{ Global Health $2035^{114}$} \\
\hline Low-income countries & 5000 \\
\hline Lower-middle-income countries & 6000 \\
\hline \multicolumn{2}{|l|}{ Stop TB (Global Plan to End TB) 349} \\
\hline Standard investment scenario (all but OECD countries, 2016-20) & $7000^{*}$ \\
\hline \multicolumn{2}{|l|}{ Estimates provided by WHO Global Tuberculosis report ${ }^{5} \dagger$} \\
\hline Drug-susceptible tuberculosis & 8000 \\
\hline Drug-resistant tuberculosis & 16000 \\
\hline \multicolumn{2}{|l|}{ Estimates provided by Copenhagen consensus } \\
\hline Drug-susceptible tuberculosis ${ }^{346}$ & $700-2000$ \\
\hline Drug-resistant tuberculosis ${ }^{346}$ & $5000-55000$ \\
\hline Drug-susceptible and drug-resistant tuberculosis $\mathrm{s}^{350}$ & 1500 \\
\hline \multicolumn{2}{|c|}{$\begin{array}{l}\text { These estimates are implicit in the sense that they are not provided in the source but instead calculated from information } \\
\text { in the source. } O E C D=\text { Organization for Economic Cooperation and Development. TB=tuberculosis. }{ }^{*} \text { Calculated assuming } \\
\text { one death averted per } 25 \text { disability-adjusted life-years averted. } † \text { When cost-effectiveness numbers were cast as cost per } \\
\text { disability-adjusted life year (DALY) in this Report,they were converted to deaths averted by multiplying by } 25 \text {. The exact } \\
\text { ratio would depend on age of death, the particular set of disability weights chosen and whether the analyst chose to } \\
\text { discount future life years saved. }\end{array}$} \\
\hline
\end{tabular}

Using US\$7000 as an approximation, albeit with inherent uncertainty, of the cost per tuberculosis death averted and \$70-80000 as the per death averted approach to valuation, we estimate a benefit-to-cost ratio for tuberculosis interventions of 10 to 1 . This figure reflects the Stop TB cost estimate ${ }^{349}$ in table 6 for multiintervention programmes required to sharply reduce mortality. It represents a robust estimate of what the global investment required to ensure that countries are on track to achieve the End TB target, hence it can be viewed as an average across the range of required interventions. Other estimates have been higher. ${ }^{34,350}$ Regardless of the method, uncertainty concerning a specific value abounds. Nevertheless, no serious uncertainty attaches to the conclusion that the value of benefits of averting a death from tuberculosis exceeds the value of its costs by more than a factor of 3 to 5 .

Several estimates of cost per tuberculosis death averted (and the associated benefit-to-cost ratio of intervention) come from the Copenhagen Consensus exercises that were sponsored by the Copenhagen Business School (Copenhagen, Denmark) and the Economist. These exercises requested economists representing health and a broad range of other sectors to identify the most attractive interventions within their sectors and to do careful benefit-cost analyses so that expert panels of Nobel Laureates and other eminent economists could critically assess cross-sector development priorities. Two sets of the analyses reported in table 6 were thus critically reviewed by economists outside the health sector. ${ }^{347,350}$ The expert panel of economists for the 2012 Copenhagen Consensus rank ordered 30 attractive investment priorities across sectors. ${ }^{351}$ In terms of the benefit to cost ratio and total potential benefit, tuberculosis treatment ranked number 5 on their list of 30 interventions across all sectors. 


\section{Costs of ending tuberculosis in a generation}

As tuberculosis incidence declines over time, both because of expanded control efforts and (probably) favourable trends in poverty and other risk factor reduction, it is reasonable to project declines in needed expenditure to keep tuberculosis deaths at very low numbers. Initially, if tuberculosis deaths were to be reduced by $90 \%$ from 1.7 million per year to under 200000 per year, the additional expenditure required would be on the order of about US\$10 billion per year (ie, the product of 1.5 million averted deaths per year and a cost of US $\$ 7000$ per death averted).

It would not be possible to scale up within a few years and early investments will yield reduction in cases and costs. However, a plausible cost trajectory for ending tuberculosis in one generation would be an increase in expenditure of about US\$5 billion per year, followed by a reduction to a long-term amount of US\$1 billion to US\$2 billion per year by the early 2040s. This number reflects a reduction in incidence and hence treatment costs that ending tuberculosis mortality will require. This Commission makes no attempt at precision concerning this number in the belief that our basic understanding of the relevant determinants of cost remains highly imperfect: expressing precise numbers is more likely to mislead than inform. That said, these numbers provide a reasonable approximation of the magnitude involved.

\section{Domestic financing for tuberculosis}

We examined the extent to which tuberculosis programmes rely on domestic sources of finance in highburden countries and the influence of domestic financing on the sustainability, efficiency, and equity of tuberculosis funding. In addition, we explored the potential for rapidly increasing domestic financing for tuberculosis until 2023. Finally, we highlight the importance of investing in national tuberculosis programmes and other domestic funding agencies of tuberculosis services to allocate, distribute, and manage domestic tuberculosis resources, recognising that it is essential to develop the capacity to ensure increased financing is spent effectively to end the epidemic.

\section{The pivotal role of sustained domestic financing for tuberculosis}

Improved domestic financing for tuberculosis is one of the success stories in global health over the past two decades. By 2017, 84\% of funding for tuberculosis was from domestic sources. This high proportion reflects a consistent pattern of increased commitment to tuberculosis from high-burden countries. ${ }^{5}$ From 2007-17, global funding for tuberculosis doubled, with much of the increase coming from BRICS. On average, the BRICS have domestically financed $95 \%$ of their public tuberculosis expenditures over the past decade. ${ }^{352}$

Outside of the BRICS, the picture of domestic funding for tuberculosis is complex, reflecting a general scarcity in health sector resourcing and capacity. In 2017, less than half of public funding for tuberculosis in low-income countries came from domestic sources. ${ }^{5}$ Nonetheless, the progress over time is promising; on average, low-income countries doubled their domestic financing of tuberculosis between 2007 and 2017, with a rate of increase similar to that of international tuberculosis funding to their countries. ${ }^{95}$ Not all low-income countries are following this trend, and there is room for improvement: the proportion of the domestic contribution to public tuberculosis expenditure ranges from less than $1-24 \% .{ }^{95}$ Likewise, in lower-middle income countries, the proportion of domestic public funding ranges from $7-88 \%,{ }^{95}$ with the average growth in domestic tuberculosis financing stable until 2013, but doubling since then.

\section{Who provides domestic finance, and how does it get allocated to tuberculosis?}

Public sector tuberculosis expenditures can be divided into those that are allocated through general health service provision and those allocated through national tuberculosis programmes. Although the proportional domestic contribution to overall tuberculosis expenditure is generally high, national tuberculosis programmes-specific expenditure and tuberculosis-specific commodities are more reliant on international finance. In 23 of the 30 highburden countries, these programmes receive more than $80 \%$ of their funding externally, ${ }^{95}$ with the Global Fund being a substantial payer for tuberculosis commodities. This apparent dependency of national tuberculosis programmes on international finance has probably arisen because of the disease-specific allocation of international funds, rather than reflecting an overall lack of domestic commitment. Ministries of Finance inevitably reduce domestic resource allocation to tuberculosis to the extent that they perceive international finance to be available.

Domestic financing for tuberculosis within countries can come from a range of sources. Ultimately, populations and corporate taxes are the main payers, but patients with tuberculosis still face much of the burden in some countries. Despite the policy of free or reimbursed tuberculosis care in most countries, patients can still incur substantial out-of-pocket payments for public tuberculosis services. ${ }^{282}$ Moreover, in several high-burden countries, large proportions of patients seek and receive tuberculosis care in the private sector, paying for their own care and treatment. Subsidising and pooling these private domestic expenditures, an important goal of the broader UHC agenda, will have beneficial consequences in terms of financial risk protection ${ }^{353,354}$ and possibly health outcomes ${ }^{355}$ for those with tuberculosis.

\section{Is the allocation of domestic finance to tuberculosis efficient?}

Although many countries have increased their allocation of public money to tuberculosis, a mismatch remains between funding amount and need, with 
need being defined in terms of the resources required to reach global End TB targets. ${ }^{95}$ From a domestic public finance perspective, however, need is not a sufficient criterion to increase investment. Ministries of Finance will have requests to fund many other development and health interventions that have potentially high returns. Hence, those advocating for increased investment in tuberculosis, both within and external to governments, need to show that investment in tuberculosis has a high return, at the very least compared with other health sector investments. Investments in tuberculosis hence need to be efficient, defined as maximising population health for any given amount of funding.

Increasingly, countries are developing public finance processes that formally assess the return on investment of different health sector interventions, rather than relying on global evidence. These processes are being supported by improved data and understanding of the costs, effectiveness, and long-term effects of the investment in tuberculosis on both health and economic outcomes. ${ }^{356}$ Therefore, supporting these efforts often provides favourable evidence in favour of supporting resource allocation to tuberculosis programmes. In Malawi, for example, an assessment to determine the essential package of health care in 2017 found that seven of the top ten best buys for health sector budget prioritisation were tuberculosis interventions. ${ }^{357}$ This assessment mirrors systematic reviews of return to investment of tuberculosis expenditures across several countries, ${ }^{172}$ supporting the assertion that increasing domestic allocation to tuberculosis can improve the efficiency of the entire health sector.

However, improvement of the efficiency of tuberculosis expenditures is possible through improvements in the delivery and implementation of tuberculosis services, as highlighted in section 1 . In some countries, the division of tuberculosis expenditures on commodities versus general service provision might not be optimal. Improvements in health system strengthening are crucial to ensure that health staff at the front end of tuberculosis service delivery receive the right mix of resources to provide high-quality person-centred tuberculosis services. ${ }^{358}$ Some countries also have higher than average tuberculosis treatment costs, because of he overhospitalisation of patients with tuberculosis, in particular those with drug-resistant tuberculosis. Nonetheless, the decentralisation of drug-resistant tuberculosis care in South Africa exemplifies the substantial additional funding that might be generated by reducing hospitalisation for patients, including those requiring intensive treatment for drug-resistant tuberculosis. ${ }^{359}$ Improved integration of tuberculosis services might also support person-centred care and reduce costs. ${ }^{360}$ Several new tuberculosis technologies, such as shortened regimens, might reduce the costs substantially. More analyses on the efficiency of these different approaches to scale up tuberculosis services is necessary to help guide how countries can spend funding effectively. ${ }^{361}$

\section{Can domestic funding for tuberculosis be substantially increased?}

Generating additional domestic financing for tuberculosis depends on governments' commitment to allocate more funding to tuberculosis; the future potential for efficiency gains; and increases in the overall amount of available public finance. Increases in domestic financing for tuberculosis in the past two decades show that countries with GDP growth might be able to expand their funding of tuberculosis rapidly while reducing tuberculosis incidence. ${ }^{362}$ In addition, the ability to raise domestic finance for tuberculosis from private individuals and firms depends on the system of revenue generation and taxation structures. In the past decade, a range of innovative mechanisms, including earmarked taxation of alcohol and cigarettes, government loan buy downs (ie, in which a third party contributes to loan payment to open up social spending), and the expansion of health insurance coverage, have been explored to improve the financial sustainability of the health sector, with positive consequences for population health. ${ }^{363}$ These mechanisms have yet to provide substantial funding for HIV, ${ }^{364}$ and considerable questions remain as to their feasibility to raise a high amount of funding for tuberculosis.

We did a fiscal space analysis in collaboration with a team at the Department of Global Health, London School of Hygiene and Tropical Medicine, London, UK, and the Institute for Global Health Sciences, University of California, San Francisco, CA, USA. This analysis examined the potential fiscal space and its implications for tuberculosis financing for 28 of the 30 high-burden countries until 2023 (two countries, Zimbabwe and North Korea, excluded because of data scarcity). Fiscal space analyses apply international public financing norms to available fiscal performance to determine the extent to which funding can grow in a way that does not damage overall fiscal stability. The financing sources examined included GDP growth, increasing public revenues, improving allocation to the health sector, improving allocations to tuberculosis, and increasing the efficiency of public tuberculosis service delivery. The researchers found that most high-burden tuberculosis countries can substantially increase public domestic financing of tuberculosis. By 2023, countries such as Bangladesh, Zambia, China, and Indonesia can potentially increase their annual tuberculosis expenditures more than fivetimes, through a combination of optimised resource allocation, revenue generation, and improved resourcing of the health sector (figure 10). In countries like Zambia, increased prioritisation and efficiency of tuberculosis services would enable the greatest resource mobilisation for tuberculosis. In countries such as Bangladesh, China, 


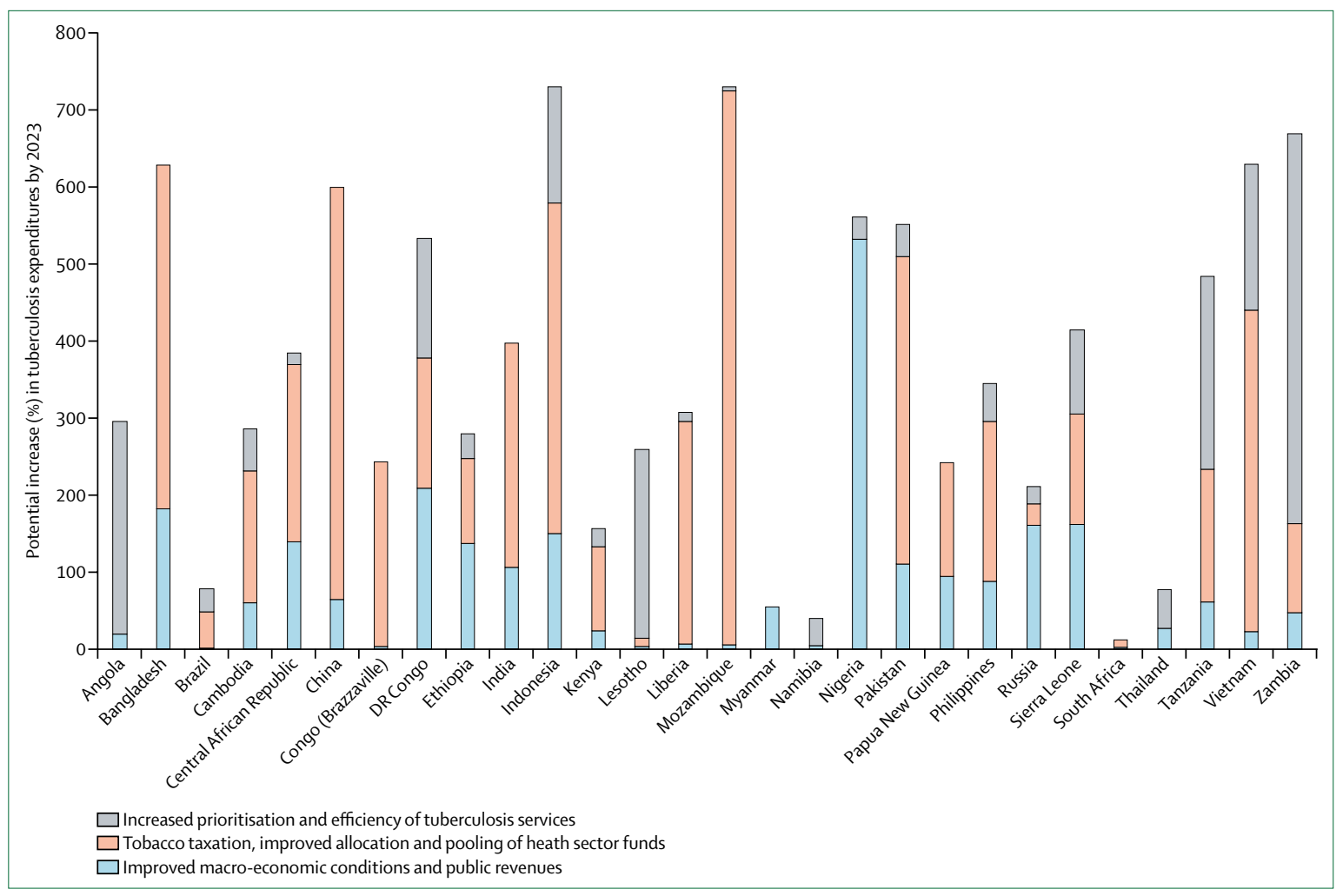

Figure 10: Increase in tuberculosis expenditure by 2023 in 28 high-burden countries

Baseline data on domestic funding for tuberculosis in 2016-17 was from WHO Global Tuberculosis Report ${ }^{95}$ (2017).

and Indonesia governments will need to commit to substantial policy action around revenue raising, such as increasing tobacco taxation for public revenue and the increased pooling of health sector funds. Despite the potential effect of tobacco taxation highlighted in this analysis, we acknowledge the limitations of raising tax in the short term and advocate for optimised resource allocation and improved resourcing of the health sector as the most sustainable means of increasing financing for tuberculosis. Although this report points to the advantages of using proceeds from increased tobacco taxes for health finance, and tuberculosis control more specifically, we acknowledge reasonable arguments for maintaining those proceeds as general revenues. Likewise, we point to a tension between having tobacco taxation mainly as a source of public revenue and having its principal purpose be to reduce smoking through punitive taxes to, ultimately, very low levels. The latter purpose would undermine the revenue generation purpose, perhaps to overall good effect.

\section{Policy implications}

Mobilising domestic resources for tuberculosis will take policy action and commitment across government, including Ministries of Finance and Ministries of Health. Increasing tobacco taxation and allocating those revenues to health is a clear policy action that can support financing tuberculosis elimination and have positive benefits for people with tuberculosis. Increasing domestic public financing for tuberculosis in a manner that protects patients with tuberculosis from catastrophic expenditures is particularly important and serves a broader UHC agenda.

However, it should not be assumed that high level commitment to this broad policy agenda is sufficient. Rapid increases in domestic financing for tuberculosis will require enhanced capacity to allocate and spend resources effectively and transparently to achieve results. A clearly defined accountability framework to ensure commitments made at the UNHLM will be crucial. In addition, national tuberculosis programmes need to strengthen their absorption capacity, otherwise the rate at which additional financing is disbursed in practice might be slow. The experience of HIV shows it is possible to rapidly strengthen programmes, but that strong systems are required to ensure efficiency and maximise health outcomes. Effective, rapid disbursement will depend on the capacity of these programmes to mobilise expertise, infrastructure, and sufficient human resources in a timely manner. Upfront support to national tuberculosis programmes to build the mechanisms to absorb new funding, and fully participate in resource allocation and management systems and processes within the health sector, will be crucial to ensure additional resources are 
appropriately used. The commitment of many highburden countries over the past two decades is commendable, and many have the space and willingness to do more, but achieving real increases in expenditures will require concerted attention by all those working to end tuberculosis to absorb additional resources effectively.

\section{Donor financing for tuberculosis}

The potential for increased domestic health spending and economic growth, along with the recent rise of populism and protectionism, ${ }^{364}$ will inevitably shape external financing for tuberculosis programmes over the coming decade. Nearly all high-burden countries can substantially increase domestic resources allocated to this disease. Although many low-income countries still require donor financing for tuberculosis, new opportunities exist to rethink how and where donor financing is allocated such that its effect is maximal. In this section, we discuss the role of donor financing to catalyse domestic efforts and invest in global public goods, especially in those countries transitioning out of donor finance eligibility. In addition, we highlight the potential benefits to donor partners of investing in tuberculosis, economically and in terms of addressing the negative cross-border externalities that tuberculosis, especially drug-resistant tuberculosis, poses. Finally, we underscore the importance of sustained financing for the poorest countries and advocate for continued investment to end the epidemic in those countries.

\section{Who is investing in tuberculosis programmes?}

According to the OECD's Creditor Reporting System, international donors provided US\$871 million for tuberculosis prevention, diagnosis, and treatment in 2016 (the latest year for which data are available); $69 \%$ of this funding was expended by the Global Fund, of which the USA was the major contributor. ${ }^{5}$ In addition, the US disbursed US\$179 million channelled via its own agencies and other institutions. Between 2006 and 2016, approximately $46 \%$ of international donor expenditure for tuberculosis originated in the USA. ${ }^{5}$ The next largest contributors were France $(10 \%)$, the UK $(9 \%)$, and Germany (6\%). ${ }^{5}$ According to the Institute for Health Metrics and Evaluation, The Bill \& Melinda Gates Foundation was the largest non-state funder of tuberculosis activities, responsible for US\$204 million of disbursements in 2016, including US\$68 million allocated to the Global Fund, whereas other sources of private philanthropy spent US\$70 million, of which $14 \%$ was allocated to the Global Fund. ${ }^{365}$

Development assistance for health (DAH) for tuberculosis has increased from US\$30 million in 1990 to over US $\$ 1$ billion in 2016, underscoring the substantial increases in international financing that have occurred over that period, as well as the relative contribution of foundations, development banks, the Global Fund, and traditional bilateral funding. Nonetheless, the amount of funding for tuberculosis is still very far short of the annual US\$2.6 billion proposed in the Global Plan to End TB, outlined by the Stop TB Partnership. ${ }^{158}$

\section{How is donor finance being used?}

Analyses of donor financing for health have traditionally tracked flows by funding source, channel, recipient, and disease. For this Commission, a team at University of California San Francisco and Duke University did an analysis of DAH for tuberculosis broken-down into functions ${ }^{61}$ Global functions refer to transnational topics, including supporting global public goods such as research and development, managing cross-border disease spread, and fostering leadership and stewardship. The researchers analysed DAH for tuberculosis in the year 2015, using the OECD Creditor Reporting System, which provides detailed information on aid expenditure. ${ }^{366}$ They found that in 2015, US\$932 million in DAH was directed towards tuberculosis-related activities. One-half of DAH for tuberculosis was disbursed to lower-middle income countries, $22 \%$ to low-income countries, $4 \%$ to upper middle-income countries, $23 \%$ to bilateral unspecified activities, and a small portion $(0.4 \%)$ to regional efforts. Only about one-quarter (24\%) of DAH for tuberculosis was for global functions, supporting product development (17\%), population, policy, and implementation research (3\%), advocacy and priority setting (2\%), and other global public goods (figure 11). Around three-quarters (76\%) of DAH for tuberculosis supported country-specific functions, including tuberculosis programmes for care delivery $(52 \%)$ and health system strengthening (24\%). Almost all (96\%) of the health system strengthening support was tuberculosisspecific, with only $4 \%$ directed at system-wide, crosscutting health system strengthening. These allocations

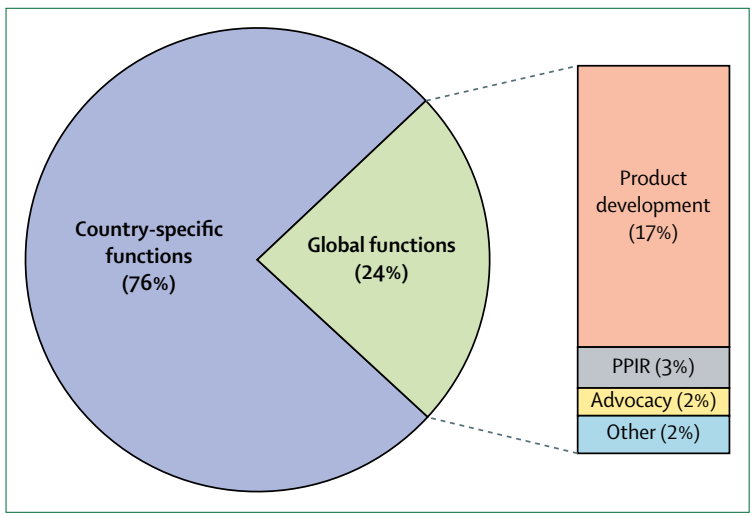

Figure 11: Breakdown of 2015 Development Assistance for Health for tuberculosis by global and country-specific functions Estimates are based on 2015 data from the Organisation for Economic Co-operation and Development, when US $\$ 932$ million of Development Assistance for Health was invested for tuberculosis control, through 907 tuberculosis projects. To estimate the proportion of funding directed at different functions, the researchers analysed the 141 largest individual projects (largest in terms of funding amount), representing $80 \%$ of all external funding for tuberculosis (US\$748 million). PPIR=population, policy, and implementation research. 
highlight that donor funds are being primarily targeted to support country-specific activities, especially those countries with the highest burden, rather than focused on global public goods.

\section{Policy implications}

To our knowledge, the analysis outlined in the previous section is the first to determine how much tuberculosisspecific DAH is devoted to support global functions versus country-specific functions. Notably, this analysis does not shed any light on trends in tuberculosis funding. It also does not distinguish between country-specific tuberculosis programme funding is disaggregated between drug-resistant tuberculosis versus drugsusceptible tuberculosis control efforts or provide granularity in terms of how DAH differs by disease burden or country income group. Nonetheless, the findings highlight the need to increase investment to support global tuberculosis functions, in addition to country-specific functions. Although this baseline analysis cannot prove that global functions are being neglected, prioritising funds to these global functions should be considered, especially as domestic resource allocation for tuberculosis increases. In particular, this Commission asserts that donor financing should increasingly be focused on global functions, as countries increase domestic investments in their tuberculosis control programmes. However, donor funds will still have a crucial role in supporting tuberculosis efforts in the poorest countries and in expanding services to vulnerable populations (appendix p 52).

Regarding global functions, financing should be provided for three main areas: supplying global public goods, market-shaping activities, and exercising leadership and advocacy. Greater investment in global public goods, in particular tuberculosis research and development of new drugs and technologies, is likely to bring important economic benefits and have a disproportionately beneficial effect on health outcomes in LMICs. ${ }^{113}$ New tools deriving from research and development are also likely to provide financial protection and be most beneficial to the members of society living in poorest conditions, as shown by extended costeffectiveness analyses. ${ }^{354}$ The investment in HIV research and development over the past two decades, leading to over 30 new drugs and numerous diagnostic and preventive technologies, provides compelling evidence for greater investment in tuberculosis research and development. ${ }^{293}$

The Global Drug Facility (GDF), part of the Stop TB Partnership, serves an important function in marketshaping activities, using donor financing to consolidate demand from different countries to negotiate lower prices for tuberculosis drugs, attract additional suppliers, and incentivise innovation, in particular for more expensive second-line agents and paediatric medicines. ${ }^{367-369}$ These kinds of activities will remain important as countries increasingly assume cofinancing responsibilities, transition out of donor eligibility, or both, as they might have difficulty negotiating lowest possible prices or accessing concessional prices for diagnostics. As countries move away from donor funding, the global market for tuberculosis medicines and diagnostics will probably become much more fragmented and the need for a global tuberculosis market steward, such as GDF, will become more important. In addition, the importance of GDF to facilitate uptake of new diagnostic and therapeutic tools will also be essential as investment in research and development yield greater successes in the coming years. ${ }^{370}$

An important, albeit often neglected, global function of aid is associated with investment in health advocacy and priority setting, which include, but are not limited to, donor financing to support civil society organisations (CSOs) as important catalysts for change. Although donor partners have increasingly committed to support community engagement efforts over the past decade, ${ }^{371} \mathrm{CSO}$ are still not recognised as legitimate partners at national levels, with their effect undermined by paucity of resources for community initiatives. ${ }^{372}$ Recognising that funding for HIV advocates and activists has been crucial to global HIV efforts, ${ }^{373,374}$ this Commission affirms the importance of increased funding for tuberculosis advocates as a public good, deserving investment commensurate with the part they plays in improving health outcomes.

Consideration should be given to increased investment in WHO's Global TB Programme, given its important role in facilitating uptake of new policies, strengthening surveillance systems, and providing technical assistance. A better-funded WHO would enable it to fulfil those functions more effectively. ${ }^{364}$ Independent regional initiatives, such as those established to tackle malaria, ${ }^{375}$ that can provide locally-relevant, agile, and responsive support to high-burden countries might also be worthy of donor investment.

Country-specific functions include tuberculosis programme activities, such as providing clinical and outreach services, as well as health systems that support tuberculosis, such as training providers and strengthening diagnostic facilities. Targeted investment is needed for countries graduating from DAH. 54\% of country-specific aid in our analysis is directed towards high-burden, middle-income countries, many of which will soon be ineligible for donor financing; based on their national GDP per capita, they are becoming too wealthy to qualify for DAH. Unfortunately, many of these countries are likely to have large pockets of poverty and avertable mortality from tuberculosis. In this Commission, we propose targeted investments directed to social insurance schemes that protect those at highest risk for tuberculosis. Furthermore, we argue that sustained funding in many of these countries, especially those with a significant drugresistant tuberculosis burden, is warranted given the 
global security implications of not ensuring tuberculosis control in these settings.

The high cost of treatment for drug-resistant tuberculosis, especially in middle-income countries, ${ }^{282}$ has been a substantial barrier to scale up treatment provision to date, and the cost will continue to increase over the coming years. ${ }^{67}$ Donor partners, especially the Global Fund, are already investing disproportionately in drug-resistant tuberculosis control activities. Nonetheless, given the substantial weight of data showing extensive cross-border spread of drug-resistant tuberculosis, ${ }^{376-386}$ this disease poses perplexing economic and health security issues for donor countries. It is important that sustained funding for drug-resistant tuberculosis control efforts, even in countries that will be soon no longer eligible for official development assistance, is available to mitigate the cross-border threat that drug-resistant tuberculosis poses. Aligning control efforts with the broader antimicrobial resistance agenda is also essential to maximise investment; unchecked tuberculosis will be the single biggest cause of antimicrobial resistancerelated deaths by $2050 .^{387}$

Prisoners, people living with tuberculosis and HIV coinfection, migrants, refugees, and indigenous populations are all highly vulnerable to tuberculosis, and experience substantial marginalisation, decreased access to quality services, and human rights violations. These communities will continue to benefit from donor support, for example, through support for social health insurance schemes that include tuberculosis services, ${ }^{354}$ even as domestic resources for health are increasing.

In addition to where DAH is spent, how it is spent is also crucial to guarantee the positive effect of donor support. Catalytic investments, such as those supported by the Global Fund, offer examples of how new models of financing can be useful. These models use matching funds to incentivise country allocation for priority areas or multicounty funding mechanisms that address specific priority areas (eg, development of innovative approaches to accelerate active case-finding, and scale up new tools or facilitating re-tooling initiatives, such as new drugs and diagnostics). ${ }^{388}$ Notwithstanding the need for improved data assessing the effect of these funding mechanisms, cofinancing solutions provide an important pathway to ensure greater country ownership while also ensuring sustained funding for tuberculosis activities even during the transition process.

Ongoing support is needed to help the poorest countries. By 2035, around two-dozen low-income countries are still likely to require direct country assistance. ${ }^{4}$ Donor financing for these countries needs to increase substantially to make up for funding shortfalls over the past few years. Despite a small increase in funding between 2016 and 2017 (US\$0.9 billion), donor-financing still fell very far short of the annual US $\$ 2.6$ billion in DAH that is needed for tuberculosis according to the Global Plan. ${ }^{5}$ The moral imperative of sustained donor investment in these countries should be highlighted as millions of individuals will potentially die from tuberculosis in these countries without external assistance. In addition, the scale of the effect of those avoidable deaths on the global economy is substantial. Investing in tuberculosis control will reap economic dividends that will benefit both donor and recipient nations. Underscoring the importance of investing in tuberculosis as an important tracer for progress towards $\mathrm{UHC}^{65}$ should also inform how and where donor funds are allocated. As global momentum builds towards achieving UHC, investment in tuberculosis as a disease of poverty is imperative to that progress.

\section{A new era of shared responsibility}

The UNHLM declaration and the stated commitment to shared responsibility highlighted how priorities and approaches to tuberculosis financing are evolving with a new era of increased country ownership and global cooperation. ${ }^{364,389}$ In addition, the architecture of donor financing for tuberculosis is changing as high-burden countries mobilise additional resources for tuberculosis control. Leveraging concessionary loans from development banks ${ }^{390}$ and innovative financing mechanisms (eg, social impact bonds, loan guarantees) ${ }^{160,364}$ should have an increased role. Such financing solutions have great potential, but they are no panacea. Strategies that can help increase domestic investment are crucial. Even in lowincome countries still reliant on donor support, the nature of donor-recipient financing must evolve. Partnership agreements between donors and recipients, as a tool to ensure ownership, accountability, and transparency, should be encouraged. Through this mechanism, donors could also help unlock domestic resources, by committing funds that pair global and national resources for shared priorities. ${ }^{391}$ New models of donor financing that focus on results, encourage innovation and strengthen government accountability to citizens rather than donors are also necessary. One promising example of a new financing strategy is the USAID's Global Accelerator to End TB, which was launched in September, 2018. The Accelerator will seek to link financial support with performance-based measures to maximise resources, whereas also leveraging additional resources from countries, private sector partners, and other local organisations. ${ }^{392}$ In addition to new funding mechanisms, new funding partners, such as multinational business and corporate philanthropists, should be encouraged to close tuberculosis funding gaps. The opportunity for legacy effects at national and global level, an often cited motivator of such funders, will increase as tuberculosis elimination efforts become tangible.

\section{Section 4: creating the enabling environment to end tuberculosis \\ Ending tuberculosis is important to achieve UHC}

As this Commission argues, progress towards ending tuberculosis should occur together with achieving UHC. 
UHC means all people have access to high-quality health services - at a minimum, health promotion and primary care-at no or little cost at the point of service. This Commission asserts that ending the tuberculosis pandemic must involve strong national tuberculosis programmes that can prioritise specific care and prevention functions within a progressive universalist pathway to UHC. This pathway is a publicly financed approach covering those core health-care services that directly benefit people living in poverty, who are disproportionally affected by tuberculosis. ${ }^{393}$ To this end, tuberculosis care and prevention functions have been addressed specifically and included within essential service packages. ${ }^{394,395}$ Social insurance models that prioritise diseases that disproportionately affect lowincome and other vulnerable populations will automatically incorporate tuberculosis. To realise the End TB targets, this Commission proposes to reach populations at highest risk for tuberculosis early in the roll-out of such schemes. In countries with high tuberculosis burdens, maintaining a separate tuberculosis budget and programme within a broader UHC framework typically will prove efficient. Even as the tuberculosis burden declines, ensuring that tuberculosis programmes maintain a very visible position within primary care budgets and Ministry of Health activities is advocated.

Several other system-wide frameworks are integral to a tuberculosis-inclusive UHC agenda. These frameworks include ensuring the uninterrupted availability of and access to appropriately regulated tuberculosis medications and diagnostic tests, strong information and performance systems, and new or merged risk financing pools. ${ }^{396}$ Regulation should address how medical products are subsidised, as well as the types of medical professionals authorised to prescribe or dispense tuberculosis medicines. High-burden countries will also need to establish an optimal mix of skilled health workers to deliver services and to design appropriate pay incentives for health professionals to support scaling up the tuberculosis response, as well as a broader UHC agenda. ${ }^{397}$ Robust information systems that are sensitive to tuberculosis indicators ${ }^{398}$ and infection control measures in health facilities are important. ${ }^{186}$ In addition, technical solutions applied to tuberculosis programmes, such as network optimisation and quality management, are essential to that UHC agenda and underscore how success in ending tuberculosis is tied to each country's success in ensuring high-quality health for all. ${ }^{399}$

\section{Social protection}

The adverse financial consequences of tuberculosis on households resulting from treatment costs and lost income during long periods of illness can be profound and long-lasting, as illustrated in panel 1 . To reduce the risk of impoverishment from tuberculosis requires policies that protect patients and their households against ruinous financial costs associated with tuberculosis. ${ }^{41}$ Especially in those settings where private sector care predominates, strategies that ensure financial protection and adequate quality of care must be adopted in both public and private sectors. This Commission argues that, as part of the UHC agenda, public finance should be extended to private providers for tuberculosis care, and that private finance in public facilities (user fees) should be minimised. Beyond public financing of treatment and case-finding, many patients with tuberculosis also might need economic and social support. These measures, particularly social support, can enhance treatment adherence and positively affect clinical outcomes. ${ }^{168}$

Social protection interventions $\mathrm{s}^{400}$ - policies and programmes designed to protect individuals from social and economic risks - are a promising approach to improve tuberculosis outcomes ${ }^{401,402}$ and achieve these larger policy goals. Examples include cash transfers and nutrition programmes offered as part of national policies. Such interventions can contribute to successful tuberculosis outcomes, either indirectly, by addressing social, biological, and structural determinants, or directly by enabling access to care..$^{78,403,404}$ These interventions can substantially affect tuberculosis trends by enhancing access to tuberculosis care and by mitigating the effect of tuberculosis-related catastrophic costs. ${ }^{405}$

\section{Sustaining top-level political support and leadership}

Strong national and local political leadership creates an environment conducive to sustained attention and funding. To end tuberculosis, governments of highburden countries will need to propose bold plans to end tuberculosis rather than be content with modest, incremental gains. Encouragingly, there is growing political recognition that countries need to act now to address the tuberculosis epidemic. Since its establishment in 2014, the Global TB Caucus, ${ }^{406}$ which supports 2300 parliamentarians in 130 countries, has become a driving force to mobilise political capital to address tuberculosis. Tuberculosis legislation in the Philippines ${ }^{407}$ and Peru, ${ }^{408}$ which mobilised national finances to drive improvements in tuberculosis care and prevention, highlights successes achieved because political leaders in these countries championed the cause. In South Africa, key political leaders from the Ministries of Health and Finance have been instrumental in formulating a tuberculosis investment case, to marshal additional resources to find new cases and treat more drug-resistant tuberculosis (figure 4). This investment case was developed with input from diverse stakeholders and as part of the planning process for the South African National Strategy Plan for HIV, tuberculosis and sexually transmitted infections. As figure 4 highlights, the investment case modelled the potential effect of increased domestic resources and was instrumental in ensuring political commitments from the South African government to double annual tuberculosis expenditure, with a goal of 
ensuring reduction in tuberculosis mortality rate by $87 \%$

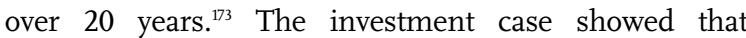
substantial scale up in tuberculosis and HIV programmes, necessary to achieve the End TB targets, would be costsaving after 2035. It provided the justification for the South African Treasury awarding South Africa's first-ever conditional grant for tuberculosis, which amounted to an increase in domestic funding for tuberculosis of 500 million South African Rand. Furthermore, it highlights that progress envisioned in the End TB strategy can be achieved only when each countries' leadership outlines a long-term strategy to combat tuberculosis within its borders.

Effective leadership within national tuberculosis programmes is also a crucial element of a successful tuberculosis response and evidence of high-level commitment to address tuberculosis. The size and capacity of these programmes central coordination team and the level of decentralisation and integration of specific services depend on many factors, including the country's size, governance, administrative structure, and tuberculosis epidemiology. However, chronic underinvestment in tuberculosis control efforts can undermine all aspects of tuberculosis programming, including the will and competency of key personnel, human resource planning, capacity strengthening, and service quality management. Empowering programme managers to take the necessary steps to institute effective strategies will require increased financing and recognition that national programme leaders must have an intersectoral, convening role with stakeholders of other government ministries, including finance, justice, labour, social welfare, housing, mining, and agriculture. Furthermore, a high priority must be placed on ensuring that these leaders have access to senior government leadership (Heads of Government and Ministers of Finance) who can authorise mobilisation of funds to realise the goals identified. To ensure the highcalibre of national tuberculosis programmes leadership needed to fulfil these expanded roles demands that these managers receive adequate pay, reasonable autonomy, and opportunities to maintain up-to-date technical knowledge.

\section{Maintaining multisectoral engagement}

In the SDG era, addressing tuberculosis must occur as part of a broader multisectoral framework that addresses key social determinants-especially poverty and overcrowding, ${ }^{409}$ malnutrition, ${ }^{9}$ smoking, ${ }^{410}$ and air pollution ${ }^{411}$ —clearly linked with tuberculosis incidence and mortality. Success will require collaboration among multiple ministries, agencies, and civil society. The health sector, particularly the national tuberculosis programme, can play a key role in identifying and communicating the potential health benefits of policies on food security, improved housing, poverty reduction, employment safeguards, and human rights protections for migrants, prisoners, and other marginalised groups. ${ }^{397}$ Numerous policy tools, including taxes and subsidies, laws and regulations, information and communication, and improvements in urban planning, should be employed to address these issues. Accountability to address these determinants at both a national and subnational level might be valuable, especially in addressing issues such as tobacco control and undernutrition.

Although not disavowing the additional value of a multisectoral agenda to address determinants of tuberculosis disease, this Commission recommends that improving access to diagnostic, treatment, and preventive services, especially for high-risk populations, should be the primary means of ending tuberculosis as a disease of global public health significance in all high-burden countries. Over the next generation, substantial progress can be made by ensuring that individuals with tuberculosis can access curative treatment, and that those at highest risk for tuberculosis disease can access preventive therapy, especially since so many do not have access. New investments are needed to make health systems more responsive and effective by providing greater access to rapid diagnosis and drug-susceptibility testing. ${ }^{286}$ Furthermore, new modalities for active casefinding, contact screening, and community-based care are urgently needed to close the substantial gap between unmet need and available resources. Continued improvements in tuberculosis control tools and delivery systems coupled with increased funding for health offer the most direct pathway to of ending the pandemic. ${ }^{114}$

\section{Strengthen civil society involvement in all aspects of the tuberculosis response}

A critical lesson learned from the HIV response is that engaging stakeholders from the civil, public, and private sectors requires national leadership to bring disparate actors together, overcome communication barriers, enable policies, and scale up access to effective medical tools. Civil society dramatically changed the global response to HIV, making it a top priority at all levels and driving unprecedented growth of donor support for lifesaving interventions. ${ }^{373,412}$

Until the past decade, few tuberculosis survivors or other people affected by the disease have served as public advocates, in part because of the disease's curable nature, the top-down orientation of tuberculosis control efforts, the persistent stigma of tuberculosis worldwide, and the scarcity of funding to support community involvement in tuberculosis programmes. ${ }^{413,414}$ However, a growing number of health-care workers and students who are tuberculosis survivors are using their dual perspectives and professional networks as platforms to call for rightsbased services and accelerated access to diagnostics, new treatment regimens, and vaccines..$^{413}$ National and transnational tuberculosis activism is emerging as a vital force advocating for services in hard-to-reach populations, mobilising communities and strengthening community systems. Tuberculosis survivors can play an essential role in creating incentives for political leaders to make 
difficult and risky decisions by generating public support for those decisions and holding leaders and service providers accountable for how resources, commitments, and services are delivered (examples in the appendix pp 57-59).

In the post-UNHLM era, the continued input of tuberculosis-affected civil actors is essential to ensure the accountability of politicians and programme planners. Recognising their contribution as a global public good, governments and international organisations must create conditions for civil society actors to have an expanded role in the fight against tuberculosis, supporting their contribution through direct investments and assembly to raise inconvenient truths. This contribution should include involving these advocates in national tuberculosis strategic planning processes, national tuberculosis research agenda setting activities, and national and regional accountability mechanisms.

\section{Strategies to reduce stigma and ensure a human rights-based approach to tuberculosis}

An important lesson from the HIV pandemic is that only by committing to universal human rights for everyone can the highest available standard of physical and mental health care be fulfilled. ${ }^{373}$ Upholding and defending the human rights of people with, or at most risk of, tuberculosis can decrease rates of infection and death. Practical solutions are needed to expedite changes in the laws, policies, and public attitudes that violate human rights of vulnerable populations who might be at particular risk of developing tuberculosis disease, including people living with HIV, prisoners, refugees and migrants, miners, and health-care workers. Furthermore, human rights must be an integral part of the design, implementation, and evaluation of an integrated and multisectoral response to tuberculosis. ${ }^{164}$ A human rights approach to tuberculosis research is required to ensure that legislative and policy frameworks exist to enable the widespread application of encouraging new scientific discoveries, provide accountability for research and development investments, ${ }^{415}$ and remove barriers that preclude new tuberculosis research technologies being broadly available for public benefit. ${ }^{416}$

In addition to addressing legal frameworks that undermine tuberculosis control efforts, action must be taken to address stigma, which is pervasive throughout health-care systems. Burdensome legal and social practices that systematically infantilise, impoverish, and expose people with or at risk of tuberculosis must be removed to end stigma. ${ }^{417,418}$ Public awareness campaigns that dispel unjustified fears and promote positive messages about tuberculosis, drawing on patient testimonials, can also help reduce stigmatising attitudes. ${ }^{419-421}$ Furthermore, campaigns that highlight the unfairness of obstacles faced by people with tuberculosis can evoke public support for greater investment in the welfare of stigmatised groups. ${ }^{422}$ Social protection interventions, such as conditional cash transfer programmes, can build resiliency to stigma, ${ }^{88,423-425}$ especially among patients whose self-identity and social capital are linked to their ability to sustain their families and themselves. ${ }^{426}$ It might also be useful to learn from and model successful campaigns from HIV, in which community engagement, advocacy, and political buy-in have aligned to ensure that policy making and programme planning mitigate stigma.

\section{WHO- a crucial role for a new era}

With greater emphasis on sustainable domestic resources and the centrality of national health systems, the SDG era also offers an opportunity to improve the definition of WHO's role in ending the tuberculosis epidemic. This Commission has identified several priorities for which WHO can be a leading catalyst for change. First, technical assistance to countries and strategic leadership might not be unique to WHO, but it must ensure that essential technical assistance is available to member states. ${ }^{364}$ Second, the WHO Global TB Programme must catalyse a rethinking of tuberculosis surveillance systems and the use of data platforms. In particular, WHO has a crucial role to play in modernising and expanding health information systems relevant to tuberculosis. Incorporating routine reporting of social protection indices and non-health SDGs into global tuberculosis reports is one key responsibility WHO has already embraced. ${ }^{398}$ However, by advocating for the improved use of subnational, realtime data and dashboard technologies, including performance data, WHO can encourage countries to use these systems to improve the quality and efficiency of their tuberculosis programmes, enable greater accountability, and facilitate more responsive and targeted technical assistance.

WHO's Director-General has repeatedly asserted the importance of UHC to his tenure, ${ }^{427}$ committing to "making universal health coverage happen in our lifetime". ${ }^{228}$ Accordingly, WHO must continue to support robust tuberculosis programmes as a central component of UHC. To end tuberculosis, both a focused commitment to tuberculosis activities and a progressive, inclusive vision of health care are essential. Thus, WHO must work to support countries to track indicators of tuberculosis coverage and social protection as important tracers of progress towards UHC.

\section{Establishing local, national, and global accountability}

Turning written commitments into substantive actions requires an accountability framework that tracks all elements of the tuberculosis response occurring at local, national, and global levels. This framework must measure progress towards ending tuberculosis worldwide and include timely reviews of results through government and civil society accountability mechanisms, both national and global. It must also incorporate a means for taking appropriate corrective actions. ${ }^{106}$ 
At a national level, this Commission proposes a framework to ensure that accountability extends beyond national tuberculosis programmes and reports directly to heads of state. Tuberculosis accountability should, as an exception, be reported to heads of government because of the health security risk that tuberculosis poses, and its adverse effect on national economies and health systems. Consistent with national strategic plans, such a framework should include specific targets for reducing mortality and detecting more cases, screening populations at high risk and scaling up access to preventive therapy, and addressing inequities in tuberculosis risk across populations. As highlighted in this Commission report, country-specific targets deriving from the global targets agreed upon at the UNHLM have been developed and provide benchmarks that all countries should achieve between 2018 and 2022. ${ }^{429}$ In addition, the framework also needs to ensure that financial resources are matched to achieve these targets. Furthermore, it should engage ministers across government to ensure multisectoral accountability on issues such as tobacco taxation and the regulation of air pollution, as well as progress towards addressing relevant SDGs. National tuberculosis commissions or cabinets that can either monitor progress across sectors, or ensure implementation of tuberculosis specific national strategic plans, or both, might be appropriate in high-burden countries. Enabling subnational accountability, using regional data to highlight gaps in services and opportunities for allocative efficiency, is also likely to be effective. Linking accountability mechanisms to financial resources that are allocated separately from health budgets can enable responsive, targeted responses. Such approaches have proven effective in addressing the HIV epidemic in several countries; ${ }^{373}$ given the health security risks and adverse economic impact of tuberculosis, similar approaches are justified to address the tuberculosis epidemic in many high-burden countries.

Separate mechanisms must also include accountability for nation states at a global level. We propose that heads of government should be accountable for their countries progress at the UN General Assembly on a biannual basis. This Commission asserts that accountability at the level of of the UN offers the best chance of driving global political action, and must be considered. Furthermore, the Commission recommends that mechanisms (eg, report cards or independent review processes) be established to hold countries accountable for their commitments and determine where additional assistance

\begin{tabular}{|c|c|c|c|c|c|c|c|c|c|c|c|c|c|}
\hline \multirow{2}{*}{\multicolumn{2}{|c|}{$\begin{array}{l}\text { Political will } \\
\text { High-level statement } \dagger\end{array}$}} & \multicolumn{4}{|c|}{$\begin{array}{l}\text { Progress on diagnosis and treatment } \\
\text { UN targets from } 2018-22^{*} \text { (thousand) }\end{array}$} & \multicolumn{4}{|c|}{ Tracers on equity and UHC } & \multicolumn{4}{|c|}{ Progress on relevant multisectoral and SDG targets } \\
\hline & & \multirow{2}{*}{$\begin{array}{l}\text { Drug- } \\
\text { susceptible } \\
\text { tuberculosis }\end{array}$} & \multirow{2}{*}{$\begin{array}{l}\text { Multi- } \\
\text { drug- } \\
\text { resistant } \\
\text { tuber- } \\
\text { culosis }\end{array}$} & \multirow{2}{*}{$\begin{array}{l}\text { Children } \\
\text { with } \\
\text { tuber- } \\
\text { culosis }\end{array}$} & \multirow{2}{*}{$\begin{array}{l}\text { Preventive } \\
\text { treatment } \\
\text { for tuber- } \\
\text { culosis }\end{array}$} & \multirow{2}{*}{$\begin{array}{l}\text { National } \\
\text { public- } \\
\text { sector } \\
\text { financing } \neq\end{array}$} & \multirow{2}{*}{$\begin{array}{l}\text { SDG } 1 \cdot 3 \\
\text { Social } \\
\text { protection } \\
\text { system } §\end{array}$} & \multirow{2}{*}{$\begin{array}{l}\text { SDG 3.8.2 } \\
\text { Catastrophic } \\
\text { health } \\
\text { expenditureq }\end{array}$} & \multirow{2}{*}{$\begin{array}{l}\text { SDG 3.8.1 } \\
\text { UHC } \\
\text { service } \\
\text { coverage } \\
\text { scorell }\end{array}$} & \multirow{2}{*}{$\begin{array}{l}\text { SDG 2.1 } \\
\text { Prevalence } \\
\text { of } \\
\text { under- } \\
\text { nourishment }\end{array}$} & \multirow{2}{*}{$\begin{array}{l}\text { SDG 3.3 } \\
\text { ART } \\
\text { access for } \\
\text { patients } \\
\text { with HIV** }\end{array}$} & \multirow[b]{2}{*}{$\begin{array}{l}\text { Tobacco } \\
\text { taxation }\end{array}$} & \multirow{2}{*}{$\begin{array}{l}\text { SDG 3.9 } \\
\text { Air } \\
\text { pollutiont† }\end{array}$} \\
\hline & & & & & & & & & & & & & \\
\hline & & $>90 \%$ & $>90 \%$ & $>90 \%$ & & $>15 \%$ & $>60 \%$ & $>13 \%$ & $\leq 45$ & $>5 \%$ & $>80 \%$ & $\begin{array}{c}\text { Yes } \\
(>25 \%)\end{array}$ & $<10 \mu \mathrm{g} / \mathrm{m}^{3}$ \\
\hline & & $60-90 \%$ & $60-90 \%$ & $60-90 \%$ & & $10-15 \%$ & $20-59 \%$ & $6-13 \%$ & $46-69$ & $5-20 \%$ & $60-79 \%$ & $\begin{array}{c}\text { Yes } \\
(>25 \%)\end{array}$ & $10-30 \mu \mathrm{g} / \mathrm{m}^{3}$ \\
\hline & & $<60 \%$ & $<60 \%$ & $<60 \%$ & & $<10 \%$ & $<20 \%$ & $<6 \%$ & $\geq 70$ & $>20 \%$ & $<60 \%$ & $\begin{array}{l}\text { No tax or } \\
\text { unknown }\end{array}$ & $>30 \mu \mathrm{g} / \mathrm{m}^{3}$ \\
\hline India & High & 11900 & 407 & 844 & 6997 & $5 \%$ & $19 \%$ & $17 \%$ & 56 & $15 \%$ & $56 \%$ & $43 \%$ & 65 \\
\hline Nigeria & High & 1180 & 57 & 118 & 2368 & $8 \%$ & $4 \%$ & $25 \%$ & 39 & $12 \%$ & $33 \%$ & $21 \%$ & 49 \\
\hline Indonesia & Moderate & 3445 & 58 & 416 & 1564 & $6 \%$ & & $4 \%$ & 49 & $8 \%$ & $14 \%$ & $57 \%$ & 16 \\
\hline South Africa & High & 1024 & 54 & 97 & 2677 & $14 \%$ & $48 \%$ & $1 \%$ & 67 & $6 \%$ & $61 \%$ & $52 \%$ & 24 \\
\hline Bangladesh & Low & 1535 & 17 & 110 & 969 & $6 \%$ & $28 \%$ & $0 \%$ & 46 & $15 \%$ & $19 \%$ & $77 \%$ & 58 \\
\hline Pakistan & Moderate & 2225 & 65 & 275 & 1669 & $5 \%$ & & $1 \%$ & 40 & $21 \%$ & $8 \%$ & $60 \%$ & 55 \\
\hline DR Congo & Low & 1117 & 18 & 135 & 1131 & $11 \%$ & $14 \%$ & $6 \%$ & 40 & $31 \%$ & $55 \%$ & $47 \%$ & 38 \\
\hline Tanzania & Low & 561 & 4 & 73 & 737 & $12 \%$ & & $10 \%$ & 39 & $32 \%$ & $66 \%$ & $34 \%$ & 26 \\
\hline Mozambique & Moderate & 637 & 21 & 87 & 1124 & $9 \%$ & $11 \%$ & $1 \%$ & 42 & $31 \%$ & $54 \%$ & $31 \%$ & 19 \\
\hline Kenya & High & 564 & 7 & 65 & 950 & $13 \%$ & $10 \%$ & $6 \%$ & 57 & $24 \%$ & $75 \%$ & $52 \%$ & 26 \\
\hline
\end{tabular}


is needed. This approach has been an effective political component of the global fight to end HIV, as it has maintained global recognition and financial investment to address this disease. Although the details of any national report card would need to be drafted and approved to ensure stakeholder consensus, commitments on accountability should include progress towards key End TB milestones and other relevant SDGs; adoption and implementation of WHO recommended policies; registration of and access to the newest and best medical tools; and tuberculosis financing. ${ }^{106}$ Figure 12 gives an example of a report card, highlighting the performance of ten high tuberculosis burden countries on several epidemiologic, programmatic, financial, and multisectoral indicators.

Finally, OECD donor countries, international multilateral funding agencies (such as the Global Fund and UNITAID), non-governmental funders (eg, Bill \& Melinda Gates Foundation), and the agencies of the UN (including WHO, UNICEF, and UNAIDS) have crucial roles in global efforts to end tuberculosis, for which they also must be held accountable. Leveraging the quality of official development assistance metrics already published by the Centre for Global Development, ${ }^{434}$ a report card that highlights strengths and weaknesses of major bilateral tuberculosis donors is shown in the appendix ( $p 53$ ). Its purpose is to illustrate metrics on which these donors can be evaluated. Donor accountability to address drugresistant tuberculosis and tuberculosis research and development must be a focus in these report cards, including the allocation of funds to address drugresistant tuberculosis-related activities, the investment in research and development, or both. Similar report cards for multilateral funders and major non-state actors are also necessary to ensure that these institutions also are held accountable for their efforts towards ending the pandemic, and to ensure that investments are synergistic with domestic investments. Enhanced accountability of these institutions, not just to their board members or citizenry, but to tuberculosis survivors and their advocates in recipient countries, represents a global public good. Although the indicators and governance for these proposed report cards will need to be drafted and agreed to by consensus, dimensions should include performance monitoring and assessment, efficiency and effectiveness, sustainability, transparency, and responsiveness to corrective feedback.

\section{The Lancet Tuberculosis Observatory}

To spur political action and monitor progress towards ending tuberculosis after the UNHLM on Tuberculosis, The Lancet Commission and experts participating in this Commission will launch The Lancet Tuberculosis Observatory. The idea for this Observatory was first proposed in 2010 to promote urgent global action to control the tuberculosis pandemic. ${ }^{17}$ It is needed now more than ever. The Observatory will be composed of global experts, tuberculosis survivors and their advocates, and multisectoral stakeholders from high-burden countries and will meet annually between now and 2022 to critically evaluate progress towards targets made at the UNHLM. Leveraging the tuberculosis report card, it also will monitor domestic and global financing for efforts to End TB and identify corrective actions and investments necessary to achieve targets. By providing an independent perspective on the activities of key global stakeholders, including WHO, the Stop Tuberculosis Partnership, and the Global Fund, The Lancet Tuberculosis Observatory can also help optimise alignment of these different bodies towards ending the pandemic.

\section{Conclusions}

We can build a tuberculosis-free world. Many countrieseven many LMICS-have shown that it is achievable, despite the limitations of existing tools. The prospect of a tuberculosis-free world is not just a distant aspiration. It is a realistic objective that can be achieved with the right commitment of leadership and resources. It will be a difficult task, with potential setbacks including the challenge of drug-resistance, funding obstacles, and uncertainties about the correct prioritisation of tools and implementation approaches.

In the short term, however, the UNHLM targets provide concrete, achievable, reasonable goals that all countries can strive towards. Early and aggressive investment towards meeting these targets will have a great impact on the trajectory of the pandemic and save money and resources in the long term. We hope that the recommendations and supporting evidence provided in this Commission report give countries a roadmap to achieve these goals and end their tuberculosis epidemics. With targeted, proven strategies, smart investments based on sound science, accelerated research and development, and a shared responsibility, we can defeat tuberculosis within a generation.

\section{Contributors}

The first draft of this report was written by a core writing team led by MJAR, which also included DTJ, NA, and EPG. All Commissioners contributed fully to the overall report structure and concepts, the writing and editing of subsequent drafts, and the conclusions. The report was prepared under the general direction of EPG, and co-chair DTJ Introductory section was drafted with input of MJAR, MP, TP, DC, LM, MCR, and DJT. Modelling analyses highlighted in sections 1 and 2 were done by NA, JFV, and GBG. Section 1 was written by a writing team that included inputs from MJAR, CLH, CB, DC, GS, REC, BDA, AC, MP, $\mathrm{HC}, \mathrm{JF}$, and PCH. Private sector analysis were done by GS, PD, and DPC Section 2 had crucial inputs from AF, BRB, CB, MH, RWE, HC, NB, and GY. Economic analyses in this section were done by NA, VF, and DTJ. The section 3 writing team included DTJ, AV, GY, and MJAR, with inputs from SM, MD, and IK. Domestic resource analysis in this section was undertaken by $\mathrm{AV}, \mathrm{MR}$, and SF. Donor financing by function analysis was done by SF, NB, and GY. Section 4 was written by MJAR and DJT, with inputs from MR and PS. Additional analysis on financial cost of tuberculosis disease in India was done by JDG-F, LP, and JRA. Inputs on paediatric content was provided by JAS, SMG, and SS. Data gathering was done also by a supporting research team listed in the Acknowledgments. In addition to the participation and inputs of all Commission members, several additional contributory authors prepared background papers and 
analyses to support the main theses of the report. The views expressed herein are those of the authors themselves and do not necessarily represent the views of the institutions by whom they are employed.

\section{Declaration of interests}

$\mathrm{RC}$ reports personal fees from Merck and Otsuka; and grants from US National Institutes of Health (NIH), Centers for Disease Control and Prevention, and Gilead Foundation, outside the submitted work. HC reports non-financial support from US Agency for International Development (USAID), during this study; and grants from Wellcome Trust, South Africa National Research Foundation, and the UK Medical Research Council, outside the submitted work. EPG reports grants from USAID, during this study. TBH reports grants from Gilead Sciences, during this study; grants from BMGF, World Bank, UNAIDS, Rush Foundation, and Wellcome Trust, outside the submitted work; and personal fees from Bill \& Melinda Gates Foundation, New York University, WHO, and The Global Fund to Fight Aids, Tuberculosis and Malaria, outside the submitted work. CLH reports grants and personal fees from Bill \& Melinda Gates Foundation, during this study. TAG has received financial support for its tuberculosis and HIV advocacy project from the Bill \& Melinda Gates Foundation, WHO, Stop TB Partnership, Unitaid (via Aurum and Elizabeth Glaser Pediatric AIDS Foundation), the Veterans Administration, Harvard University, and the Stony Wold-Herbert Foundation; is a member of the US Adult AIDS Clinical Trials Group Tuberculosis Transformative Study Group (TB TSG), of the protocol team of the forthcoming PHOENIx MDR-TB (Protecting Households On Exposure to Newly Diagnosed Index Multidrug-Resistant Tuberculosis Patients) trial, of the Scientific Advisory Board of the John Hopkins University Center for AIDS Research (also provides travel support), and of the PEPFAR Scientific Advisory Board of the Office of the Global AIDS Coordinator (also provides travel support); and reports further travel support by the National Institute of Allergy and Infectious Diseases and the National Institute of Child Health and Human Development, University of California San Francisco and San Francisco General Hospital, and Johns Hopkins University Center for AIDS Research. NH is a member of the British Parliament and co-chair of the Global TB Caucus. MP is a member of WHO's Strategic and Technical Advisory Group for Tuberculosis Committee, Access Advisory Committee of TB Alliance, and Scientific Advisory Committee of FIND. PMS reports a patent (US patent number 7700118 on April 20, 2010 licensed). AV reports personal fees from Johnson and Johnson, outside the submitted work. GY reports a grant from the Bill \& Melinda Gates Foundation, during this study. SF reports a grant from the Bill \& Melinda Gates Foundation, during this study. GBG reports personal fees from Bill \& Melinda Gates Foundation, outside the submitted work. MO reports support from Bill \& Melinda Gates Foundation, during this study and outside the submitted work; and is a senior associate of Linksbridge, which provides consulting services to the Bill \& Melinda Gates Foundation. GS reports personal fees from Bill \& Melinda Gates Foundation, during the conduct of the study. The remaining authors declare no competing interests

\section{Acknowledgments}

This Commission was supported financially by the US Agency for International Development (USAID). The funding covered travel, accommodation, and meals for the three face-to-face meetings of the Commission; research assistance time and the Commission secretariat, based at University of California San Francisco, San Francisco, CA, USA; and additional modelling analysis done by a team from Imperial College, London, UK (also supported by the Stop TB Partnership). Both Stop TB Partnership and University Research Company provided invaluable administrative support to the Commission. We are grateful for the input of additional consultants, experts, and stakeholders who contributed with invaluable insights and perspectives through a variety of consultations. We acknowledge the contributions of Ramnath Subbaraman for his detailed exposition of care cascade analyses, Mustapha Gidado for his honest and provocative insights into the challenges of running a National TB program, Matthew Oliver who provided an invaluable perspective on the political economy of tuberculosis, Cecily Miller's critical analysis of the state of active case finding, and Anthony Harries whose vision and insights had a formative role in shaping the vision for the Commission. Claudia Denkinger, at FIND, had invaluable roles in assisting with the research and development section. We are grateful for the input of experts from Stop TB Partnership including Sahu Suvanand and Brenda Waning, whose contributions were particularly useful in the writing of section 3 of the Commission. We thank the faculty and staff at the Institute for Global Health Sciences at University of California San Francisco who prepared analytic inputs and technical briefs in preparation for Commission meetings. We highlight Jane Coyne, David McKey, Anne Wolfe, Kerstin Svendsen, Geoff Daily, Colin Cooper, Pal Shah, Nazila Dabistani, and Glenna Roberts whose hard work and attention to detail were indispensable. We are also grateful for Jaime Sepulveda's leadership and support for the endeavor. The Commission also thanks the anonymous referees for their outstanding peer review and Salman Keshavjee for providing rigorous, thoughtful and provocative in-person critique of the report at a critical juncture. Finally, we are grateful for the insights and comments of the tuberculosis survivors who shared their perspectives, Endy Fekadu, Nandita Venkatesan, Paulina Siniatkina, and Jo Chandler.

\section{References}

1 Nakajima H. Tuberculosis: a global emergency. Geneva, Switzerland: World Health Organization, 1993.

2 Raviglione MC, Uplekar MW. WHO's new Stop TB Strategy. Lancet 2006; 367: 952-55.

3 Uplekar M, Raviglione M. WHO's End TB Strategy: From stopping to ending the global TB epidemic. Indian J Tuberc 2015; 62: 196-99.

4 Uplekar M, Weil D, Lonnroth K, et al. WHO's new end TB strategy. Lancet 2015; 385: 1799-801.

5 WHO. Global tuberculosis report 2018. Geneva: World Heath Organization, 2018.

6 Dodd PJ, Yuen CM, Sismanidis C, Seddon JA, Jenkins HE. The global burden of tuberculosis mortality in children: a mathematical modelling study. Lancet Glob Health 2017; 5: e898-906.

7 WHO. Disease burden and mortality estimates. 2018. https://www. who.int/healthinfo/global_burden_disease/estimates/en/ (accessed Jan 31, 2019).

8 GBD 2017 Causes of Death Collaborators. Global, regional, and national age-sex-specific mortality for 282 causes of death in 195 countries and territories, 1980-2017: a systematic analysis for the Global Burden of Disease Study 2017. Lancet 2018; 392: 1736-88.

9 Ortblad KF, Salomon JA, Bärnighausen T, Atun R. Stopping tuberculosis: a biosocial model for sustainable development. Lancet 2015; 386: 2354-62.

10 Oxlade O, Piatek A, Vincent C, Menzies D. Modeling the impact of tuberculosis interventions on epidemiologic outcomes and health system costs. BMC Public Health 2015; 15: 141.

11 Arinaminpathy N, Dowdy D. Understanding the incremental value of novel diagnostic tests for tuberculosis. Nature 2015; 528: S60-67.

12 Chikovore J, Hart G, Kumwenda M, Chipungu GA, Corbett L. 'For a mere cough, men must just chew Conjex, gain strength, and continue working': the provider construction and tuberculosis care-seeking implications in Blantyre, Malawi. Glob Health Action 2015; 8: 26292.

13 Horton KC, MacPherson P, Houben RM, White RG, Corbett EL. Sex differences in tuberculosis burden and notifications in low- and middle-income countries: a systematic review and meta-analysis. PLoS Med 2016; 13: e1002119.

14 Watkins DA, Yamey G, Schaferhoff M, et al. Alma-Ata at 40 years: reflections from the Lancet Commission on Investing in Health. Lancet 2018; 392: 1434-60.

15 Lancet. Declaration of Alma-Ata. Lancet 1978; 2: 1040-41.

16 Pai M, Furin J. Tuberculosis innovations mean little if they cannot save lives. eLife 2017; 6: 6.

17 Onyebujoh P, Rodriguez W, Mwaba P. Priorities in tuberculosis research. Lancet 2006; 367: 940-42.

18 Marais BJ, Raviglione MC, Donald PR, et al. Scale-up of services and research priorities for diagnosis, management, and control of tuberculosis: a call to action. Lancet 2010; 375: 2179-91.

19 Keshavjee S, Dowdy D, Swaminathan S. Stopping the body count: a comprehensive approach to move towards zero tuberculosis deaths. Lancet 2015; 386: e46-47.

20 Hanson C, Osberg M, Brown J, Durham G, Chin DP. Finding the missing patients with tuberculosis: lessons learned from patient-pathway analyses in 5 countries. J Infect Dis 2017; 216 (suppl 7): S686-95. 
21 Chin DP, Hanson CL. Finding the Missing tuberculosis patients. J Infect Dis 2017; 216 (suppl 7): S675-78.

22 Masini E, Hanson C, Ogoro J, et al. Using patient-pathway analysis to inform a differentiated program response to tuberculosis: the case of Kenya. J Infect Dis 2017; 216 (suppl 7): S714-23.

23 Surya A, Setyaningsih B, Suryani Nasution H, et al. Quality tuberculosis care in Indonesia: using patient pathway analysis to optimize public-private collaboration. J Infect Dis 2017; 216 (suppl 7): S724-32.

24 Shete PB, Haguma P, Miller CR, et al. Pathways and costs of care for patients with tuberculosis symptoms in rural Uganda. Int J Tuberc Lung Dis 2015; 19: 912-17.

25 Kemp JR, Mann G, Simwaka BN, Salaniponi FM, Squire SB. Can Malawi's poor afford free tuberculosis services? Patient and household costs associated with a tuberculosis diagnosis in Lilongwe. Bull World Health Organ 2007; 85: 580-85.

26 Sreeramareddy CT, Panduru KV, Menten J, Van den Ende J. Time delays in diagnosis of pulmonary tuberculosis: a systematic review of literature. BMC Infect Dis 2009; 9: 91.

27 Kruk ME, Gage AD, Arsenault C, et al. High-quality health systems in the Sustainable Development Goals era: time for a revolution. Lancet Glob Health 2018; 6: e1196-252.

28 Medecins Sans Frontieres. Out of Step 2017: TB policies in 29 countries, 2017

29 Stop TB Partnership. Racing to the end TB finish line: tuberculosis financing in 2014. Geneva: World Health Organization, 2014. http://www.stoptb.org/assets/documents/news/Factsheet_05.pdf (accessed Feb 22, 2019).

30 Huddart S, MacLean E, Pai M. Location, location, location: tuberculosis services in highest burden countries. Lancet Glob Health 2016; 4: e907-08.

31 Sreeramareddy CT, Qin ZZ, Satyanarayana S, Subbaraman R, Pai M. Delays in diagnosis and treatment of pulmonary tuberculosis in India: a systematic review. Int J Tuberc Lung Dis 2014; 18: 255-66.

32 Cazabon D, Suresh A, Oghor C, et al. Implementation of Xpert MTB/RIF in 22 high tuberculosis burden countries: are we making progress? Eur Respir J 2017; 50: pii: 1700918.

33 Das J, Kwan A, Daniels B, et al. Use of standardised patients to assess quality of tuberculosis care: a pilot, cross-sectional study. Lancet Infect Dis 2015; 15: 1305-13.

34 Daniels B, Dolinger A, Bedoya G, et al. Use of standardised patients to assess quality of healthcare in Nairobi, Kenya: a pilot, cross-sectional study with international comparisons. BMJ Glob Health 2017; 2: e000333.

35 Sylvia S, Xue H, Zhou C, et al. Tuberculosis detection and the challenges of integrated care in rural China: a cross-sectional standardized patient study. PLoS Med 2017; 14: e1002405.

36 Cazabon D, Alsdurf H, Satyanarayana S, et al. Quality of tuberculosis care in high burden countries: the urgent need to address gaps in the care cascade. Int J Infect Dis 2017; 56: 111-16.

37 Subbaraman R, Nathavitharana RR, Satyanarayana S, et al. The tuberculosis cascade of care in India's public sector: a systematic review and meta-analysis. PLoS Med 2016; 13: e1002149.

38 Naidoo P, Theron G, Rangaka MX, et al. The South African tuberculosis care cascade: estimated losses and methodological challenges. J Infect Dis 2017; 216 (suppl 7): S702-13.

39 Alsdurf H, Hill PC, Matteelli A, Getahun H, Menzies D. The cascade of care in diagnosis and treatment of latent tuberculosis infection: a systematic review and meta-analysis. Lancet Infect Dis 2016; 16: 1269-78.

40 MacPherson P, Houben RM, Glynn JR, Corbett EL, Kranzer K. Pre-treatment loss to follow-up in tuberculosis patients in low- and lower-middle-income countries and high-burden countries: a systematic review and meta-analysis. Bull World Health Organ 2014 92: $126-38$.

41 Tanimura T, Jaramillo E, Weil D, Raviglione M, Lönnroth K. Financial burden for tuberculosis patients in low- and middle-income countries: a systematic review. Eur Respir J 2014 43: 1763-75.

42 Satyanarayana S, Kwan A, Daniels B, et al. Use of standardised patients to assess antibiotic dispensing for tuberculosis by pharmacies in urban India: a cross-sectional study. Lancet Infect Dis 2016; 16: 1261-68.
43 Padayatchi N, Daftary A, Naidu N, Naidoo K, Pai M. Tuberculosis: treatment failure, or failure to treat? Lessons from India and South Africa. BMJ Glob Health 2019; 4: e001097.

44 Subbaraman R, Nathavitharana RR, Mayer KH, et al. Constructing care cascades for active tuberculosis: a strategy for program monitoring and identifying gaps in quality of care. PLoS Med 2019; 16: e1002754.

45 Mwangwa F, Chamie G, Kwarisiima D, et al. Gaps in the child tuberculosis care cascade in 32 rural communities in Uganda and Kenya. J Clin Tuberc Other Mycobact Dis 2017; 9: 24-29.

46 Kwan A, Daniels B, Saria V, et al. Variations in the quality of tuberculosis care in urban India: a cross-sectional, standardized patient study in two cities. PLoS Med 2018; 15: e1002653.

47 Arinaminpathy N, Batra D, Khaparde S, et al. The number of privately treated tuberculosis cases in India: an estimation from drug sales data. Lancet Infect Dis 2016; 16: 1255-60.

48 Houben RMGJ, Menzies NA, Sumner T, et al. Feasibility of achieving the 2025 WHO global tuberculosis targets in South Africa, China, and India: a combined analysis of 11 mathematical models. Lancet Glob Health 2016; 4: e806-15.

49 Dhanaraj B, Papanna MK, Adinarayanan S, et al. Prevalence and risk factors for adult pulmonary tuberculosis in a metropolitan city of South India. PLoS One 2015; 10: e0124260.

50 Chadha VK. Tuberculosis epidemiology in India: a review. Int J Tuberc Lung Dis 2005; 9: 1072-82.

51 MacIntyre CR, Kendig N, Kummer L, Birago S, Graham NM. Impact of tuberculosis control measures and crowding on the incidence of tuberculous infection in Maryland prisons. Clin Infect Dis 1997; 24: 1060-67.

52 Chee CB, Teleman MD, Boudville IC, Wang YT. Contact screening and latent TB infection treatment in Singapore correctional facilities. Int J Tuberc Lung Dis 2005; 9: 1248-52.

53 Hanifa Y, Grant AD, Lewis J, Corbett EL, Fielding K, Churchyard G. Prevalence of latent tuberculosis infection among gold miners in South Africa. Int J Tuberc Lung Dis 2009; 13: 39-46.

54 Isler MA, Rivest P, Mason J, Brassard P. Screening employees of services for homeless individuals in Montréal for tuberculosis infection. J Infect Public Health 2013; 6: 209-15.

55 van Rie A, McCarthy K, Scott L, Dow A, Venter WD, Stevens WS. Prevalence, risk factors and risk perception of tuberculosis infection among medical students and healthcare workers in Johannesburg, South Africa. S Afr Med J 2013; 103: 853-57.

56 Kranzer K, Afnan-Holmes H, Tomlin K, et al. The benefits to communities and individuals of screening for active tuberculosis disease: a systematic review. Int J Tuberc Lung Dis 2013; 17: 432-46.

57 Houben RM, Dodd PJ. The global burden of latent tuberculosis infection: a re-estimation using mathematical modelling. PLoS Med 2016; 13: e1002152.

58 Lincoln EM. The effect of antimicrobial therapy on the prognosis of primary tuberculosis in children. Am Rev Tuberc 1954; 69: 682-89.

59 Comstock GW, Baum C, Snider DE Jr. Isoniazid prophylaxis among Alaskan Eskimos: a final report of the bethel isoniazid studies. Am Rev Respir Dis 1979; 119: 827-30.

60 Ferebee SH. Controlled chemoprophylaxis trials in tuberculosis. A general review. Bibl Tuberc 1970; 26: 28-106.

61 Rangaka MX, Wilkinson RJ, Boulle A, et al. Isoniazid plus antiretroviral therapy to prevent tuberculosis: a randomised double-blind, placebo-controlled trial. Lancet 2014; 384: 682-90.

62 Askling J, Fored CM, Brandt L, et al. Risk and case characteristics of tuberculosis in rheumatoid arthritis associated with tumor necrosis factor antagonists in Sweden. Arthritis Rheum 2005; 52: 1986-92.

63 Getahun H, Chaisson RE, Raviglione M. Latent Mycobacterium tuberculosis infection. N Engl J Med 2015; 373: 1179-80.

64 Mills HL, Cohen T, Colijn C. Modelling the performance of isoniazid preventive therapy for reducing tuberculosis in HIV endemic settings: the effects of network structure. J R Soc Interface 2011; 8: 1510-20.

65 Dowdy DW, Golub JE, Saraceni V, et al. Impact of isoniazid preventive therapy for HIV-infected adults in Rio de Janeiro, Brazil: an epidemiological model. J Acquir Immune Defic Syndr 2014; 66: 552-58. 
66 Churchyard GJ, Chaisson RE, Maartens G, Getahun H. Tuberculosis preventive therapy: an underutilised strategy to reduce individual risk of TB and contribute to TB control. S Afr Med J 2014; 104: 339-43.

67 World Health Organization. Global Tuberculosis Report 2017. Geneva: World Health Organization, 2017.

68 Jenkins HE, Plesca V, Ciobanu A, et al. Assessing spatial heterogeneity of multidrug-resistant tuberculosis in a high-burden country. Eur Respir J 2013; 42: 1291-301.

69 Zelner JL, Murray MB, Becerra MC, et al. Identifying hotspots of multidrug-resistant tuberculosis transmission using spatial and molecular genetic data. J Infect Dis 2016; 213: 287-94.

70 Udwadia ZF, Amale RA, Ajbani KK, Rodrigues C. Totally drug-resistant tuberculosis in India. Clin Infect Dis 2012; 54: 579-81.

71 Zhao Y, Xu S, Wang L, et al. National survey of drug-resistant tuberculosis in China. N Engl J Med 2012; 366: 2161-70.

72 Shah NS, Auld SC, Brust JC, et al. Transmission of extensively drug-resistant tuberculosis in South Africa. N Engl J Med 2017; 376: 243-53.

73 Yang C, Luo T, Shen X, et al. Transmission of multidrug-resistant Mycobacterium tuberculosis in Shanghai, China: a retrospective observational study using whole-genome sequencing and epidemiological investigation. Lancet Infect Dis 2017; 17: 275-84.

74 Kendall EA, Fofana MO, Dowdy DW. Burden of transmitted multidrug resistance in epidemics of tuberculosis: a transmission modelling analysis. Lancet Respir Med 2015; 3: 963-72.

75 Dowdy DW, Golub JE, Chaisson RE, Saraceni V. Heterogeneity in tuberculosis transmission and the role of geographic hotspots in propagating epidemics. Proc Natl Acad Sci USA 2012; 109: 9557-62.

76 Lönnroth K, Glaziou P, Weil D, Floyd K, Uplekar M, Raviglione M. Beyond UHC: monitoring health and social protection coverage in the context of tuberculosis care and prevention. PLoS Med 2014; 11: e1001693.

77 Lönnroth K, Jaramillo E, Williams BG, Dye C, Raviglione M. Drivers of tuberculosis epidemics: the role of risk factors and social determinants. Soc Sci Med 2009; 68: 2240-46.

78 Hargreaves JR, Boccia D, Evans CA, Adato M, Petticrew M, Porter JD. The social determinants of tuberculosis: from evidence to action. Am J Public Health 2011; 101: 654-62.

79 Creswell J, Jaramillo E, Lönnroth K, Weil D, Raviglione M. Tuberculosis and poverty: what is being done. Int J Tuberc Lung Dis 2011; 15: 431-32.

80 Adeyi O, Darley S, Floyd K, Dye C, Klein E, Laxminarayan R. Economic benefit of tuberculosis control. Washington, DC: World Bank, 2007.

81 Ukwaja KN, Modebe O, Igwenyi C, Alobu I. The economic burden of tuberculosis care for patients and households in Africa: a systematic review. Int J Tuberc Lung Dis 2012; 16: 733-39.

82 Kapoor SK, Raman AV, Sachdeva KS, Satyanarayana S. How did the TB patients reach DOTS services in Delhi? A study of patient treatment seeking behavior. PLoS One 2012; 7: e42458.

83 Mauch V, Bonsu F, Gyapong M, et al. Free tuberculosis diagnosis and treatment are not enough: patient cost evidence from three continents. Int J Tuberc Lung Dis 2013; 17: 381-87.

84 Aspler A, Menzies D, Oxlade O, et al. Cost of tuberculosis diagnosis and treatment from the patient perspective in Lusaka, Zambia. Int J Tuberc Lung Dis 2008; 12: 928-35.

85 WHO. Global tuberculosis report 2017. Geneva: World Health Organization, 2017.

86 Boccia D, Hargreaves J, De Stavola BL, et al. The association between household socioeconomic position and prevalent tuberculosis in Zambia: a case-control study. PLoS One 2011; 6: e20824.

87 Richter LM, Lönnroth K, Desmond C, Jackson R, Jaramillo E, Weil D. Economic support to patients in HIV and TB grants in rounds 7 and 10 from the global fund to fight AIDS, tuberculosis and malaria. PLoS One 2014; 9: e86225.

88 Sripad A, Castedo J, Danford N, Zaha R, Freile C. Effects of Ecuador's national monetary incentive program on adherence to treatment for drug-resistant tuberculosis. Int J Tuberc Lung Dis 2014 18: $44-48$.

89 Chandra S, Sharma N, Joshi K, Aggarwal N, Kannan AT. Resurrecting social infrastructure as a determinant of urban tuberculosis control in Delhi, India. Health Res Policy Syst 2014; 12: 3.
90 Dye C, Lönnroth K, Jaramillo E, Williams BG, Raviglione M. Trends in tuberculosis incidence and their determinants in 134 countries. Bull World Health Organ 2009; 87: 683-91.

91 Álvarez JL, Kunst AE, Leinsalu M, et al. Educational inequalities in tuberculosis mortality in sixteen European populations. Int J Tuberc Lung Dis 2011; 15: 1461-67, i.

92 Rasanathan K, Sivasankara Kurup A, Jaramillo E, Lönnroth K The social determinants of health: key to global tuberculosis control. Int J Tuberc Lung Dis 2011; 15 (suppl 2): 30-36.

93 Lin HH, Ezzati M, Murray M. Tobacco smoke, indoor air pollution and tuberculosis: a systematic review and meta-analysis. PLoS Med 2007; 4: e20.

94 Graham SM, Sismanidis C, Menzies HJ, Marais BJ, Detjen AK Black RE. Importance of tuberculosis control to address child survival. Lancet 2014; 383: 1605-07.

95 WHO. Global tuberculosis report. Geneva: World Health Organization, 2017. https://www.who.int/tb/publications/global_ report/gtbr2017_main_text.pdf (accessed Feb 23, 2019).

96 Dodd PJ, Sismanidis C, Seddon JA. Global burden of drug-resistant tuberculosis in children: a mathematical modelling study. Lancet Infect Dis 2016; 16: 1193-201.

97 Jenkins HE, Tolman AW, Yuen CM, et al. Incidence of multidrugresistant tuberculosis disease in children: systematic review and global estimates. Lancet 2014; 383: 1572-79.

98 WHO. The End TB Strategy. Geneva: World Health Organization, 2015. http://www.who.int/tb/strategy/End_TB_Strategy.pdf?ua=1 (accessed Nov 15, 2016).

99 WHO. Roadmap for childhood tuberculosis. Geneva: World Health Organization. 2018. https://apps.who.int/iris/bitstream/hand le/10665/275422/9789241514798-eng.pdf?ua=1 (accessed Feb 23, 2019).

100 Graham SM. The management of infection with Mycobacterium tuberculosis in young children post-2015: an opportunity to close the policy-practice gap. Expert Rev Respir Med 2017; 11: 41-49.

101 Chisti MJ, Ahmed T, Pietroni MA, et al. Pulmonary tuberculosis in severely-malnourished or HIV-infected children with pneumonia: a review. J Health Pop Nutr 2013; 31: 308-13.

102 Pai M, Behr MA, Dowdy D, et al. Tuberculosis. Nat Rev Dis Primers 2016; 2: 16076

103 Dowdy DW, Azman AS, Kendall EA, Mathema B. Transforming the fight against tuberculosis: targeting catalysts of transmission. Clin Infect Dis 2014; 59: 1123-29.

104 Frieden TR, Fujiwara PI, Washko RM, Hamburg MA. Tuberculosis in New York City—turning the tide. N Engl J Med 1995; 333: 229-33.

105 Comstock GW, Philip RN. Decline of the tuberculosis epidemic in Alaska. Public Health Rep 1961; 76: 19-24.

106 Raviglione M, Uplekar M, Weil D, Kasaeva T. Tuberculosis makes it onto the international political agenda for health...finally. Lancet Glob Health 2018; 6: e20-21.

107 Shete PB, Reid M, Goosby E. Message to world leaders: we cannot end tuberculosis without addressing the social and economic burden of the disease. Lancet Glob Health 2018; 6: e1272-73.

108 Shimao T. [Tuberculosis prevalence survey in Japan]. Kekkaku 2009; 84: 713-20.

109 Zhu S, Xia L, Yu S, Chen S, Zhang J. The burden and challenges of tuberculosis in China: findings from the Global Burden of Disease Study 2015. Sci Rep 2017; 7: 14601.

110 Wang L, Zhang H, Ruan Y, et al. Tuberculosis prevalence in China, 1990-2010; a longitudinal analysis of national survey data. Lancet 2014; 383: 2057-64.

111 Suárez PG, Watt CJ, Alarcón E, et al. The dynamics of tuberculosis in response to 10 years of intensive control effort in Peru. $J$ Infect Dis 2001; 184: 473-78.

112 Fofana MO, Knight GM, Gomez GB, White RG, Dowdy DW. Population-level impact of shorter-course regimens for tuberculosis: a model-based analysis. PLoS One 2014; 9: e96389.

113 Jamison DT, Murphy SM, Sandbu ME. Why has under-5 mortality decreased at such different rates in different countries? J Health Econ 2016; 48: 16-25.

114 Jamison DT, Summers LH, Alleyne G, et al. Global health 2035: a world converging within a generation. Lancet 2013; 382: 1898-955.

115 Odone A, Roberts B, Dara M, van den Boom M, Kluge H, McKee M. People- and person-centred care for tuberculosis: models of care for tuberculosis. Int J Tuberc Lung Dis 2018; 22: 133-38. 
116 Seddon JA, Graham SM. Childhood TB: can the End TB Strategy deliver? Trans R Soc Trop Med Hyg 2016; 110: 155-57.

117 Kruk ME, Yamey G, Angell SY, et al. Transforming global health by improving the science of scale-up. PLoS Biol 2016; 14: e1002360.

118 Ali SM, Naureen F, Noor A, et al. Loss-to-follow-up and delay to treatment initiation in Pakistan's national tuberculosis control programme. BMC Public Health 2018; 18: 335.

119 Asres M, Gedefaw M, Kahsay A, Weldu Y. Patients' delay in seeking health care for tuberculosis diagnosis in East Gojjam zone, Northwest Ethiopia. Am J Trop Med Hyg 2017; 96: 1071-75.

120 Dye C. Expanded health systems for sustainable development. Science 2018; 359: 1337-39.

121 Gelmanova IY, Taran DV, Mishustin SP, Golubkov AA, Solovyova AV, Keshavjee S. 'Sputnik': a programmatic approach to improve tuberculosis treatment adherence and outcome among defaulters. Int J Tuberc Lung Dis 2011; 15: 1373-79.

122 Patient Pathway Analysis: how-to guide: Linksbridge. 2017. https://linksbridge.com/wp-content/uploads/2017/10/TB_PatientPathways-Guide.pdf (accessed Feb 27, 2019).

123 WHO. Report of the 16th Meeting of the Strategic and Technical Advisory Group for Tuberculosis. Geneva: World Health Organization, 2016.

124 Trollip A, Albert H, Purcell R, et al. TB Diagnostic Network Optimization-a key tool to inform improved access to TB diagnosis and treatment. 5th South African TB Conference; Durban, South Africa; June 12-15, 2018.

125 Wyber R, Vaillancourt S, Perry W, Mannava P, Folaranmi T, Celi LA. Big data in global health: improving health in low- and middle-income countries. Bull World Health Organ 2015; 93: 203-08.

126 Ocampo JMF, Smart JC, Allston A, et al. Improving HIV surveillance data for public health action in Washington, DC: a novel multiorganizational data-sharing method. JMIR Public Health Surveill 2016; 2: e3.

127 Miranda ML, Ferranti J, Strauss B, Neelon B, Califf RM. Geographic health information systems: a platform to support the 'triple aim'. Health Aff (Millwood) 2013; 32: 1608-15.

128 Balicer RD, Luengo-Oroz M, Cohen-Stavi C, et al. Using big data for non-communicable disease surveillance. Lancet Diabetes Endocrinol 2018; 6: 595-98.

129 Orellana-Presentacion M, Benavides-Luyo C, Taype-Rondan A. [Support groups for patients with tuberculosis in Facebook] Rev Med Chil 2016; 144: 819.

130 Hermans SM, Elbireer S, Tibakabikoba H, Hoefman BJ, Manabe YC. Text messaging to decrease tuberculosis treatment attrition in TB-HIV coinfection in Uganda. Patient Prefer Adherence 2017; 11: 1479-87.

131 Mohammed S, Glennerster R, Khan AJ. Impact of a daily SMS medication reminder system on tuberculosis treatment outcomes: a randomized controlled Trial. PLoS One 2016; 11: e0162944.

132 Small PM. Smartphones should fuel the next generation of tuberculosis care. 2018. https://www.statnews.com/2018/10/23/ smartphones-next-generation-tuberculosis-care/ (accessed Dec 15, 2018).

133 Satyanarayana S, Subbaraman R, Shete P, et al. Quality of tuberculosis care in India: a systematic review. Int $J$ Tuberc Lung Dis 2015; 19: 751-63.

134 Bardfield J, Palumbo M, Geis M, Jasmin M, Agins BD, and the NOA Working Group. A National Organizational Assessment (NOA) to build sustainable quality management programs in low- and middle- income countries. Jt Comm J Qual Patient Saf 2016; 42: 325-30.

135 Leatherman S, Ferris TG, Berwick D, Omaswa F, Crisp N. The role of quality improvement in strengthening health systems in developing countries. Int J Qual Health Care 2010; 22: 237-43.

136 McCarthy EA, Subramaniam HL, Prust ML, et al. Quality improvement intervention to increase adherence to ART prescription policy at HIV treatment clinics in Lusaka, Zambia: a cluster randomized trial. PLoS One 2017; 12: e0175534.

137 Youngleson MS, Nkurunziza P, Jennings K, Arendse J, Mate KS, Barker P. Improving a mother to child HIV transmission programme through health system redesign: quality improvement, protocol adjustment and resource addition. PLoS One 2010; 5: e13891.
138 Lolekha R, Chunwimaleung S, Hansudewechakul R, et al. Pediatric HIVQUAL-T: measuring and improving the quality of pediatric HIV care in Thailand, 2005-2007. Jt Comm J Qual Patient Saf2010; 36: 541-51.

139 Hung A, Pradel F. A review of how the quality of HIV clinical services has been evaluated or improved. Int J STD AIDS 2015; 26: 445-55.

140 Bardfield J, Agins B, Akiyama M, et al. A quality improvement approach to capacity building in low- and middle-income countries. AIDS 2015; 29 (suppl 2): S179-86.

141 TB CARE I. International standards for tuberculosis care, edition 3. 2014. URL: https://www.who.int/tb/publications/ISTC_3rdEd.pdf (accessed Feb 27, 2019).

142 Vesga JF, Hallett TB, Reid MJA, et al. Assessing tuberculosis control priorities in high-burden settings: a modelling approach. Lancet Glob Health 2019; published online March 20. http://dx.doi.org/10.1016/S2214-109X(19)30037-3.

143 Reid MJ, Shah NS. Approaches to tuberculosis screening and diagnosis in people with HIV in resource-limited settings. Lancet Infect Dis 2009; 9: 173-84.

144 Law I, Sylavanh P, Bounmala S, et al. The first national tuberculosis prevalence survey of Lao PDR (2010-2011). Trop Med Int Health 2015; 20: 1146-54.

145 Onozaki I, Law I, Sismanidis C, Zignol M, Glaziou P, Floyd K. National tuberculosis prevalence surveys in Asia, 1990-2012: an overview of results and lessons learned. Trop Med Int Health 2015 20: $1128-45$.

146 Ayles H, Schaap A, Nota A, et al, and the Peter Godfrey-Faussett for the ZAMSTAR Study Team. Prevalence of tuberculosis, HIV and respiratory symptoms in two Zambian communities: implications for tuberculosis control in the era of HIV. PLoS One 2009; 4: e5602.

147 Getahun H, Kittikraisak W, Heilig CM, et al. Development of a standardized screening rule for tuberculosis in people living with HIV in resource-constrained settings: individual participant data meta-analysis of observational studies. PLoS Med 2011; 8: e1000391.

148 Fox GJ, Barry SE, Britton WJ, Marks GB. Contact investigation for tuberculosis: a systematic review and meta-analysis. Eur Respir J 2013; 41: 140-56.

149 Barboza CE, Winter DH, Seiscento M, Santos UP, Terra Filho M. Tuberculosis and silicosis: epidemiology, diagnosis and chemoprophylaxis. J Bras Pneumol 2008; 34: 959-66.

150 Baussano I, Williams BG, Nunn P, Beggiato M, Fedeli U, Scano F. Tuberculosis incidence in prisons: a systematic review. PLoS Med 2010; 7: e1000381.

151 Kranzer K, Houben RM, Glynn JR, Bekker LG, Wood R, Lawn SD. Yield of HIV-associated tuberculosis during intensified case finding in resource-limited settings: a systematic review and meta-analysis. Lancet Infect Dis 2010; 10: 93-102.

152 Ayles H, Muyoyeta M, Du Toit E, et al, and the ZAMSTAR team. Effect of household and community interventions on the burden of tuberculosis in southern Africa: the ZAMSTAR community-randomised trial. Lancet 2013; 382: 1183-94.

153 Cavalcante SC, Durovni B, Barnes GL, et al. Community-randomized trial of enhanced DOTS for tuberculosis control in Rio de Janeiro, Brazil. Int J Tuberc Lung Dis 2010; 14: 203-09.

154 Corbett EL, Bandason T, Duong T, et al. Comparison of two active case-finding strategies for community-based diagnosis of symptomatic smear-positive tuberculosis and control of infectious tuberculosis in Harare, Zimbabwe (DETECTB): a cluster-randomised trial. Lancet 2010; 376: 1244-53.

155 Okada K, Onozaki I, Yamada N, et al. Epidemiological impact of mass tuberculosis screening: a 2-year follow-up after a national prevalence survey. Int J Tuberc Lung Dis 2012; 16: 1619-24.

156 Fox GJ, Nhung NV, Sy DN, et al. Household-Contact Investigation for Detection of Tuberculosis in Vietnam. N Engl J Med 2018; 378: 221-29.

157 Dowdy DW, Lotia I, Azman AS, Creswell J, Sahu S, Khan AJ. Population-level impact of active tuberculosis case finding in an Asian megacity. PLoS One 2013; 8: e77517.

158 Yuen CM, Amanullah F, Dharmadhikari A, et al. Turning off the tap: stopping tuberculosis transmission through active case-finding and prompt effective treatment. Lancet 2015; 386: 2334-43.

159 Uplekar M, Creswell J, Ottmani SE, Weil D, Sahu S, Lönnroth K. Programmatic approaches to screening for active tuberculosis. Int J Tuberc Lung Dis 2013; 17: 1248-56. 
160 Stop TB. Partnership U. GLOBAL PLAN TO END TB; revised funding estimates 2018-2022. Geneva: Stop TB Partnership, 2018.

161 Madon T, Hofman KJ, Kupfer L, Glass RI. Public health. Implementation science. Science 2007; 318: 1728-29.

162 Yamey G. Scaling up global health interventions: a proposed framework for success. PLoS Med 2011; 8: e1001049.

163 Fox GJ, Nhung NV, Sy DN, Britton WJ, Marks GB. Household contact investigation for tuberculosis in Vietnam: study protocol for a cluster randomized controlled trial. Trials 2013; 14: 342.

164 Citro B, Lyon E, Mankad M, Pandey KR, Gianella C. Developing a Human Rights-Based Approach to Tuberculosis. Health Hum Rights 2016; 18: $1-8$.

165 Li L, Lin Y, Mi F, et al. Screening of patients with tuberculosis for diabetes mellitus in China. Trop Med Int Health 2012; 17: 1294-301.

166 Khan AJ, Khowaja S, Khan FS, et al. Engaging the private sector to increase tuberculosis case detection: an impact evaluation study. Lancet Infect Dis 2012; 12: 608-16.

167 Datiko DG, Lindtjørn B. Health extension workers improve tuberculosis case detection and treatment success in southern Ethiopia: a community randomized trial. PLoS One 2009; 4: e5443.

168 Wingfield T, Tovar MA, Datta S, Saunders MJ, Evans CA. Addressing social determinants to end tuberculosis. Lancet 2018; 391: 1129-32.

169 Kranzer K, Lawn SD, Meyer-Rath G, et al. Feasibility, yield, and cost of active tuberculosis case finding linked to a mobile HIV service in Cape Town, South Africa: a cross-sectional study. PLoS Med 2012; 9: e1001281.

170 Mandalakas AM, Hesseling AC, Gie RP, Schaaf HS, Marais BJ, Sinanovic E. Modelling the cost-effectiveness of strategies to prevent tuberculosis in child contacts in a high-burden setting. Thorax 2013; 68: 247-55.

171 Azman AS, Golub JE, Dowdy DW. How much is tuberculosis screening worth? Estimating the value of active case finding for tuberculosis in South Africa, China, and India. BMC Med 2014; 12: 216.

172 Akachi Y, Zumla A, Atun R. Investing in improved performance of national tuberculosis programs reduces the tuberculosis burden: analysis of 22 high-burden countries, 2002-2009. J Infect Dis 2012 205 (suppl 2): S284-92.

173 Department of Health of South Africa. South African National AIDS Council: South African HIV and TB Investment Casesummary report phase 1. 2016. http://www.heroza.org/wp-content/ uploads/2016/03/SA-HIV_TB-Investment-Case-Full-Report-LowRes.pdf (accessed Feb 23, 2019).

174 WHO. ScreenTB - target prioritization and strategy selection for tuberculosis screening (active case finding). 2016. https://wpro. shinyapps.io/screen_tb/ (accessed April 18, 2018).

175 Melendez J, Sánchez CI, Philipsen RH, et al. An automated tuberculosis screening strategy combining X-ray-based computer-aided detection and clinical information. Sci Rep 2016; 6: 25265.

176 Philipsen RH, Sánchez CI, Maduskar P, et al. Automated chest-radiography as a triage for Xpert testing in resource-constrained settings: a prospective study of diagnostic accuracy and costs. Sci Rep 2015; 5: 12215.

177 Pande T, Cohen C, Pai M, Ahmad Khan F. Computer-aided detection of pulmonary tuberculosis on digital chest radiographs: a systematic review. Int J Tuberc Lung Dis 2016; 20: 1226-30.

178 Brown LB, Ayieko J, Mwangwa F, et al. Predictors of retention in HIV care among youth (15-24) in a universal test-and-treat setting in Rural Kenya. J Acquir Immune Defic Syndr 2017; 76: e15-18.

179 Wesolowski A, Eagle N, Tatem AJ, et al. Quantifying the impact of human mobility on malaria. Science 2012; 338: 267-70.

180 WHO. Systematic Screening for active tubeculosis; principles and recommendations. Geneva, 2013.

181 Lönnroth K, Corbett E, Golub J, et al. Systematic screening for active tuberculosis: rationale, definitions and key considerations. Int J Tuberc Lung Dis 2013; 17: 289-98.

182 UNAIDS. HIV infections (all ages). 2018. http://aidsinfo.unaids.org (accessed Jan 31, 2019).

183 Dodd PJ, Gardiner E, Coghlan R, Seddon JA. Burden of childhood tuberculosis in 22 high-burden countries: a mathematical modelling study. Lancet Glob Health 2014; 2: e453-59.
184 Morrison J, Pai M, Hopewell PC. Tuberculosis and latent tuberculosis infection in close contacts of people with pulmonary tuberculosis in low-income and middle-income countries: a systematic review and meta-analysis. Lancet Infect Dis 2008; 8: 359-68.

185 Rangaka MX, Cavalcante SC, Marais BJ, et al. Controlling the seedbeds of tuberculosis: diagnosis and treatment of tuberculosis infection. Lancet 2015; 386: 2344-53.

186 WHO. WHO Policy on TB Infection Control in Health-Care Facilities, Congregate Settings and Households. Geneva, Switzerland, 2009.

187 Bock NN, Jensen PA, Miller B, Nardell E. Tuberculosis infection control in resource-limited settings in the era of expanding HIV care and treatment. J Infect Dis 2007; 196 (suppl 1): S108-13.

188 Reid MJ, Saito S, Nash D, Scardigli A, Casalini C, Howard AA. Implementation of tuberculosis infection control measures at HIV care and treatment sites in sub-Saharan Africa. Int $J$ Tuberc Lung Dis 2012; 16: 1605-12.

189 Snider DE Jr. The relationship between tuberculosis and silicosis. Am Rev Respir Dis 1978; 118: 455-60.

190 Samandari T, Agizew TB, Nyirenda S, et al. 6-month versus 36-month isoniazid preventive treatment for tuberculosis in adults with HIV infection in Botswana: a randomised, double-blind, placebo-controlled trial. Lancet 2011; 377: 1588-98.

191 Suthar AB, Lawn SD, del Amo J, et al. Antiretroviral therapy for prevention of tuberculosis in adults with HIV: a systematic review and meta-analysis. PLoS Med 2012; 9: e1001270.

192 Badje A, Moh R, Gabillard D, et al, and the Temprano ANRS 12136 Study Group. Effect of isoniazid preventive therapy on risk of death in west African, HIV-infected adults with high CD4 cell counts: long-term follow-up of the Temprano ANRS 12136 trial Lancet Glob Health 2017; 5: e1080-89.

193 Hakim J, Musiime V, Szubert AJ, et al, and the REALITY Trial Team. Enhanced Prophylaxis plus antiretroviral Therapy for Advanced HIV Infection in Africa. N Engl J Med 2017; 377: 233-45.

194 Dye C, Glaziou P, Floyd K, Raviglione M. Prospects for tuberculosis elimination. Annu Rev Public Health 2013; 34: 271-86.

195 Dodd PJ, Yuen CM, Becerra MC, Revill P, Jenkins HE, Seddon JA Potential effect of household contact management on childhood tuberculosis: a mathematical modelling study. Lancet Glob Health 2018; 6: e1329-38.

196 Balcells ME, Thomas SL, Godfrey-Faussett P, Grant AD. Isoniazid preventive therapy and risk for resistant tuberculosis. Emerg Infect Dis 2006; 12: 744-51.

197 Holland DP, Sanders GD, Hamilton CD, Stout JE. Costs and cost-effectiveness of four treatment regimens for latent tuberculosis infection. Am J Respir Crit Care Med 2009; 179: 1055-60.

198 Sterling TR, Villarino ME, Borisov AS, et al. Three months of rifapentine and isoniazid for latent tuberculosis infection. N Engl J Med 2011; 365: 2155-66.

199 Swindells S, Ramchandani R, Gupta A, et al. One month of rifapentine and isoniazid to prevent HIV-related tuberculosis. $N$ Engl J Med (in press).

200 Huang YW, Yang SF, Yeh YP, Tsao TC, Tsao SM. Impacts of 12-dose regimen for latent tuberculosis infection: Treatment completion rate and cost-effectiveness in Taiwan. Medicine (Baltimore) 2016; 95: e4126.

201 Shepardson D, Marks SM, Chesson H, et al. Cost-effectiveness of a 12-dose regimen for treating latent tuberculous infection in the United States. Int J Tuberc Lung Dis 2013; 17: 1531-37.

202 Chaisson RE, Golub JE. Preventing tuberculosis in people with HIV-no more excuses. Lancet Glob Health 2017; 5: e1048-49.

203 WHO. Latent TB Infection: Updated and consolidated guidelines for programmatic management. Geneva: World Health Organization, 2018.

204 Yuen CM, Jenkins HE, Chang R, Mpunga J, Becerra MC. Two methods for setting child-focused tuberculosis care targets. Public Health Action 2016; 6: 83-96.

205 Theron G, Jenkins HE, Cobelens F, et al. Data for action: collection and use of local data to end tuberculosis. Lancet 2015; 386: 2324-33.

206 Uplekar MW, Shepard DS. Treatment of tuberculosis by private general practitioners in India. Tubercle 1991; 72: 284-90. 
207 Lal SS, Uplekar M, Katz I, et al. Global Fund financing of public-private mix approaches for delivery of tuberculosis care. Trop Med Int Health 2011; 16: 685-92.

208 WHO. Joint Monitoring Mission: Revised National Tuberculosis Control Programme (RNTCP) India, 2009.

209 WHO. Involving private practitioners in tuberculosis control: issues, interventions and emerging policy framework. Geneva: World Health Organization, 2001

$210 \mathrm{Xu}$ K, Evans DB, Carrin G, Aguilar-Rivera AM, Musgrove P, Evans T. Protecting households from catastrophic health spending. Health Aff (Millwood) 2007; 26: 972-83.

211 Xu K, Evans DB, Kawabata K, Zeramdini R, Klavus J, Murray CJ. Household catastrophic health expenditure: a multicountry analysis. Lancet 2003; 362: 111-17.

212 Wells WA, Uplekar M, Pai M. Achieving systemic and scalable private sector engagement in tuberculosis care and prevention in Asia. PLoS Med 2015; 12: e1001842.

213 Lönnroth K, Uplekar M, Blanc L. Hard gains through soft contracts: productive engagement of private providers in tuberculosis control. Bull World Health Organ 2006; 84: 876-83.

214 Malmborg R, Mann G, Squire SB. A systematic assessment of the concept and practice of public-private mix for tuberculosis care and control. Int J Equity Health 2011; 10: 49.

215 Lei X, Liu Q, Escobar E, et al. Public-private mix for tuberculosis care and control: a systematic review. Int J Infect Dis 2015; 34: 20-32.

216 Floyd K, Arora VK, Murthy KJ, et al. Cost and cost-effectiveness of PPM-DOTS for tuberculosis control: evidence from India. Bull World Health Organ 2006; 84: 437-45.

217 Hanson CL, Osberg M, Brown J, Durham G, Chin DP. Conducting Patient-Pathway Analysis to Inform Programming of Tuberculosis Services: Methods. J Infect Dis 2017; 216 (suppl 7): S679-85

218 Wells WA, Ge CF, Patel N, Oh T, Gardiner E, Kimerling ME. Size and usage patterns of private TB drug markets in the high burden countries. PLoS One 2011; 6: e18964.

219 Wells WA, Stallworthy G, Balsara Z. How tuberculosis programs can navigate the world of social health insurance. Int J Tuberc Lung Dis 2019; 23: 26-37.

220 Caslyn M. Alternatives to Fee-for-Service payments in health care. 2013. https://www.americanprogress.org/issues/healthcare/ reports/2012/09/18/38320/alternatives-to-fee-for-service-paymentsin-health-care/ (accessed Dec 4, 2018).

221 Arinaminpathy N. Predicted impact of effective private provider engagement on tuberculosis control in urban India.

222 Kendall EA, Fofana MO, Dowdy DW. Burden of transmitted multidrug resistance in epidemics of tuberculosis: a transmission modelling analysis. Lancet Respir Med 2015; 3: 963-72.

223 O'Neill J. Review on antimicrobial resistance. Tackling drug-resistant infections globally: final report and reccomendations Wellcome Trust ad UK Government, 2016.

224 Lunte K, Cordier-Lassalle T, Keravec J. Reducing the price of treatment for multidrug-resistant tuberculosis through the Global Drug Facility. Bull World Health Organ 2015; 93: 279-82.

225 Medecins Sans Frontieres Partners in Health and the Treatment Action Group. An evaluation of drug-resistant TB treatment scale up. 2011.

226 Cobelens FG, Heldal E, Kimerling ME, et al. Scaling up programmatic management of drug-resistant tuberculosis: a prioritized research agenda. PLoS Med 2008; 5: e150.

227 WHO. Towards universal access to diagnosis and treatment of multidrug-resistant and extensively drug-resistant tuberculosis by 2015: WHO progress report 2011. Geneva: World Health Organization, 2011

228 Hwang TJ, Keshavjee S. Global financing and long-term technical assistance for multidrug-resistant tuberculosis: scaling up access to treatment. PLoS Med 2014; 11: e1001738.

229 Ragonnet R, Trauer JM, Denholm JT, Marais BJ, McBryde ES High rates of multidrug-resistant and rifampicin-resistant tuberculosis among re-treatment cases: where do they come from? $B M C$ Infect Dis 2017; 17: 36.

230 Bloemberg GV, Keller PM, Stucki D, et al. Acquired resistance to bedaquiline and delamanid in therapy for tuberculosis. N Engl J Med 2015; 373: 1986-88.
231 Law S, Piatek AS, Vincent C, Oxlade O, Menzies D. Emergence of drug resistance in patients with tuberculosis cared for by the Indian health-care system: a dynamic modelling study. Lancet Public Health 2017; 2: e47-55.

232 Mitchison DA. How drug resistance emerges as a result of poor compliance during short course chemotherapy for tuberculosis. Int J Tuberc Lung Dis 1998; 2: 10-15.

233 Srivastava S, Pasipanodya JG, Meek C, Leff R, Gumbo T Multidrug-resistant tuberculosis not due to noncompliance but to between-patient pharmacokinetic variability. J Infect Dis 2011; 204: 1951-59.

234 March F, Garriga X, Rodriguez P, et al. Acquired drug resistance in Mycobacterium tuberculosis isolates recovered from compliant patients with human immunodeficiency virus-associated tuberculosis. Clin Infect Dis 1997; 25: 1044-47.

235 Zhao P, Li XJ, Zhang SF, Wang XS, Liu CY. Social behaviour risk factors for drug resistant tuberculosis in mainland China: a meta-analysis. J Int Med Res 2012; 40: 436-45.

236 van Ingen J, Aarnoutse RE, Donald PR, et al. Why do we use $600 \mathrm{mg}$ of rifampicin in tuberculosis treatment? Clin Infect Dis 2011; 52: e194-99.

237 Liang L, Wu Q, Gao L, et al. Factors contributing to the high prevalence of multidrug-resistant tuberculosis: a study from China. Thorax 2012; 67: 632-38.

238 Khan MS, Hutchison C, Coker RJ. Risk factors that may be driving the emergence of drug resistance in tuberculosis patients treated in Yangon, Myanmar. PLoS One 2017; 12: e0177999.

239 WHO. Good practices in strengthening health systems for the prevention and care of tuberculosis and drug-resistant tuberculosis: WHO Regional Office for Europe, 2016. Geneva: World Health Organization, 2016.

240 Colijn C, Cohen T, Ganesh A, Murray M. Spontaneous emergence of multiple drug resistance in tuberculosis before and during therapy. PLoS One 2011; 6: e18327.

241 Mitchison DA, Nunn AJ. Influence of initial drug resistance on the response to short-course chemotherapy of pulmonary tuberculosis. Am Rev Respir Dis 1986; 133: 423-30.

242 Shrestha S, Knight GM, Fofana M, et al. Drivers and trajectories of resistance to new first-line drug regimens for tuberculosis. Open Forum Infect Dis 2014; 1: ofu073.

243 Cegielski JP, Dalton T, Yagui M, et al. Extensive drug resistance acquired during treatment of multidrug-resistant tuberculosis. Clin Infect Dis 2014; 59: 1049-63.

244 Seddon JA, Warren RM, Enarson DA, Beyers N, Schaaf HS Drug-resistant tuberculosis transmission and resistance amplification within families. Emerg Infect Dis 2012; 18: 1342-45.

245 Temple B, Ayakaka I, Ogwang S, et al. Rate and amplification of drug resistance among previously-treated patients with tuberculosis in Kampala, Uganda. Clin Infect Dis 2008; 47: 1126-34.

246 Furin JJ, Becerra MC, Shin SS, Kim JY, Bayona J, Farmer PE. Effect of administering short-course, standardized regimens in individuals infected with drug-resistant Mycobacterium tuberculosis strains. Eur J Clin Microbiol Infect Dis 2000; 19: 132-36.

247 Prabhu BP, Kunoor A, Sudhir S, et al. Anti-tuberculosis treatment stewardship in a private tertiary care hospital in South India. Public Health Action 2018; 8: 151.

248 Pai M, Schito M. Tuberculosis diagnostics in 2015: landscape, priorities, needs, and prospects. J Infect Dis 2015; 211 (suppl 2): S21-28.

249 Boehme CC, Nicol MP, Nabeta P, et al. Feasibility, diagnostic accuracy, and effectiveness of decentralised use of the Xpert MTB/RIF test for diagnosis of tuberculosis and multidrug resistance: a multicentre implementation study. Lancet 2011; 377: 1495-505.

250 Tomasicchio M, Theron G, Pietersen E, et al. The diagnostic accuracy of the MTBDRplus and MTBDRsl assays for drug-resistant TB detection when performed on sputum and culture isolates. Sci Rep 2016; 6: 17850.

251 Albert H, Bwanga F, Mukkada S, et al. Rapid screening of MDR-TB using molecular Line Probe Assay is feasible in Uganda. BMC Infect Dis 2010; 10: 41.

252 Cox H, Dickson-Hall L, Ndjeka N, et al. Delays and loss to follow-up before treatment of drug-resistant tuberculosis following implementation of Xpert MTB/RIF in South Africa: a retrospective cohort study. PLoS Med 2017; 14: e1002238. 
253 Boyd R, Ford N, Padgen P, Cox H. Time to treatment for rifampicin-resistant tuberculosis: systematic review and meta-analysis. Int J Tuberc Lung Dis 2017; 21: 1173-80.

254 Chakravorty S, Simmons AM, Rowneki M, et al. The new Xpert MTB/RIF Ultra: improving detection of Mycobacterium tuberculosis and resistance to rifampin in an assay suitable for point-of-care testing. MBio 2017; 8 (4): pii: e00812-17.

255 Xie YL, Chakravorty S, Armstrong DT, et al. Evaluation of a rapid molecular drug-susceptibility test for tuberculosis. $N$ Engl J Med 2017; 377: 1043-54.

256 Ifakara Health Institute. Evaluation of CEPHEId GeneXpert Omni combined with the Xpert MTB/RIF Ultra in primary health care in Tanzania. 2018. https://ihi.or.tz/project/cepheid-genexpert-omnicombined-with-the-xpert-mtbrif-ultra-for-detection-of-tuberculosisand-rifampicin-resistance-in-adults-with-presumptive-pulmonarytuberculosis-at-primary-level-diagnostic-cent/ (accessed Jan 16, 2018).

257 Lessells RJ, Cooke GS, McGrath N, Nicol MP, Newell ML, Godfrey-Faussett P. Impact of point-of-care Xpert MTB/RIF on tuberculosis treatment initiation: a cluster randomised trial. Am J Respir Crit Care Med 2017; 196: 901-10.

258 Hanrahan CF, Clouse K, Bassett J, et al. The patient impact of point-of-care vs. laboratory placement of Xpert((R)) MTB/RIF Int J Tuberc Lung Dis 2015; 19: 811-16.

259 Ahuja SD, Ashkin D, Avendano M, et al. Multidrug resistant pulmonary tuberculosis treatment regimens and patient outcomes: an individual patient data meta-analysis of 9,153 patients. PLoS Med 2012; 9: e1001300.

260 Wu S, Zhang Y, Sun F, et al. Adverse events associated with the treatment of multidrug-resistant tuberculosis: a systematic review and meta-analysis. Am J Ther 2016; 23: e521-30.

261 Shringarpure KS, Isaakidis P, Sagili KD, Baxi RK, Das M, Daftary A "When treatment is more challenging than the disease": a qualitative study of MDR-TB patient retention. PLoS One 2016; 11: e0150849.

262 Brigden G, Nyang'wa BT, du Cros P, et al. Principles for designing future regimens for multidrug-resistant tuberculosis. Bull World Health Organ 2014; 92: 68-74.

263 WHO. WHO treatment guidelines for drug-resistant tuberculosis: 2016 update. Geneva: World Health Organization, 2016

264 WHO. The use of delamanid in the treatment of multidrug-resistant tuberculosis: interim policy guidance. Geneva: World Health Organization, 2014.

265 WHO. The use of bedaquiline in the treatment of multidrug-resistant tuberculosis: World Health Organization, 2013.

266 RESIST-TB (Research Excellence to Stop TB Resistance). Drug-resistant tuberculosis clinical trials progress report. 2017. http://www.resisttb.org/wp-content/uploads/2018/11/RESIST-TBCTPR_11_17_2018.pdf (accessed Feb 24, 2019).

267 Cox HS, Furin JJ, Mitnick CD, Daniels C, Cox V, Goemaere E. The need to accelerate access to new drugs for multidrug-resistant tuberculosis. Bull World Health Organ 2015; 93: 491-97.

268 WHO. Report of the Guideline Development Group Meeting on the use of bedaquiline in the treatment of multidrug-resistant tuberculosis, a review of available evidence (2016). Geneva: World Health Organization, 2016.

269 Interim clinical guidance for the implementation of injectable-free regimens for rifampicin-resistant tuberculosis in adults, adolescents and children. Pretoria: National Department of Health South Africa. 2018.

270 WHO. Rapid communication: key changes to treatment of multidrug- and rifampicin-resistant tuberculosis (MDR/RR-TB). Genevaa: World Health Organization, 2018.

271 Seddon JA, Godfrey-Faussett P, Jacobs K, Ebrahim A, Hesseling AC Schaaf HS. Hearing loss in patients on treatment for drug-resistant tuberculosis. Eur Respir J 2012; 40: 1277-86.

272 Reuter A, Tisile P, von Delft D, et al. The devil we know: is the use of injectable agents for the treatment of MDR-TB justified? Int J Tuberc Lung Dis 2017; 21: 1114-26.

273 Cox V, Brigden G, Crespo RH, et al. Global programmatic use of bedaquiline and delamanid for the treatment of multidrug-resistant tuberculosis. Int J Tuberc Lung Dis 2018; 22: 407-12.

274 Furin J, Brigden G, Lessem E, Rich M, Vaughan L, Lynch S. Global progress and challenges in implementing new medications for treating multidrug-resistant tuberculosis. Emerg Infect Dis 2016; published online March. DOI:10.3201/eid2203.151430.
275 Cox H, Hughes J, Black J, Nicol MP. Precision medicine for drug-resistant tuberculosis in high-burden countries: is individualised treatment desirable and feasible? Lancet Infect Dis 2018; 18: e282-87.

276 WHO. Active tuberculosis drug-safety monitoring and management (aDSM): framework for implementation. Geneva: World Health Organization, 2015.

277 Tanani DS, Serragui S, Hammi S, et al. National strategy for the integration of pharmacovigilance in the Moroccan TB Control Program. Pan Afr Med J 2017; 26: 48.

278 Nahid P. CURE-TB strategy stratified medicine trials. 2018 http://tb.ucsf.edu/tb-therapeutics-trials-pharmacokinetics (accessed Dec 8, 2018)

279 Toczek A, Cox H, du Cros P, Cooke G, Ford N. Strategies for reducing treatment default in drug-resistant tuberculosis: systematic review and meta-analysis. Int J Tuberc Lung Dis 2013; 17: 299-307.

280 Cox H, Ford N. Decentralisation of multidrug-resistant-tuberculosis care and management. Lancet Infect Dis 2013; 13: 644-46.

281 WHO. Latent tuberculosis infection: updated and consolidated guidelines for programmatic management. Geneva: World Health Organization, 2018

282 Laurence YV, Griffiths UK, Vassall A. Costs to health services and the patient of treating tuberculosis: a systematic literature review. Pharmacoeconomics 2015; 33: 939-55.

283 Oeltmann JE, Varma JK, Ortega L, et al. Multidrug-resistant tuberculosis outbreak among US-bound Hmong refugees, Thailand, 2005. Emerg Infect Dis 2008; 14: 1715-21.

284 Global health security agenda. Implementing the global health security agenda: progress and impact from US Government Investments. 2018. https://www.ghsagenda.org/docs/default-source/ default-document-library/global-health-security-agenda-2017-progressand-impact-from-u-s-investments.pdf?sfvrsn=4 (accessed Feb 24, 2019)

285 Paulson T. Epidemiology: a mortal foe. Nature 2013; 502: S2-3.

286 Bloom BR, Rifat Atun, Ted Cohen, et al. Tuberculosis. In: Jamison DT, Jha P, eds. Disease control priorities in developing countries, 3rd edition. Washington, DC: Oxford University Press, 2017: 233-93.

287 Petruccioli E, Scriba TJ, Petrone L, et al. Correlates of tuberculosis risk: predictive biomarkers for progression to active tuberculosis. Eur Respir J 2016; 48: 1751-63.

288 Marais BJ, Schaaf HS. Childhood tuberculosis: an emerging and previously neglected problem. Infect Dis Clin North Am 2010; 24: 727-49.

289 Zawedde-Muyanja S, Nakanwagi A, Dongo JP, et al. Decentralisation of child tuberculosis services increases case finding and uptake of preventive therapy in Uganda. Int J Tuberc Lung Dis 2018; 22: 1314-21.

290 Jaganath D, Mupere E. Childhood tuberculosis and malnutrition. J Infect Dis 2012; 206: 1809-15.

291 Dodd PJ, Prendergast AJ, Beecroft C, Kampmann B, Seddon JA. The impact of HIV and antiretroviral therapy on TB risk in children a systematic review and meta-analysis. Thorax 2017; 72: 559-75.

292 Pawlowski A, Jansson M, Skold M, Rottenberg ME, Kallenius G. Tuberculosis and HIV co-infection. PLoS pathogens 2012; 8: e1002464.

293 Fauci AS, Eisinger RW. Reimagining the research approach to tuberculosis. Am J Trop Med Hyg 2018; 98: 650-52.

294 Flynn JL, Chan J. Tuberculosis: latency and reactivation. Infect Immun 2001; 69: 4195-201.

295 Zak DE, Penn-Nicholson A, Scriba TJ, et al. A blood RNA signature for tuberculosis disease risk: a prospective cohort study. Lancet 2016; 387: 2312-22.

296 NIAID. TB Strategic Plan. 2018. https://www.niaid.nih.gov/sites/ default/files/TBStrategicPlan2018.pdf (accessed Nov 12, 2018).

297 Fauci AS. Addressing the tuberculosis epidemic: 21st century research for an ancient disease. JAMA 2018; 320: 1315-16.

298 Pai M, Nicol MP, Boehme CC. Tuberculosis diagnostics: state of the art and future directions. Microbiol Spectr 2016; 4. DOI:10.1128/ microbiolspec.TBTB2-0019-2016.

299 Frick M. The ascent begins: tuberculosis research funding trends, 2005-2016. 2017. http://treatmentactiongroup.org/sites/default/ files/TB_FUNDING_2017_final.pdf (accessed Feb 24, 2019). 
300 Kik SV, Denkinger CM, Casenghi M, Vadnais C, Pai M. Tuberculosis diagnostics: which target product profiles should be prioritised? Eur Respir J 2014; 44: 537-40.

301 Sun AY, Pai M, Salje H, Satyanarayana S, Deo S, Dowdy DW. Modeling the impact of alternative strategies for rapid molecular diagnosis of tuberculosis in Southeast Asia. Am J Epidemiol 2013; 178: $1740-49$

302 Bastos ML, Hussain H, Weyer K, et al. Treatment outcomes of patients with multidrug-resistant and extensively drug-resistant tuberculosis according to drug susceptibility testing to first- and second-line drugs: an individual patient data meta-analysis. Clin Infect Dis 2014; 59: 1364-74.

303 WHO. WHO treatment guidelines for isoniazid-resistant tuberculosis supplement to the WHO treatment guidelines for drug-resistant tuberculosis. Geneva: World Health Organization, 2018.

304 Sweeney TE, Braviak L, Tato CM, Khatri P. Genome-wide expression for diagnosis of pulmonary tuberculosis: a multicohort analysis. Lancet Respir Med 2016; 4: 213-24.

305 De Groote MA, Sterling DG, Hraha T, et al. Discovery and validation of a six-marker serum protein signature for the diagnosis of active pulmonary tuberculosis. J Clin Microbiol 2017; 55: 3057-71.

306 Pankhurst LJ, Del Ojo Elias C, Votintseva AA, et al. Rapid, comprehensive, and affordable mycobacterial diagnosis with whole-genome sequencing: a prospective study. Lancet Respir Med 2016; 4: 49-58

307 Walker TM, Kohl TA, Omar SV, et al. Whole-genome sequencing for prediction of Mycobacterium tuberculosis drug susceptibility and resistance: a retrospective cohort study. Lancet Infect Dis 2015; 15: 1193-202.

308 Gardiner JL, Karp CL. Transformative tools for tackling tuberculosis. $J$ Exp Med 2015; 212: 1759-69.

309 WHO. Target regimen profiles for TB treatment: candidates: rifampicin-susceptible, rifampicin-resistant and pan-TB treatment regimens. Geneva: World Health Organization, 2016.

310 Jenkins HE. Global burden of childhood tuberculosis. Pneumonia (Nathan) 2016; 8: 24.

311 Lienhardt C, Lonnroth K, Menzies D, et al. Translational research for tuberculosis elimination: priorities, challenges, and actions. PLoS Med 2016; 13: e1001965.

312 Jindani A, Harrison TS, Nunn AJ, et al. High-dose rifapentine with moxifloxacin for pulmonary tuberculosis. N Engl J Med 2014; 371: 1599-608.

313 Gillespie SH, Crook AM, McHugh TD, et al. Four-month moxifloxacin-based regimens for drug-sensitive tuberculosis. N Engl J Med 2014; 371: 1577-87.

314 Merle CS, Fielding K, Sow OB, et al. A four-month gatifloxacin-containing regimen for treating tuberculosis N Engl J Med 2014; 371: 1588-98.

315 Stop TB Partnership. 2017 Global new TB drug pipeline. 2017.

316 Wallis RS, Cohen T, Menzies NA, Churchyard G. Pan-tuberculosis regimens: an argument for. Lancet Respir Med 2018; 6: 239-40.

317 Dheda K, Gumbo T, Lange C, Horsburgh CR Jr, Furin J. Pan-tuberculosis regimens: an argument against. Lancet Respir Med 2018; 6: 240-42.

318 Roy A, Eisenhut M, Harris RJ, et al. Effect of BCG vaccination against Mycobacterium tuberculosis infection in children: systematic review and meta-analysis. BMJ 2014; 349: g4643.

319 Knight GM, Griffiths UK, Sumner T, et al. Impact and cost-effectiveness of new tuberculosis vaccines in low- and middle-income countries. PNAS 2014; 111: 15520-25.

320 von Reyn CF, Mtei L, Arbeit RD, et al. Prevention of tuberculosis in Bacille Calmette-Guerin-primed, HIV-infected adults boosted with an inactivated whole-cell mycobacterial vaccine. AIDS 2010; 24: 675-85.

321 Vordermeier HM, Villarreal-Ramos B, Cockle PJ, et al. Viral booster vaccines improve Mycobacterium bovis BCG-induced protection against bovine tuberculosis. Infect Immun 2009; 77: 3364-73.

322 Verreck FA, Vervenne RA, Kondova I, et al. MVA.85A boosting of BCG and an attenuated, phoP deficient $M$. tuberculosis vaccine both show protective efficacy against tuberculosis in rhesus macaques. PLoS One 2009; 4: e5264.

323 Bloom BR. New promise for vaccines against tuberculosis. N Engl J Med 2018; 379: 1672-74.
324 Nemes E, Geldenhuys H, Rozot V, et al. Prevention of M. tuberculosis infection with H4:IC31 vaccine or BCG revaccination. N Engl J Med 2018; 379: 138-49.

325 Van Der Meeren O, Hatherill M, Nduba V, et al. Phase 2b controlled trial of M72/AS01E vaccine to prevent tuberculosis. N Engl J Med 2018; 379: 1621-34.

326 WHO. Global investments in tuberculosis research and development past, present and future. A policy prepared for the first WHO global ministerial conference on ending tuberculosis in the sustainable development era: a multisectoral response. Geneva: World Health Organization, 2017.

327 Nguyen TA, Pham MT, Nguyen TL, et al. Video Directly Observed Therapy to support adherence with treatment for tuberculosis in Vietnam: a prospective cohort study. Int J Infect Dis 2017; 65: 85-89.

328 Liu X, Blaschke T, Thomas B, et al. Usability of a medication event reminder monitor system (MERM) by providers and patients to improve adherence in the management of tuberculosis. Int J Environ Res Public Health 2017; 14: pii: E1115.

329 Ha YP, Tesfalul MA, Littman-Quinn R, et al. Evaluation of a mobile health approach to tuberculosis contact tracing in Botswana. $J$ Health Commun 2016; 21: 1115-21.

330 Dwolatzky B, Trengove E, Struthers H, McIntyre JA, Martinson NA Linking the global positioning system (GPS) to a personal digital assistant (PDA) to support tuberculosis control in South Africa: a pilot study. Int J Health Geogr 2006; 5: 34.

331 Molton JS, Pang Y, Wang Z, et al. Prospective single-arm interventional pilot study to assess a smartphone-based system for measuring and supporting adherence to medication. BMJ Open 2016; 6: e014194.

332 Story A, Garfein RS, Hayward A, et al. Monitoring therapy compliance of tuberculosis patients by using video-enabled electronic devices. Emerg Infect Dis 2016; 22: 538-40.

333 Glaziou P, Floyd K, Korenromp EL, et al. Lives saved by tuberculosis control and prospects for achieving the 2015 global target for reducing tuberculosis mortality. Bull World Health Organ 2011; 89: 573-82.

334 Hammitt J, \& Robinson, L. The income elasticity of the value per statistical life: transferring estimates between high and low income populations. J Benefit-Cost Analysis 2011; 2: 1-29.

335 Lindhjem H, Navrud S, Braathen NA, Biausque V. Valuing mortality risk reductions from environmental, transport, and health policies: a global meta-analysis of stated preference studies. Risk Anal 2011; 31: 1381-407.

336 Mueller-Langer F. Neglected infectious diseases: are push and pull incentive mechanisms suitable for promoting drug development research? Health Econ Policy Law 2013; 8: 185-208.

337 Terry RF, Yamey G, Miyazaki-Krause R, Gunn A, Reeder JC. Funding global health product R\&D: the Portfolio-To-Impact Model (P2I), a new tool for modelling the impact of different research portfolios. Gates Open Res 2018; 2: 24.

338 Young R, Bekele T, Gunn A, et al. Developing new health technologies for neglected diseases: a pipeline portfolio review and cost model. Gates Open Res 2018, 2: 23.

339 Frick M. 2016 report on tuberculosis research funding trends, 20052015: no time to lose: treatment action group, 2016. http://www. treatmentactiongroup.org/sites/default/files/TB_FUNDING_2016 WEB.pdf (accessed Feb 24, 2019).

340 Saez C. WHO still finding its way on financing R\&D for diseases affecting poor countries. 2014. https://www.ip-watch org/2015/02/02/who-still-finding-its-way-on-financing-rd-fordiseases-affecting-poor-countries/ (accessed Feb 2, 2015).

341 Viergever RF. The mismatch between the health research and development $(R \& D)$ that is needed and the $R \& D$ that is undertaken: an overview of the problem, the causes, and solutions. Glob Health Action 2013; 6: 22450

342 Manufacturers IFoP. TB Drug Accelerator Program, 2017 http://www.cptrinitiative.org/wp-content/uploads/2017/05/ 20_01Berthel_CPTR_2017-03-21-v4.pdf (accessed Feb 24, 2019).

343 Slingsby BT, Kurokawa K. The Global Health Innovative Technology (GHIT) Fund: financing medical innovations for neglected populations. Lancet Glob Health 2013; 1: e184-85.

344 UNITAID. UNITAID ranked as one the leading funders of TB R\&D 2018. https://unitaid.org/news-blog/unitaid-ranked-as-one-of-theworlds-leaders-in-funding-tuberculosis-rd/\#en (accessed Aug 12, 2018). 
345 The economics of optimism. The Economist, (New York, NY), Jan 22, 2015.

346 Robinson LA, Hammitt, J.K., O'Keefe, L. Valuing mortality risk reductions in global benefit-cost analysis. 2018. https://cdn2.sph. harvard.edu/wp-content/uploads/sites/94/2017/09/RobinsonHammitt-OKeeffe-VSL-2017-10-27.pdf (accessed Feb 24, 2019).

347 Vassall A. Benefits and costs of the tuberculosis targets for the post-2015 development agenda. In: Lomborg, B. Prioritizing development: a cost benefit analysis of the United Nations' sustainable Development Goals. Cambridge: Cambridge University Press, 2014.

348 KPMG. Global economic impact of tuberculosis: KPMG, 2017.

349 Stop TB Partnership. The paradigm shift; 2016-2020—Global Plan to End TB. 2015. http://www.stoptb.org/assets/documents/global/ plan/globalplantoendtb_theparadigmshift_2016-2020_ stoptbpartnership.pdf (accessed date Feb 23, 2019).

350 Jamison, DT, Jha P, Laxminarayan R, et al. Infectious disease, injury and reproductive health. Expert panel ranking. In: Lomborg B, ed. Global problems, smart solutions: costs and benefits. Cambridge: Cambridge University Press, 2013: 701-16.

351 Kydland FER, Mundell R, Shelling T, Smith V, Bhagwati, J. Expert Panel Ranking. In: Lomborg B, ed. Global problems, smart solutions: costs and benefits. Cambridge: Cambridge University Press, 2013: 701-16.

352 Floyd K, Fitzpatrick C, Pantoja A, Raviglione M. Domestic and donor financing for tuberculosis care and control in low-income and middle-income countries: an analysis of trends, 2002-11, and requirements to meet 2015 targets. Lancet Glob Health 2013; 1: e105-15.

353 Fuady A, Houweling TAJ, Mansyur M, Richardus JH. Catastrophic total costs in tuberculosis-affected households and their determinants since Indonesia's implementation of universal health coverage. Infect Dis Poverty 2018; 7: 3.

354 Verguet S, Laxminarayan R, Jamison DT. Universal public finance of tuberculosis treatment in India: an extended cost-effectiveness analysis. Health Econ 2015; 24: 318-32.

355 Wingfield T, Boccia D, Tovar M, et al. Defining catastrophic costs and comparing their importance for adverse tuberculosis outcome with multi-drug resistance: a prospective cohort study, Peru. PLoS Med 2014; 11: e1001675.

356 Menzies NA, Gomez GB, Bozzani F, et al. Cost-effectiveness and resource implications of aggressive action on tuberculosis in China, India, and South Africa: a combined analysis of nine models. Lancet Glob Health 2016; 4: e816-26.

357 Ochalek J, Revill P, Manthalu G, et al. Supporting the development of a health benefits package in Malawi. BMJ Global Health 2018. 3: e000607.

358 Atun R, Weil DE, Eang MT, Mwakyusa D. Health-system strengthening and tuberculosis control. Lancet 2010; 375: 2169-78.

359 Sinanovic E, Ramma L, Vassall A, et al. Impact of reduced hospitalisation on the cost of treatment for drug-resistant tuberculosis in South Africa. Int J Tuberc Lung Dis 2015; 19: 172-78.

360 Atun R, de Jongh T, Secci F, Ohiri K, Adeyi O. A systematic review of the evidence on integration of targeted health interventions into health systems. Health Policy Plan 2010; 25: 1-14.

361 Cobelens F, van Kampen S, Ochodo E, Atun R, Lienhardt C. Research on implementation of interventions in tuberculosis control in low- and middle-income countries: a systematic review. PLoS Med 2012; 9: e1001358.

362 Carter DJ, Glaziou P, Lonnroth K, et al. The impact of social protection and poverty elimination on global tuberculosis incidence: a statistical modelling analysis of Sustainable Development Goal 1. Lancet Glob Health 2018; 6: e514-22.

363 Atun R, Silva S, Ncube M, Vassall A. Innovative financing for HIV response in sub-Saharan Africa. J Glob Health 2016; 6: 010407.

364 Pablos-Mendez A, Raviglione MC. A new world health era. Glob Health Sci Pract 2018; 6: 8-16.

365 IHME. Financing global health 2016: development assistance, public and private health spending, for the pursuit of Universal Health Coverage. Seattle, WA: Institute for Health Metrics and Evaluation, 2017.

366 Organization for Economic Co-operation and Development. Credit Report Scoring. https://stats.oecd.org/Index aspx? DataSetCode=CRS1 (accessed Feb 24, 2019).
367 Kumaresan J, Smith I, Arnold V, Evans P. The Global TB Drug Facility: innovative global procurement. Int J Tuberc Lung Dis 2004; 8: $130-38$

368 Arinaminpathy N, Cordier-Lassalle T, Vijay A, Dye C. The Global Drug Facility and its role in the market for tuberculosis drugs. Lancet 2013; 382: 1373-79.

369 Arinaminpathy N, Cordier-Lassalle T, Lunte K, Dye C. The Global Drug Facility as an intervention in the market for tuberculosis drugs. Bull World Health Organ 2015; 93: 237-48A.

370 Waning B. Tuberculosis Market-Shaping Strategy: Overview, Updates \& Priority Issues from Stop TB's Global Drug Facility. 2017; Global Drug Facility, Stop TB Partnership; 2017.

371 Stop TB Partnership. Challenge facility for civil society. 2018. http://www.stoptb.org/global/awards/cfcs/ (accessed April 25, 2018).

372 Getahun H, Raviglione M. Transforming the global tuberculosis response through effective engagement of civil society organizations: the role of the World Health Organization. Bull World Health Organ 2011; 89: 616-18.

373 Piot P, Abdool Karim SS, Hecht R, et al. Defeating AIDS-advancing global health. Lancet 2015; 386: 171-218.

374 Smith J, Mallouris C, Lee K, Alfven T. The role of civil society organizations in monitoring the global AIDS response. AIDS and behavior 2017; 21 (suppl 1): 44-50.

375 Lover AA, Harvard KE, Lindawson AE, et al. Regional initiatives for malaria elimination: Building and maintaining partnerships. PLoS Med 2017; 14: e1002401.

376 Mokrousov I. Molecular structure of Mycobacterium tuberculosis population in Russia and its interaction with neighboring countries. Int J Mycobacteriol 2015; 4 (suppl 1): 56-57.

377 Mokrousov I, Vyazovaya A, Solovieva N, et al. Trends in molecular epidemiology of drug-resistant tuberculosis in Republic of Karelia, Russian Federation. BMC Microbiol 2015; 15: 279

378 Zanini F, Carugati M, Schiroli C, et al. Mycobacterium tuberculosis Beijing family: analysis of the epidemiological and clinical factors associated with an emerging lineage in the urban area of Milan. Infect Genet Evol 2014; 25: 14-19.

379 Coscolla M, Barry PM, Oeltmann JE, et al. Genomic epidemiology of multidrug-resistant Mycobacterium tuberculosis during transcontinental spread. J Infect Dis 2015; 212: 302-10.

380 Yen S, Bower JE, Freeman JT, Basu I, O’Toole RF. Phylogenetic lineages of tuberculosis isolates in New Zealand and their association with patient demographics. Int J Tuberc Lung Dis 2013; 17: 892-97.

381 Vasankari T, Soini H, Liippo K, Ruutu P. MDR-TB in Finland-still rare despite the situation in our neighbouring countries. Clin Respir J 2012; 6: 35-39.

382 Rovina N, Karabela S, Constantoulakis P, et al. MIRU-VNTR typing of drug-resistant tuberculosis isolates in Greece. Ther Adv Respir Dis 2011; 5: 229-36.

383 Bernard C, Brossier F, Sougakoff W, et al. A surge of MDR and XDR tuberculosis in France among patients born in the Former Soviet Union. Euro Surveill 2013; 18: 20555.

384 Kubica T, Rusch-Gerdes S, Niemann S. The Beijing genotype is emerging among multidrug-resistant Mycobacterium tuberculosi strains from Germany. Int J Tuberc Lung Dis 2004; 8: 1107-13.

385 Farnia P, Masjedi MR, Varahram M, et al. The recent-transmission of Mycobacterium tuberculosis strains among Iranian and Afghan relapse cases: a DNA-fingerprinting using RFLP and spoligotyping. BMC Infect Dis 2008; 8: 109 .

386 Stoffels K, Allix-Beguec C, Groenen G, et al. From multidrug- to extensively drug-resistant tuberculosis: upward trends as seen from a 15-year nationwide study. PloS one 2013; 8: e63128.

387 O'Neil J. Tackling drug-resistant infections globally: final report and recommendations. In: Resistance RoA, ed. London, 2016

388 Pai M, Palamountain KM. New tuberculosis technologies: challenges for retooling and scale-up. Int J Tuberc Lung Dis 2012; 16: $1281-90$.

389 Smith J, Buse K, Gordon C. Civil society: the catalyst for ensuring health in the age of sustainable development. Global Health 2016; 12: 40 .

390 Jacovella D, Evans TG, Claeson M, Kagia R, Pablos-Mendez A. Global Financing Facility: where will the funds come from? Lancet 2016; 387: 121-12.

391 Yamey G, Batson A, Kilmarx PH, Yotebieng M. Funding innovation in neglected diseases. BMJ 2018; 360: k1182. 
392 https://www.usaid.gov/sites/default/files/documents/1864/ USAID_Global_Accelerator_Final_508.pdf (accessed Feb 24, 2019).

393 Gwatkin DR, Ergo A. Universal health coverage: friend or foe of health equity? Lancet 2011; 377: 2160-61.

394 Jamison DT, Alwan A, Mock CN, et al. Universal health coverage and intersectoral action for health: key messages from Disease Control Priorities, 3rd edition. Lancet 2018; 391: 1108-20.

395 Stenberg K, Hanssen O, Edejer TT, et al. Financing transformative health systems towards achievement of the health Sustainable Development Goals: a model for projected resource needs in 67 low-income and middle-income countries. Lancet Glob Health 2017; 5: e875-87.

396 Participants at the Bellagio Workshop on Implementing Pro-Poor Universal Health Coverage, Bump J, Cashin C, et al. Implementing pro-poor universal health coverage. Lancet Glob Health 2016; 4: e14-16.

397 WHO. Implementing the end TB strategy: the essentials. Geneva: World Health Organization, 2016.

398 Sismanidis C, Shete PB, Lienhardt C, Floyd K, Raviglione M. Harnessing the power of data to guide local action and end tuberculosis. J Infect Dis 2017; 216 (suppl 7): S669-72.

399 Reich MR, Harris J, Ikegami N, et al. Moving towards universal health coverage: lessons from 11 country studies. Lancet 2016; 387: 811-16

400 Devereux S, Sabates-Wheeler R. Transformative social protection. Working paper number 232. Institute for Development Studies. Brighton: University of Sussex; 2004.

401 Lonnroth K, Castro KG, Chakaya JM, et al. Tuberculosis control and elimination 2010-50: cure, care, and social development. Lancet 2010; 375: 1814-29.

402 Raviglione M, Zumla A, Marais B, Horton R, Motsoaledi A. A sustainable agenda for tuberculosis control and research. Lancet 2012; 379: 1077-78.

403 Lonnroth K, Raviglione M. Global epidemiology of tuberculosis: prospects for control. Semin Respir Crit Care Med 2008; 29: 481-91.

404 WHO. Closing the gapin a generation: health equity through action on the social determinants of health. Geneva: World Health Organization, 2008.

405 Carter DJ, Glaziou P, Lonnroth K, et al. The impact of social protection and poverty elimination on global tuberculosis incidence: a statistical modelling analysis of Sustainable Development Goal 1. Lancet Glob Health 2018; 6: e514-22.

406 Burki TK. The global cost of tuberculosis. Lancet Respir Med 2018; 6: 13 .

407 Comprehensive tuberculosis elimination plan act. Department of Health, Republic of the Philippines; July 27, 2015.

408 Ley de Prevención y Control de la Tuberculosis en el Perú. El Peruano, 2015

409 Oxlade O, Murray M. Tuberculosis and poverty: why are the poor at greater risk in India? PLoS One 2012; 7: e47533.

410 Bates MN, Khalakdina A, Pai M, Chang L, Lessa F, Smith KR. Risk of tuberculosis from exposure to tobacco smoke: a systematic review and meta-analysis. Arch Intern Med 2007; 167: 335-42.

411 Lai TC, Chiang CY, Wu CF, et al. Ambient air pollution and risk of tuberculosis: a cohort study. Occup Environ Med 2016; 73: 56-61.

412 Beaglehole R, Bonita R. Global public health: a scorecard. Lancet 2008; 372: 1988-96.

413 DeLuca A, Lessem E, Wegener D, Mingote LR, Frick M Von Delft D. The evolving role of advocacy in tuberculosis. Lancet Respir Med 2014; 2: 258-59.

414 Macq J, Torfoss T, Getahun H. Patient empowerment in tuberculosis control: reflecting on past documented experiences. Trop Med Int Health 2007; 12: 873-85.

415 Frick M, Henry I, Lessem E. Falling short of the rights to health and scientific progress: inadequate TB drug research and access. Health Hum Rights 2016; 18: 9-24.
416 London L, Cox H, Coomans F. Multidrug-resistant TB: implementing the right to health through the right to enjoy the benefits of scientific progress. Health Hum Rights 2016; 18: 25-41.

417 Craig GM, Daftary A, Engel N, O’Driscoll S, Ioannaki A. Tuberculosis stigma as a social determinant of health: a systematic mapping review of research in low incidence countries. Int J Infect Dis 2016; 56: 90-100.

418 Macintyre K, Bakker MI, Bergson S, et al. Defining the research agenda to measure and reduce tuberculosis stigmas. Int J Tuberc Lung Dis 2017; 21: 87-96.

419 Croft RP, Croft RA. Knowledge, attitude and practice regarding leprosy and tuberculosis in Bangladesh. Leprosy Rev 1999; 70: 34-42.

420 Wu PS, Chou P, Chang NT, Sun WJ, Kuo HS. Assessment of changes in knowledge and stigmatization following tuberculosis training workshops in Taiwan. J Formos Med Assoc 2009; 108: $377-85$.

421 Sommerland N, Wouters E, Mitchell EMH, et al. Evidence-based interventions to reduce tuberculosis stigma: a systematic review. Int J Tuberc Lung Dis 2017; 21: 81-86.

422 McGinty E, Pescosolido B, Kennedy-Hendricks A, Barry CL. Communication strategies to counter stigma and improve mental illness and substance use disorder policy. Psychiatr Serv 2017; 69: 136-46.

423 Wingfield T, Boccia D, Tovar MA, et al. Designing and implementing a socioeconomic intervention to enhance TB control: operational evidence from the CRESIPT project in Peru. BMC Public Health 2015; 15: 810.

424 Torrens AW, Rasella D, Boccia D, et al. Effectiveness of a conditional cash transfer programme on TB cure rate: a retrospective cohort study in Brazil. Trans $R$ Soc Trop Med Hyg 2016; 110: 199-206.

425 Sermrittirong S, Van Brakel WH, Bunbers-Aelen JF. How to reduce stigma in leprosy-a systematic literature review. Leprosy Rev 2014; 85: $149-57$.

426 Chikovore J, Hart G, Kumwenda M, Chipungu G, Desmond N, Corbett EL. TB and HIV stigma compounded by threatened masculinity: implications for TB health-care seeking in Malawi. Int J Tuberc Lung Dis 2017; 21: 26-33.

427 Ghebreyesus TA. All roads lead to universal health coverage. Lancet Glob Health 2017; 5: e839-40.

428 Horton R. Offline: UHC-one promise and two misunderstandings. Lancet 2018; 391: 1342.

429 Stop TB Partnership. Key targets and commitments. 2018. http://www.stoptb.org/global/advocacy/unhlm_targets.asp (accessed Dec 12, 2018).

430 WHO. The Abuja declaration: ten years on. 2018. https://www.who. int/healthsystems/publications/abuja_declaration/en/ (accessed Jan 31, 2019).

431 Interanational Labour Organization. World social protection report. 2017. https://www.social-protection.org/gimi/gess/RessourcePDF. action? ressource.ressourceId=54887 (accessed Jan 31, 2019).

432 WHO. TB control country profiles. 2017. https://www.who.int/ tobacco/surveillance/policy/country_profile/en/ (accessed Jan 31, 2019).

433 WHO. Global health observatory data. 2017. http://www.who.int/ gho/phe/en/ (accessed Jan 31, 2019).

434 Nabil H, Birdsall N, Kharas H. The Quality of Official Development Assistance (QuODA), third edition. 2014. https://www.cgdev.org/ sites/default/files/quality-official-development-assistance-quoda.pdf (accessed Feb 23, 2019).

(C) 2019. World Health Organization. Published by Elsevier Ltd/Inc/BV. All rights reserved. 